\title{
16. COMPOSITION, ALTERATION, AND ORIGIN OF THE BASEMENT LAVAS AND VOLCANICLASTIC ROCKS AT SITE 738, SOUTHERN KERGUELEN PLATEAU ${ }^{1}$
}

\author{
K. W. Mehl, ${ }^{2}$ P. R. Bitschene, ${ }^{2}$ H.-U. Schmincke, ${ }^{2}$ and J. Hertogen ${ }^{3}$
}

\begin{abstract}
During ODP Leg 119 one basement hole was drilled at Site 738, on the Southern Kerguelen Plateau. The $38.2 \mathrm{~m}$ of basement rocks drilled comprises three basaltic aa-lava flows with basal and top breccias, overlain by Turonian marine carbonates. Site 738 basalts probably erupted near a fracture zone, and were emplaced during the plateau-forming stage of Kerguelen Plateau evolution under quiet, subaerial to shallow water conditions.

The basalts are T-MORB, chemically resembling Mesozoic continental flood basalts of the southem hemisphere. Two slightly different magma batches are distinguished by $\mathrm{Fe}, \mathrm{Ti}, \mathrm{Al}, \mathrm{Zr}$, and $\mathrm{REE}$ concentrations. Prior to eruption, the magmas had undergone significant olivine and some clinopyroxene fractionation. Incompatible and immobile trace element concentrations and ratios point to a veined upper mantle source, where a refractory mineral assemblage retains $\mathrm{Nb}, \mathrm{Ta}$, and the HREE. The basaltic melts derived from this regionally veined, enriched upper mantle have high LREE, and especially $\mathrm{Ba}$ and Th concentrations and bear the DUPAL isotopic signature gained from deep- seated, recycled, old oceanic(?) crust.

A saponite-celadonite secondary mineral assemblage confines the alteration temperature to $<170^{\circ} \mathrm{C}$. Alteration is accompanied by net gains of $\mathrm{H}_{2} \mathrm{O}, \mathrm{CO}_{2}, \mathrm{~K}_{2} \mathrm{O}$, and $\mathrm{Rb}$, higher oxidation, minor $\mathrm{Na}_{2} \mathrm{O}, \mathrm{SiO}_{2}$ gains, and losses of $\mathrm{V}$ and $\mathrm{CaO}$. Released $\mathrm{Ca}$, together with $\mathrm{Ca}$ from seawater, precipitated as calcite in veins and vesicles, plumbed the circulation system and terminated the rock/open seawater interaction.
\end{abstract}

\section{INTRODUCTION}

The Kerguelen Plateau $\left(46^{\circ}-64^{\circ} \mathrm{S}\right)$ in the southern Indian Ocean is $2500 \mathrm{~km}$ long, $600 \mathrm{~km}$ wide, and rises $2-4 \mathrm{~km}$ above the surrounding ocean floor (Fig. 1). Two Ocean Drilling Program (ODP) legs (119 and 120) were designed to drill a latitudinal transect from Kerguelen Island $\left(49^{\circ} \mathrm{S}\right)$ to Prydz Bay $\left(68^{\circ} \mathrm{S}\right)$. Major objectives were to study the composition, structure, and age of the Kerguelen basement.

Site $738\left(62^{\circ} 42.55^{\prime} \mathrm{S}, 82^{\circ} 47.25^{\prime} \mathrm{E}\right)$ lies at the southernmost tip of the Kerguelen Plateau and drilling penetrated $486 \mathrm{~m}$ of mostly calcareous, Quaternary through lower Turonian sediments in $2263 \mathrm{~m}$ of water. Basement drilling was terminated at $533.8 \mathrm{~m}$ below seafloor (mbsf). Core recovery of the basement was $27.2 \mathrm{~m}$ or $71 \%$.

The magmatic and volcano-tectonic evolution of the Kerguelen archipelago and the Kerguelen Plateau has recently been outlined by Storey et al. (1988, and literature therein), Bassias et al. (1987), Davies et al. (1989), Schlich, Wise, et al. (1989), and Weis et al. (1989). The following components are considered to contribute to the formation of Kerguelen Plateau basaltic magmas:

1. A depleted, normal $(\mathrm{N})$ mid-ocean ridge basalt (MORB) reservoir.

2. A plume $(\mathrm{P})$ type $\mathrm{MORB}$ and ocean island basalt $(\mathrm{OIB})$ reservoir from an enriched, deep mantle source.

3. A highly ${ }^{87} \mathrm{Sr}_{\text {rad- }},{ }^{208} \mathrm{~Pb}_{\text {rad- }}$, and ${ }^{207} \mathrm{~Pb}_{\text {rad-enriched component }}$ derived either from subducted oceanic crust or from old continental lithosphere entrained in the Kerguelen Plateau. This component comes along with the P-MORB type reservoir.

${ }^{1}$ Barron, J., Larsen, B., et al., 1991. Proc. ODP, Sci. Results, 119: College Station, TX (Ocean Drilling Program).

${ }^{2}$ Institut für Mineralogie, Ruhr-Universităt, Postfach 102148, D-4630 Bochum, Federal Republic of Germany.

${ }^{3}$ Katholieke Universiteit, Leuven, Belgium.
The latter component is responsible for the $\mathrm{Sr}$ and $\mathrm{Pb}$ isotopic signature of all Kerguelen Plateau magmatic rocks (DUPAL anomaly; Hart, 1984). High Sr- and $\mathrm{Pb}$-isotope ratios (Alibert, this volume) confirm the DUPAL signature of Site 738 basalts. No indication of continental crustal rocks has been found in the basaltic rocks or in the overlying sedimentary sequence. This component, although never confirmed, is still not dismissed when discussing the origin of Kerguelen Plateau basalts (e.g., Davies et al., 1989).

The volcano-tectonic evolution of the southern Kerguelen Plateau (Bitschene et al., 1989) involves:

1. Quiet, subaerial to shallow-water tholeiitic (flood?) basalt volcanism and early rifting (Early to Middle Cretaceous plateau stage);

2. Emplacement, alteration, and erosion of ocean island-type alkali basalt volcanism, major rifting, and foundering (Late Cretaceous seamount stage);

3. Cessation of tectonic and magmatic activity and ongoing subsidence with fully marine conditions since the Paleocene (consolidation and submergence stage); shift of magmatic and tectonic activity toward the northern Kerguelen Plateau.

Site 738 magmatic activity belongs to the plateau-forming stage of Kerguelen Plateau evolution as evidenced by its pre-Turonian age and tholeiitic character.

\section{METHODS}

From fifty-five thin sections studied microscopically, twelve were analyzed by electron microprobe (EMP), using an automated wavelength dispersive system (Cameca). Operating conditions were $15 \mathrm{kV}$ accelerating voltage, 14 and $8 \mathrm{nA}$ beam current for primary and secondary minerals, respectively, and $20 \mathrm{~s}$ counting time. Beam diameter was about $8 \mu \mathrm{m}$. $\mathrm{Na}$ and $\mathrm{K}$ were measured first to minimize loss due to volatilization.

Bulk-rock chemical analyses were carried out by X-ray-fluorescence methods (XRF) on glass fusion beads, using an automated Philips PW 1400 spectrometer. The fusion beads consist of rock 


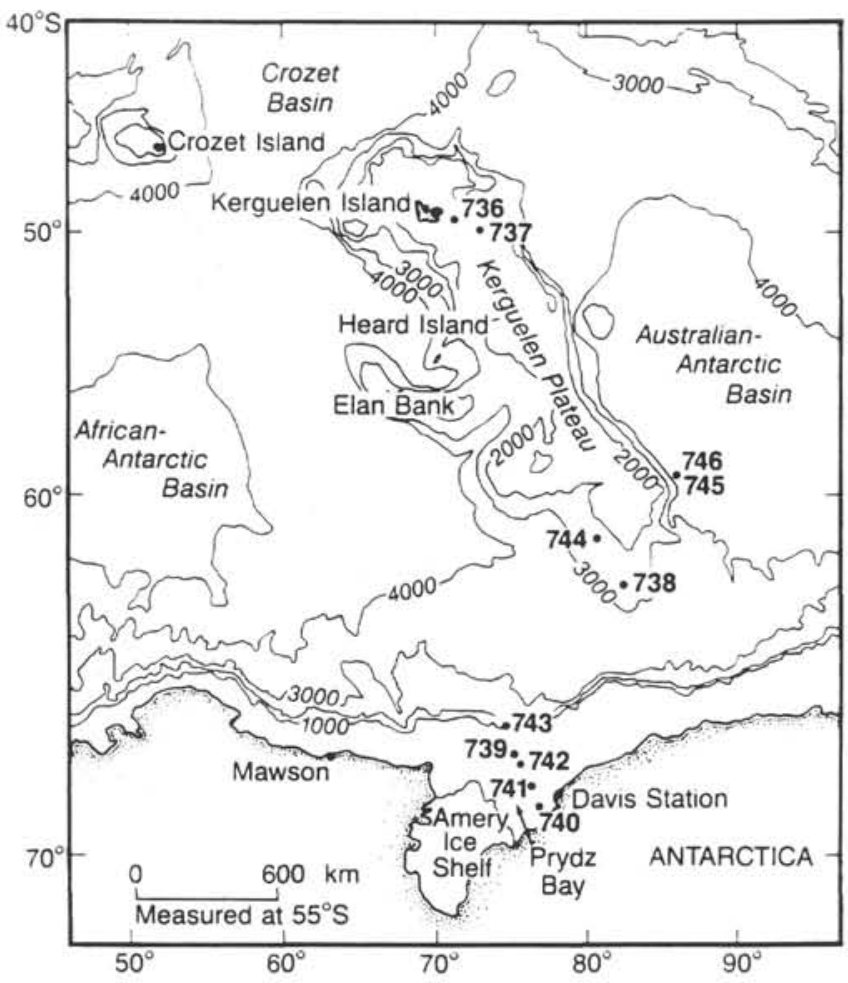

Figure 1. Bathymetric map of Kerguelen Plateau and Prydz Bay with Leg 119 drill sites. Contours in meters. Site 738 basement is studied in this report (from Barron, Larsen, et al., 1989).

powders, dried at $110^{\circ} \mathrm{C}$ for $24 \mathrm{hr}$, and flux (lithium metaborate and dilithium tetraborate; Spectromelt, Merck AR) in the ratio 1:4 melted at $1000^{\circ} \mathrm{C}$ for $20 \mathrm{~min}$ and poured into a $34-\mathrm{mm}$ - diameter pellet mold. Precision of the XRF major and trace element data is always better than $3 \%$, except for low $\mathrm{Nb}(<5 \mathrm{ppm})$ and $\mathrm{Rb}(<10 \mathrm{ppm})$ concentrations, which are near the detection level.

$\mathrm{Fe}^{2+}$ was determined by semiautomatic potentiometric titration of the hydrofluoric acid-silver perchlorate digested sample, with standard potassium bromide solution.

$\mathrm{CO}_{2}$ was determined by closed-system coulometric titration of barium perchlorate solution, into which the gases were led produced by heating the sample in a tube furnace at $1280^{\circ} \mathrm{C}$.

$\mathrm{H}_{2} \mathrm{O}^{+}$was measured by closed-system coulometric titration of a nonaqueous Karl Fischer reagent into which the carrier gas $\left(\mathrm{N}_{2}\right)$ was passed, containing water stripped from the sample by heating in a $\mathrm{Pt}$ crucible to $1300^{\circ} \mathrm{C}$ with an induction furnace.

Sample 119-738C-32R-CC, 12-14 cm, was treated with acetic acid for removal of carbonate before whole-rock analysis were carried out.

Only the basaltic clasts were analyzed from Sample 119-738C$32 \mathrm{R}-\mathrm{CC}, 0-4 \mathrm{~cm}$, after being handpicked under a binocular microscope.

Selected trace and rare earth elements (REE) of the freshest wholerock samples were analyzed by instrumental neutron activation analysis (INAA). The analytical methods were described in detail by Gijbels (1980) and Hertogen and Gijbels (1981).

\section{RESULTS}

\section{Lithology and Petrography}

Basaltic rocks are present in lithostratigraphic Unit VII and make up Unit VIII (Barron, Larsen, et al., 1989). In Subunit VII $(0.17 \mathrm{~m}$ recovery) subangular to rounded basaltic clasts $(0.5->20 \mathrm{~mm})$ are embedded in a bioclastic shallow-water limestone. Subunit VIIb $(0.05$ $m$ recovery) comprises a fractured and highly vesicular basalt overlain by a micritic limestone containing angular clasts of the underlying basalt. Large vesicles (about $20 \%$, up to $>10 \mathrm{~mm}$ long) are rimmed by brown and green sheet silicates and are filled with two generations of carbonate and minor "waxy mass." Fractures are filled by sparry calcite.

The basement (Unit VIII) consists of three massive basalt layers with intercalated breccias (Fig. 2). The lowermost basalt layer ( $>6.3$ $\mathrm{m}$ thick) is capped by about $3 \mathrm{~m}$ of breccia. The lower half of this breccia is dominated by tachylitic clasts in a dull red basaltic matrix, the upper half by basaltic clasts in a gray matrix. The middle basalt $(3.15 \mathrm{~m})$ shows vesicles $(3-10 \mathrm{~mm}$ in diameter; $<15 \%$ by volume $)$ at the base and in the top $10 \mathrm{~cm}$. The overlying breccia $(2.85 \mathrm{~m})$ comprises mostly vesicular, tachylitic clasts in a red matrix with 0.5 $\mathrm{m}$ of massive basalt intercalated in the lower part. The uppermost almost 10 -m-thick basalt layer is highly fractured. The brecciated top $(1.8 \mathrm{~m})$ grades into the overlying breccia $(3.5 \mathrm{~m})$. Mainly basaltic clasts lie in a dull red matrix in the lower $0.9 \mathrm{~m}$ of the breccia, whereas in the upper $2.6 \mathrm{~m}$ basaltic and tachylitic clasts are embedded in a red to dull red matrix.

The fine-grained basalts vary from grayish red brown to yellowish brown to almost black. They are massive to highly fractured. Narrow fractures are filled by green sheet silicates, and wider ones are rimmed by green sheet silicates and filled by sparry calcite. Vesicles $(0 \%-20 \%$, $0.5-5 \mathrm{~mm}$ in diameter, rarely exceeding $20 \mathrm{~mm}$ ) are filled by various secondary phases. Chilled margins are absent.

The massive basalts have subophitic to intergranular textures. Pegmatoid "schlieren" within the massive basalts (PI. 1) have dictytaxitic textures and comprise coarser crystals of plagioclase, clinopyroxene and ore minerals, and altered interstitial glass. Some "schlieren" contain geopetal vesicles filled by dark brown and blue green sheet silicates.

The basalts are aphyric to moderately plagioclase-phyric $(0.2-0.9$ $\mathrm{mm},<5 \%)$. Phenocrysts of clinopyroxene $(<0.5 \mathrm{~mm},<1 \%)$ and pseudomorphs of olivine $(<0.4 \mathrm{~mm},<1 \%)$ are area. $\mathrm{Fe} / \mathrm{Ti}$-oxides amounts

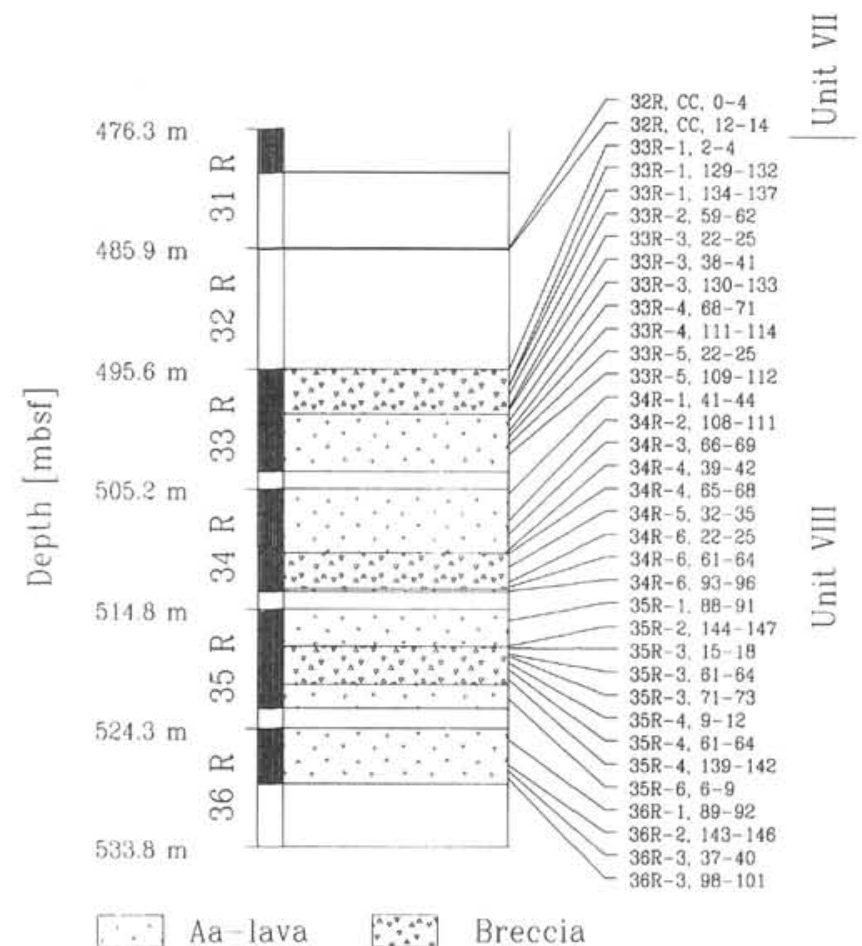

Figure 2. Stratigraphy of Site 738 basement and whole-rock samples studied. Recovery of cores indicated by dark bars. 
to $10 \%-20 \%(<0.4 \mathrm{~mm})$. The groundmass consists of plagioclase and clinophyroxene microlities in a dark mesostasis.

The breccias comprise mostly rounded to subangular, basaltic clasts $(0.5--80 \mathrm{~mm}, 40 \%-90 \%$ of the total breccia) embedded in a fine-grained, basaltic, highly altered, and varicolored matrix with red to dull red and black, partly green shades. The breccias are unstratified and neither fossils nor terrigenous detritus are mixed or interlayered with the basalt and breccia. Two main types of clasts were distinguished: (1) Tachylitic clasts are gray to brownish black with a tachylitic matrix (about 50\%) and a high vesicle content $(5 \%-20 \%$, mostly $1-2 \mathrm{~mm}$ in diameter); (2) basaltic clasts are greenish to brown and correspond to the massive basalts. The breccias do not represent hyaloclastic deposits but top breccia (tachylitic clasts) and base breccia (basaltic clasts).

\section{Chemistry}

\section{Primary Phases}

Electron microprobe analyses of plagioclase (Fig. 3 and Table 1 with selected analyses) show variable anorthite (An) content within single samples (e.g., Sample 119-738C-36R-2, 143-146 cm, with An48-73, microphenocrysts reaching An48-62, and phenocrysts An6273). Plagioclase compositions range from $\mathrm{An}_{69} \mathrm{Ab}_{30} \mathrm{Or}_{1}$ to $\mathrm{An}_{44} \mathrm{Ab}_{53} \mathrm{Or}_{3}$. Feldspars in two pegmatoid "schlieren" (PI. 1) show An61-67 (Sample 119-738C-36R-2, 143-146 cm) and An40-49 (Sample $119-738 \mathrm{C}-33 \mathrm{R}-5,30-32 \mathrm{~cm}$ ). A few plagioclase crystals in Subunit VIlb show oscillatory zoning (e.g., An68-An65-An78-An59 and An71-An74-An65). The amount of the albite and orthoclase component increases linearly with decreasing An (Fig. 3).

All analyzed pyroxenes are augitic (En32-51, Fs8-29, Wo23-35; Fig. 4 and Table 2 with selected analyses) except for two of pigeonites (Sample 119-738C- 36R-2, 143-146 cm: En 64-68, Fs19-24, Wo6-8, lowermost two quadrangles in Fig. 4). Some clinopyroxene phenocrysts have high $\mathrm{Cr}_{2} \mathrm{O}_{3}$ concentrations ( $>1 \%$, Table 2 ).

\section{Secondary Phases}

Secondary phases are dominantly brown and green sheet silicates apart from carbonate. Several phases were distinguished optically and by electron microprobe analysis (Table 3 and Fig. 5).

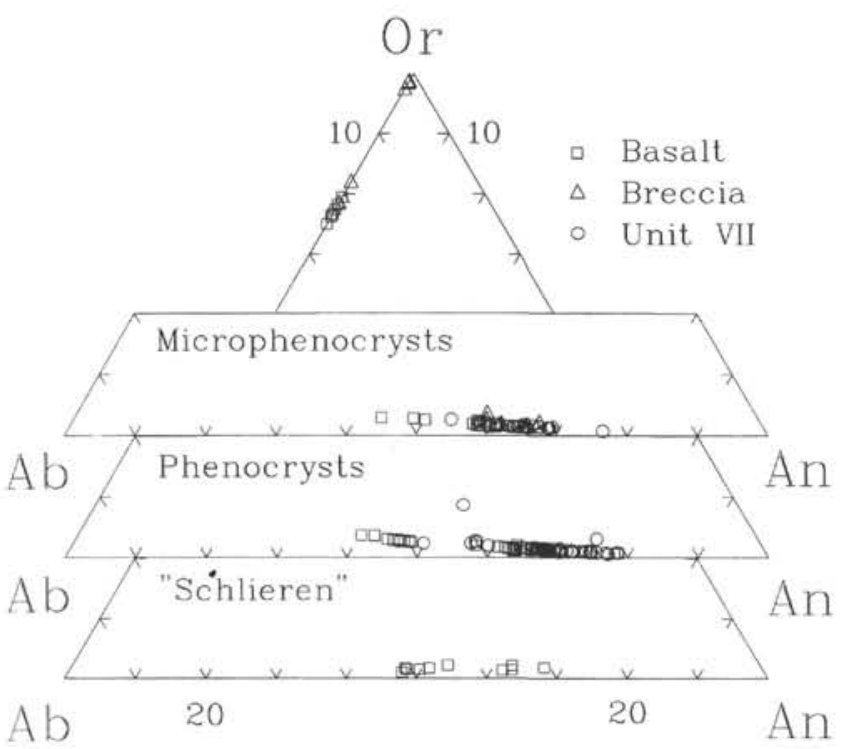

Figure 3. Compositional variation of plagioclase in "pegmatoid schlieren," as phenocrysts and microphenocrysts, and secondary K-feldspar replacing microphenocrysts and glass in the triangle orthoclase(Or)-albite(Ab)- anorthite(An).
Celadonite is microcrystalline to fibrous, some scaly crystals show yellow or grass green to bluish green pleochroism. Celadonite has replaced interstitial glass, plagioclase, and clinopyroxene, and occurs as a major filling of vesicles and veins. Electron microprobe analyses show high iron $(17 \%-25 \% \mathrm{FeO})$ and potassium $\left(3 \%-9.5 \% \quad \mathrm{~K}_{2} \mathrm{O}\right)$ concentrations. The analyses have more $\mathrm{SiO}_{2}$ and less $\mathrm{FeO}$ than those reported from Deep Sea Drilling Project (DSDP) Leg 37 (Andrews, 1980).

Green saponite is slightly pleochroic with green to olive green or greenish brown tones. The fibrous, rarely microcrystalline crystals are radially arranged in vesicle fillings and are up to $150 \mu \mathrm{m}$ long. Green saponites are $\mathrm{Mg}$-rich (18\%-20.5\% $\mathrm{MgO}$ ) and low in iron $(5.5 \%-7 \%$ $\mathrm{FeO}$ ) and correspond to the $\mathrm{Mg}$-rich, $\mathrm{Fe}$-poor saponite subgroup of Andrews (1980), but with significantly lower $\mathrm{SiO}_{2}$ values.

Colorless to coffee brown, fibrous to radially arranged, brown saponite replaces plagioclase and occurs as a minor vesicle filling. The brown saponites have lower $\mathrm{MgO}(9.5 \%-17.2 \%)$ and higher $\mathrm{FeO}$ $(9.8 \%-15.6 \%)$ concentrations compared to the green saponites and may correspond to the Fe- rich saponite subgroup of Andrews (1980).

Montmorillonite(?) occurs in two varieties. One variety of montmorillonite (A) is an almost colorless to light brown, nearly isotropic phase and occurs as angular grains in Subunit VIIb (Sample 119$738 \mathrm{C}-32 \mathrm{R}-\mathrm{CC}, 10-12 \mathrm{~cm}$ ). The refractive index is $<1.54$. The material is replaced along the rim and cracks by brown sheet silicates and, along cracks, by calcite.

Electron microprobe analyses show high silica $\left(54 \%-61 \% \mathrm{SiO}_{2}\right)$ and alumina $\left(17 \%-19.2 \% \mathrm{Al}_{2} \mathrm{O}_{3}\right)$ contents which fit well the mean composition of 101 montmorillonites-beidellites reported by Weaver and Pollard (1973).

The other montmorillonite(?) variety (B) is cryptocrystalline with various brown shades and concentrically rims and fills vesicles and veins. It was described in Barron, Larsen, et al. (1989) as a "waxy mass." Concentric at vesicle rims, it partly passes over to geopetal vesicle fillings. Some vesicles are completely filled by this phase that generally exhibits shrinkage cracks. Electron microprobe analyses show lower $\mathrm{Al}(11.5 \%-17.6 \%)$ and higher $\mathrm{Mg}(7.9 \%-10.3 \%)$ concentrations compared to the mean composition of 101 montmorillonitesbeidellites reported by Weaver and Pollard (1973).

Brown sheet silicate phases show various brown colors and are of varying crystallinity (microcrystalline to fibrous). They replace interstitial glass, glass shards, plagioclase and clinopyroxene, line basaltic clasts (Sample 119-738C-32R-CC, 10-12 cm) and phenocrysts in a pegmatoid "schliere" (Sample 119- 738C-34R-4, 39-42), and occur in pseudomorphs after olivine, and as concentric rims and fillings of vesicles and veins.

Calcite replaces plagioclase (especially in the core and along cracks) and interstitial glass. Blocky calcite is a major vesicle- and vein-filling mineral and also occurs in diktytaxitic pegmatoid "schlieren" (e.g., Sample 119-738C-34R-4, 39-42 cm). Vesicle-filling palisade calcite cementation occurs only in Subunit VIIb. Electron microprobe analyses reveal almost pure $\mathrm{Ca}$ carbonate. $\mathrm{MgO}$ is low $(0.3 \%-1.2 \%)$, whereas $\mathrm{P}_{2} \mathrm{O}_{5}$ concentrations $(0.8 \%-1.0 \%)$ are high and reliable, but cannot readily be explained. Microcrystalline apatite fibers or other phosphate inclusions are suspected to be the reason for the unusual $\mathrm{P}$ contents in the calcite. Late stage vesicle- filling calcite contains significant $\mathrm{MnO}(2.9 \%-3.6 \%)$.

A colorless $\mathrm{SiO}_{2}$ phase with weak birefringence occurs as round aggregates in vesicles in only one thin section (Sample 119-738C$36 \mathrm{R}-1,148-150 \mathrm{~cm}$ ) and is thought to be chalcedony. Metasomatic $\mathrm{SiO}_{2}$ replaces carbonate in Subunit VIIa.

Dirty brown, blocky K-feldspar crystals with weak birefringence replace former interstitial glass and also occur in pegmatoid "schlieren," there replacing glassy matrix. Electron microprobe analyses reveal Or75-82 (Table 3) which attributes them a magmatic origin. Microphenocrysts in tachylitic clasts have Or98-99.

Pseudomorphs after olivine also occur in two types: 
Table 1. EMP analyses of primary and secondary feldspar of selected Hole 738C samples. Calculations based on $\mathrm{O}=32$. Remarks: $1=$ microphenocryst, 2 = phenocryst, 3 = in "pegmatoid schliere," 4 = replacing microphenocryst or glass.

\begin{tabular}{|c|c|c|c|c|c|c|c|c|c|c|c|c|c|c|c|}
\hline $\begin{array}{c}\text { Corc, } \\
\text { Section, } \\
\text { Interval } \\
{[\mathrm{cm}]}\end{array}$ & $\begin{array}{c}33 R-3 \\
18-120\end{array}$ & $\begin{array}{l}33 R-5 \\
30-32\end{array}$ & $\begin{array}{l}33 R-5 \\
30-32\end{array}$ & $\begin{array}{l}33 R-5 \\
30-32\end{array}$ & $\begin{array}{l}33 R-5 \\
30-32\end{array}$ & $\begin{array}{l}33 \mathrm{R}-5 \\
30-32\end{array}$ & $\begin{array}{l}33 R-5 \\
30-32\end{array}$ & $\begin{array}{l}34 R-4 \\
39-42\end{array}$ & $\begin{array}{l}34 \mathrm{R}-4 \\
39-42\end{array}$ & $\begin{array}{l}34 \mathrm{R}-4 \\
39-42\end{array}$ & $\begin{array}{l}34 \mathrm{R}-4 \\
39-42\end{array}$ & $\begin{array}{l}34 R-4 \\
39-42\end{array}$ & $\begin{array}{l}34 R-5 \\
44-46\end{array}$ & $\begin{array}{l}34 R-5 \\
44-46\end{array}$ & $\begin{array}{l}34 R-5 \\
44-46\end{array}$ \\
\hline Remarks & 4 & 1 & 1 & 1 & 4 & 4 & 4 & 3 & 3 & 3 & 1 & 1 & 1 & 1 & 1 \\
\hline $\mathrm{SiO}_{2}$ & 63.94 & 53.56 & 55.14 & 53.37 & 64.54 & 64.85 & 65.57 & 54.98 & 54.42 & 55.69 & 57.49 & 51.93 & 52.86 & 52.38 & 63.82 \\
\hline $\mathrm{TiO}_{2}$ & 1.03 & 0.09 & 0.11 & 0.07 & 0.57 & 0.72 & 0.29 & 0.08 & 0.09 & 0.09 & 0.11 & 0.06 & 0.07 & 0.09 & 0.03 \\
\hline $\mathrm{Al}_{2} \mathrm{O}_{3}$ & 17.89 & 28.51 & 27.13 & 28.22 & 17.46 & 17.67 & 18.16 & 27.40 & 27.74 & 27.20 & 26.00 & 29.42 & 29.12 & 29.47 & 18.52 \\
\hline $\mathrm{MnO}$ & 0.01 & 0.00 & 0.00 & 0.00 & 0.02 & 0.04 & 0.00 & 0.06 & 0.03 & 0.03 & 0.02 & 0.00 & 0.00 & 0.01 & 0.00 \\
\hline $\mathrm{MgO}$ & 0.00 & 0.05 & 0.06 & 0.09 & 0.30 & 0.10 & 0.03 & 0.08 & 0.22 & 0.10 & 0.10 & 0.23 & 0.23 & 0.24 & 0.05 \\
\hline $\mathrm{CaO}$ & 0.00 & 11.70 & 10.09 & 11.71 & 0.00 & 0.00 & 0.00 & 9.73 & 10.87 & 9.77 & 8.76 & 13.12 & 12.83 & 13.07 & 0.00 \\
\hline $\mathrm{Na}_{2} \mathrm{O}$ & 1.91 & 4.46 & 5.29 & 4.42 & 2.48 & 2.37 & 2.20 & 5.84 & 5.00 & 5.70 & 5.95 & 3.83 & 3.77 & 3.40 & 0.28 \\
\hline $\mathrm{K}_{2} \mathrm{O}$ & 13.29 & 0.28 & 0.45 & 0.36 & 12.10 & 12.89 & 12.93 & 0.18 & 0.37 & 0.27 & 0.50 & 0.20 & 0.28 & 0.37 & 16.44 \\
\hline $\mathrm{SrO}$ & nd & nd & nd & nd & nd & nd & nd & nd & nd & nd & nd & nd & nd & nd & nd \\
\hline $\mathrm{Fe}^{3+}$ & 0.00 & 0.00 & 0.00 & 0.00 & 0.00 & 0.00 & 0.00 & 0.12 & 0.03 & 0.03 & 0.00 & 0.00 & 0.00 & 0.00 & 0.03 \\
\hline $\mathrm{Fe}^{2+}$ & 0.15 & 0.15 & 0.15 & 0.15 & 0.20 & 0.11 & 0.08 & 0.00 & 0.09 & 0.09 & 0.13 & 0.11 & 0.11 & 0.10 & 0.00 \\
\hline Mn & 0.00 & 0.00 & 0.00 & 0.00 & 0.00 & 0.01 & 0.00 & 0.01 & 0.01 & 0.00 & 0.00 & 0.00 & 0.00 & 0.00 & 0.00 \\
\hline $\mathrm{Mg}$ & 0.00 & 0.01 & 0.02 & 0.02 & 0.08 & 0.03 & 0.01 & 0.02 & 0.06 & 0.03 & 0.03 & 0.06 & 0.06 & 0.07 & 0.01 \\
\hline $\mathrm{Ca}$ & 0.00 & 2.28 & 1.97 & 2.29 & 0.00 & 0.00 & 0.00 & 1.89 & 2.11 & 1.89 & 1.69 & 2.57 & 2.50 & 2.56 & 0.00 \\
\hline $\mathrm{Na}$ & 0.69 & 1.58 & 1.87 & 1.57 & 0.89 & 0.85 & 0.79 & 2.05 & 1.76 & 2.00 & 2.08 & 1.36 & 1.33 & 1.21 & 0.10 \\
\hline $\mathrm{K}$ & 3.16 & 0.07 & 0.10 & 0.08 & 2.87 & 3.04 & 3.04 & 0.04 & 0.09 & 0.06 & 0.12 & 0.05 & 0.06 & 0.09 & 3.90 \\
\hline Sr & nd & nd & nd & nd & nd & nd & nd & nd & nd & nd & nd & nd & nd & nd & nd \\
\hline $\operatorname{Sum} Z$ & 15.85 & 15.86 & 15.85 & 15.83 & 15.85 & 15.85 & 16.03 & 15.82 & 15.81 & 15.85 & 15.91 & 15.81 & 15.88 & 15.92 & 15.94 \\
\hline Sum $X$ & 3.85 & 3.92 & 3.94 & 3.94 & 3.77 & 3.89 & 3.83 & 3.98 & 3.96 & 3.95 & 3.89 & 3.97 & 3.90 & 3.85 & 4.01 \\
\hline Or & 82.06 & 1.66 & 2.64 & 2.11 & 76.26 & 78.18 & 79.42 & 1.04 & 2.19 & 1.57 & 2.98 & 1.15 & 1.66 & 2.26 & 97.45 \\
\hline $\mathrm{Ab}$ & 17.94 & 40.16 & 47.39 & 39.74 & 23.74 & 21.82 & 20.58 & 51.53 & 44.43 & 50.55 & 53.49 & 34.20 & 34.16 & 31.30 & 2.55 \\
\hline
\end{tabular}

Type I comprises irregularly oriented, fibrous, brown sheet silicates with a slightly anisotropic, colorless, microcrystalline mineral (probably a $\mathrm{SiO}_{2}$ phase) near the center. An irregular cleavage is characteristic. Anhedral opaque minerals may be embedded in the center and/or they are accumulated at the rim.

Type II is colorless to faintly greenish pleochroic to dark orange, the latter being caused by an increasing amount of $\mathrm{Fe}$ (up to $>50 \%$ $\mathrm{FeO}$; cf. Table 3 ). The orange variety differs from iddingsite (Deer et al., 1982) by significant lower $\mathrm{MgO}$ concentrations. The colorless variety has significant iron $(10.3 \%-18 \% \mathrm{FeO})$ and relatively low silicon $\left(40.2 \%-48.7 \% \mathrm{SiO}_{2}\right)$ contents. Most pseudomorphs are strongly "cleaved" and show almost parallel extinction and high birefringence and may correspond to type IV "iddingsite" of Baker and Haggerty (1967).

The compositions of typical vesicle filling phases (Fig. 6) were analyzed by electron microprobe from rim to core for two parageneses (Fig. 7). Paragenesis B ("waxy mass"-light brown sheet mineral-green saponite-calcite) shows increasing concentrations of $\mathrm{FeO}$ and $\mathrm{MgO}$ and decreasing $\mathrm{K}_{2} \mathrm{O}, \mathrm{Na}_{2} \mathrm{O}, \mathrm{Al}_{2} \mathrm{O}_{3}$, and $\mathrm{SiO}_{2}$. The pattern of paragenesis A (varicolored sheet silicates, see Figs. 6A and 7) is dominated by the precipitation of celadonite (= bright green) with a maximum of $\mathrm{K}_{2} \mathrm{O}$ and minimum of $\mathrm{CaO}$ and $\mathrm{MgO}$. $\mathrm{CaO}$ and $\mathrm{Na}_{2} \mathrm{O}$ generally decrease from rim to core whereas $\mathrm{FeO}$ shows ambiguous behavior.

\section{Bulk Rock}

All values, graphs, and interpretations of bulk rock chemistry (Table 4, Figs. 8, 9, 10) are based on normalized, volatile-free calcu- lations, except for the Rare Earth Elements (REE, Fig. 11). The microscopically and chemically freshest basalt samples (119-738C$34 \mathrm{R}-2,108-111 \mathrm{~cm} ; 119-738 \mathrm{C}-36 \mathrm{R}-2,143-146 \mathrm{~cm} ; 119-738 \mathrm{C}-36 \mathrm{R}-$ $3,37-40 \mathrm{~cm} ; 119-738 \mathrm{C}-36 \mathrm{R}-3,98-101 \mathrm{~cm}$ ) have the lowest $\mathrm{H}_{2} \mathrm{O}$ $(0.75 \%-1.32 \%), \mathrm{CO}_{2}(0.06 \%-0.12 \%)$, and $\mathrm{Fe}_{2} \mathrm{O}_{3} / \mathrm{FeO}(0.45 \%-$ $0.59 \%)$, and low $\mathrm{K}_{2} \mathrm{O}(0.38 \%-1.10 \%)$ and $\mathrm{Rb}(5-27 \mathrm{ppm})$ concentrations. Their $\mathrm{K} / \mathrm{Rb}$ ratios lie between 166 and 307, whereas altered samples have much higher K/Rb ratios (e.g., Sample 119-738C-34R$6,61-64 \mathrm{~cm}: 2550)$. The fresh samples are used when discussing source characteristics of the basaltic suite. Ratios of alteration-insensitive elements $(\mathrm{Zr}, \mathrm{Nb}, \mathrm{Ta}, \mathrm{Ti}, \mathrm{REE}, \mathrm{Cr}$ ) from all samples further constrain the source region and, together with normalized absolute concentrations of alteration-insensitive elements, help to explain small intraflow and intrasuite variations.

\section{Classification}

On a normative basis, the freshest samples are olivine-free, hypersthene- normative quartz-tholeiites with high normative orthoclase and albite (Tables 1 and 5). The tholeiitic character is substantiated by the presence of $\mathrm{Mg}$-rich and $\mathrm{Mg}$-poor clinopyroxene (Table 4). The freshest samples also plot into the tholeiitic field within $\mathrm{a}_{2} \mathrm{O}+\mathrm{Na}_{2} \mathrm{O}$ vs. $\mathrm{SiO}_{2}$ diagram (e.g., MacDonald and Katsura, 1964). High $\mathrm{Fe}^{+++}$, $\mathrm{Ca}$ loss, and $\mathrm{K}$ gain of the altered rocks do not allow proper norm calculations or TAS plots of all basement samples. But all samples are considered to be originally quartz-tholeiitic basalts on grounds of the close relationship in incompatible and immobile element concentrations, and the spatial coherence. 
Table 1. (continued).

\begin{tabular}{|c|c|c|c|c|c|c|c|c|c|c|c|c|c|c|}
\hline $\begin{array}{c}\text { Core, } \\
\text { Section, } \\
\text { Interval } \\
{[\mathrm{cm}]}\end{array}$ & $\begin{array}{l}34 R-5 \\
44-46\end{array}$ & $\begin{array}{l}34 R-5 \\
44-46\end{array}$ & $\begin{array}{l}35 \mathrm{R}-3 \\
15-18\end{array}$ & $\begin{array}{c}36 R-1 \\
48-150\end{array}$ & $\begin{array}{c}36 R-1 \\
48-150\end{array}$ & $\begin{array}{c}36 R-1 \\
48-150\end{array}$ & $\begin{array}{c}36 R-2 \\
43-146\end{array}$ & $\begin{array}{c}36 R-2 \\
43-146\end{array}$ & $\begin{array}{c}36 R-2 \\
43-146\end{array}$ & $\begin{array}{c}36 R-2 \\
43-146\end{array}$ & $\begin{array}{c}36 R-2 \\
43-146\end{array}$ & $\begin{array}{c}36 R-2 \\
43-146\end{array}$ & $\begin{array}{c}36 R-2 \\
43-146\end{array}$ & $\begin{array}{c}36 R-2 \\
43-146\end{array}$ \\
\hline Remarks & 1 & 1 & 1 & 2 & 2 & 2 & 3 & 3 & 2 & 2 & 2 & 1 & 1 & 1 \\
\hline $\mathrm{SiO}_{2}$ & 52.68 & 64.38 & 54.08 & 52.05 & 52.37 & 51.07 & 52.71 & 51.78 & 53.79 & 51.11 & 52.58 & 55.29 & 52.60 & 53.87 \\
\hline $\mathrm{TiO}_{2}$ & 0.05 & 0.01 & 0.09 & 0.06 & 0.07 & 0.05 & 0.04 & 0.12 & 0.13 & 0.00 & 0.06 & 0.11 & 0.21 & 0.03 \\
\hline $\mathrm{Al}_{2} \mathrm{O}_{3}$ & 28.97 & 18.53 & 27.86 & 29.07 & 29.22 & 30.29 & 28.03 & 22.14 & 27.53 & 29.16 & 28.03 & 26.15 & 27.99 & 27.63 \\
\hline $\mathrm{Fe}_{2} \mathrm{O}_{3}$ & 0.00 & 0.22 & 0.00 & 0.43 & 0.11 & 0.00 & 1.06 & 4.26 & 0.82 & 0.58 & 0.56 & 0.90 & 0.10 & 0.66 \\
\hline $\mathrm{FeO}$ & 0.69 & 0.00 & 0.98 & 0.39 & 0.73 & 0.50 & 0.00 & 0.00 & 0.00 & 0.01 & 0.00 & 0.00 & 0.64 & 0.37 \\
\hline $\mathrm{MnO}$ & 0.02 & 0.01 & 0.00 & 0.01 & 0.04 & 0.03 & 0.00 & 0.10 & 0.05 & 0.02 & 0.06 & 0.04 & 0.05 & 0.02 \\
\hline $\mathrm{MgO}$ & 0.24 & 0.02 & 0.15 & 0.11 & 0.09 & 0.17 & 0.14 & 2.81 & 0.09 & 0.21 & 0.21 & 0.03 & 0.06 & 0.09 \\
\hline $\mathrm{CaO}$ & 12.83 & 0.00 & 11.50 & 12.91 & 12.91 & 13.90 & 12.80 & 13.37 & 12.02 & 14.26 & 12.88 & 10.00 & 12.71 & 11.91 \\
\hline $\mathrm{Na}_{2} \mathrm{O}$ & 3.71 & 0.14 & 4.18 & 4.06 & 4.03 & 3.43 & 4.26 & 3.39 & 4.71 & 3.40 & 4.13 & 5.61 & 4.08 & 4.51 \\
\hline $\mathrm{K}_{2} \mathrm{O}$ & 0.22 & 16.87 & 0.46 & 0.22 & 0.25 & 0.15 & 0.24 & 0.30 & 0.35 & 0.17 & 0.26 & 0.49 & 0.28 & 0.42 \\
\hline $\mathrm{SrO}$ & nd & nd & nd & nd & nd & nd & 0.29 & 0.32 & 0.25 & 0.30 & 0.27 & 0.29 & 0.38 & 0.32 \\
\hline Total & 99.40 & 100.19 & 99.30 & 99.32 & 99.82 & 99.58 & 99.57 & 98.59 & 99.74 & 99.22 & 99.04 & 98.91 & 99.10 & 99.83 \\
\hline $\mathrm{Si}$ & 9.64 & 11.90 & 9.92 & 9.52 & 9.53 & 9.33 & 9.65 & 9.69 & 9.80 & 9.42 & 9.67 & 10.11 & 9.69 & 9.83 \\
\hline $\mathrm{Ti}$ & 0.01 & 0.00 & 0.01 & 0.01 & 0.01 & 0.01 & 0.01 & 0.02 & 0.02 & 0.00 & 0.01 & 0.02 & 0.03 & 0.00 \\
\hline $\mathrm{Al}$ & 6.25 & 4.04 & 6.02 & 6.27 & 6.26 & 6.52 & 6.05 & 4.88 & 5.91 & 6.33 & 6.08 & 5.64 & 6.08 & 5.94 \\
\hline $\mathrm{Fe}^{3+}$ & 0.00 & 0.03 & 0.00 & 0.06 & 0.01 & 0.00 & 0.15 & 0.60 & 0.11 & 0.08 & 0.08 & 0.12 & 0.01 & 0.09 \\
\hline $\mathrm{Fe}^{2+}$ & 0.11 & 0.00 & 0.15 & 0.06 & 0.11 & 0.08 & 0.00 & 0.00 & 0.00 & 0.00 & 0.00 & 0.00 & 0.10 & 0.06 \\
\hline $\mathrm{Mn}$ & 0.00 & 0.00 & 0.00 & 0.00 & 0.01 & 0.00 & 0.00 & 0.02 & 0.01 & 0.00 & 0.01 & 0.01 & 0.01 & 0.00 \\
\hline $\mathrm{Mg}$ & 0.06 & 0.01 & 0.04 & 0.03 & 0.03 & 0.05 & 0.04 & 0.78 & 0.02 & 0.06 & 0.06 & 0.01 & 0.02 & 0.02 \\
\hline $\mathrm{Ca}$ & 2.52 & 0.00 & 2.26 & 2.53 & 2.52 & 2.72 & 2.51 & 2.68 & 2.35 & 2.82 & 2.54 & 1.96 & 2.51 & 2.33 \\
\hline $\mathrm{Na}$ & 1.31 & 0.05 & 1.49 & 1.44 & 1.42 & 1.21 & 1.51 & 1.23 & 1.66 & 1.22 & 1.47 & 1.99 & 1.46 & 1.60 \\
\hline K & 0.05 & 3.98 & 0.11 & 0.05 & 0.06 & 0.03 & 0.06 & 0.07 & 0.08 & 0.04 & 0.06 & 0.11 & 0.07 & 0.10 \\
\hline $\mathrm{Sr}$ & nd & nd & nd & nd & nd & nd & 0.03 & 0.03 & 0.03 & 0.03 & 0.03 & 0.03 & 0.04 & 0.03 \\
\hline Sum Z & 15.89 & 15.93 & 15.94 & 15.78 & 15.79 & 15.85 & 15.70 & 14.57 & 15.72 & 15.75 & 15.75 & 15.75 & 15.76 & 15.77 \\
\hline Sum $X$ & 3.88 & 4.03 & 3.85 & 4.02 & 4.00 & 3.97 & 4.08 & 3.98 & 4.09 & 4.07 & 4.07 & 4.06 & 4.03 & 4.02 \\
\hline Or & 1.31 & 98.76 & 2.79 & 1.29 & 1.43 & 0.86 & 1.37 & 1.80 & 1.99 & 0.98 & 1.50 & 2.81 & 1.63 & 2.43 \\
\hline $\mathrm{Ab}$ & 33.87 & 1.24 & 38.57 & 35.79 & 35.60 & 30.58 & 37.07 & 30.89 & 40.66 & 29.85 & 36.17 & 48.96 & 36.15 & 39.67 \\
\hline An & 64.82 & 0.00 & 58.64 & 62.91 & 62.97 & 68.56 & 61.55 & 67.32 & 57.35 & 69.17 & 62.33 & 48.23 & 62.22 & 57.90 \\
\hline
\end{tabular}

\section{Element Variation with Depth}

Some elements show considerable variations when basalts and volcaniclastics are considered (Fig. 8), e.g., $\mathrm{Cr}$ (51-237 ppm), Ba (119-517 ppm), Ni (24-82 ppm), Cu (23-173 ppm), Rb (5-47 ppm), and Y (24-58 ppm). Other elements have rather similar concentrations, e.g., $\mathrm{SiO}_{2}$ (47.07-53.34\%), $\mathrm{TiO}_{2}(1.42-2.27 \%)$, and $\mathrm{Zr}$ (128$197 \mathrm{ppm})$.

Cr (Fig. 8, Table 6) averages $94 \pm 43$ ppm for the samples below $497 \mathrm{mbsf}$, and then rises abruptly to $237 \mathrm{ppm}$ in the top basaltic flow and volcaniclastic unit at 495.5-497 mbsf. Ni decreases slightly with depth. The alkali metals $\mathrm{Rb}, \mathrm{K}$, and $\mathrm{Na}$, as well as $\mathrm{Al}$ are also roughly negatively correlated with depth. In general, the clastic and brecciated rocks are the most sensitive for alteration and display larger scatter in element concentrations than the basalts which dominate the section below $521 \mathrm{mbsf}$.

\section{Element Variation with $\mathrm{ZR}$ as Differentiation Index}

$\mathrm{Zr}$ is used as differentiation index (Fig. 9) as alteration may have led to variations in $\mathrm{MgO}$ unrelated to differentiation processes. The basalt flow samples (quadrangles in Fig. 9) show positive correlations of $\mathrm{TiO}_{2}, \mathrm{Al}_{2} \mathrm{O}_{3}, \mathrm{Na}_{2} \mathrm{O}, \mathrm{V}$, and $\mathrm{Sr}$ with $\mathrm{Zr}$. $\mathrm{Cr}, \mathrm{SiO}_{2}$, and $\mathrm{P}_{2} \mathrm{O}_{5}$, and, with a larger scatter, $\mathrm{Nb}, \mathrm{Ba}$, and $\mathrm{Ni}$ have rather constant levels at different $\mathrm{Zr}$ concentrations. All other (alteration sensitive) elements show a large scatter with $\mathrm{Zr}$, similar to the scatter shown by the breccias.

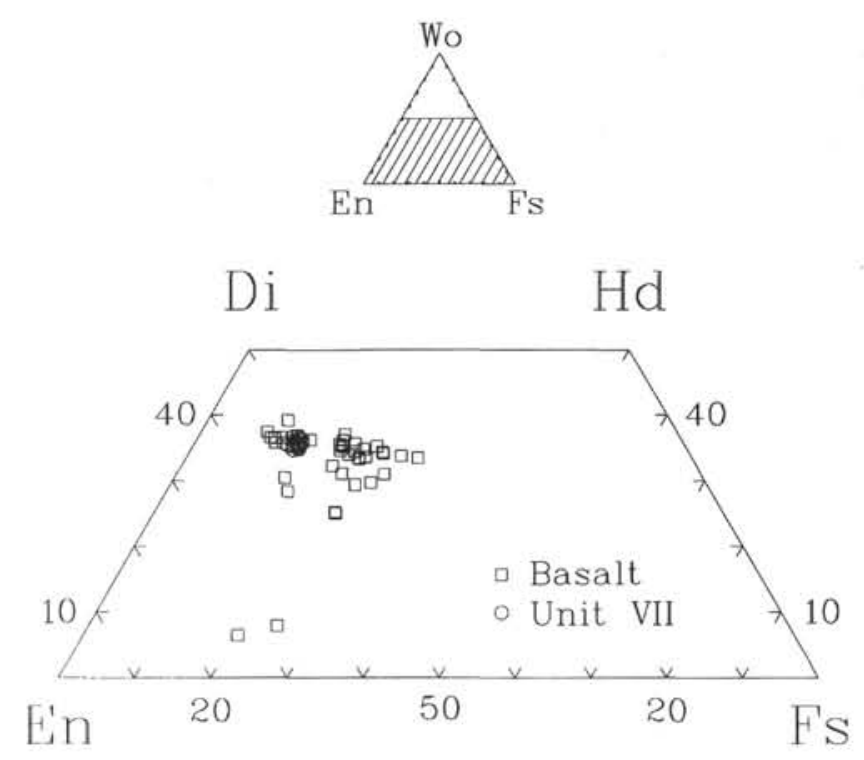

Figure 4. Chemical composition of clinopyroxenes in the quadrilateral enstatite(En)-ferrosilite(Fs)-hedenbergite(Hd)-diopside(Di). 
Table 2. EMP analyses of clinopyroxene of selected Hole $738 \mathrm{C}$ samples. Calculations based on $\mathrm{O}=6$.

\begin{tabular}{|c|c|c|c|c|c|c|c|c|}
\hline $\begin{array}{l}\text { Core, Section, } \\
\text { Interval }[\mathrm{cm}]\end{array}$ & $\begin{array}{c}33 R-1 \\
2-4\end{array}$ & $\begin{array}{c}33 R-1 \\
2-4\end{array}$ & $\begin{array}{c}33 R-1 \\
4-4\end{array}$ & $\begin{array}{c}33 R-1 \\
2-4\end{array}$ & $\begin{array}{l}33 R-5 \\
30-32\end{array}$ & $\begin{array}{l}33 R-5 \\
30-32\end{array}$ & $\begin{array}{l}34 R-4 \\
39-42\end{array}$ & $\begin{array}{c}36 \mathrm{R}-1 \\
148- \\
150 \\
\end{array}$ \\
\hline $\mathrm{SiO}_{2}$ & 51.28 & 49.31 & 51.22 & 50.15 & 48.90 & 49.26 & 50.56 & 50.50 \\
\hline $\mathrm{TiO}_{2}$ & 0.40 & 0.93 & 0.47 & 0.74 & 1.02 & 0.99 & 0.85 & 0.76 \\
\hline $\mathrm{Al}_{2} \mathrm{O}_{3}$ & 2.00 & 3.21 & 2.12 & 3.07 & 1.57 & 1.30 & 2.18 & 1.95 \\
\hline $\mathrm{Fe}_{2} \mathrm{O}_{3}$ & 1.52 & 2.98 & 1.65 & 2.04 & 2.87 & 2.53 & 1.39 & 1.11 \\
\hline $\mathrm{FeO}$ & 5.58 & 5.51 & 5.26 & 7.86 & 13.75 & 14.03 & 13.67 & 11.39 \\
\hline $\mathrm{MnO}$ & 0.25 & 0.25 & 0.19 & 0.30 & 0.42 & 0.49 & 0.33 & 0.26 \\
\hline $\mathrm{MgO}$ & 17.33 & 15.52 & 17.33 & 17.44 & 12.71 & 12.88 & 16.84 & 14.88 \\
\hline $\mathrm{CaO}$ & 18.61 & 19.59 & 18.76 & 15.92 & 16.66 & 16.52 & 12.96 & 17.47 \\
\hline $\mathrm{Na}_{2} \mathrm{O}$ & 0.23 & 0.27 & 0.27 & 0.19 & 0.25 & 0.23 & 0.20 & 0.27 \\
\hline $\mathrm{K}_{2} \mathrm{O}$ & 0.00 & 0.01 & 0.01 & 0.01 & 0.00 & 0.01 & 0.04 & 0.02 \\
\hline $\mathrm{Cr}_{2} \mathrm{O}_{3}$ & 1.04 & 0.57 & 1.15 & 0.42 & 0.00 & 0.03 & 0.00 & 0.05 \\
\hline Total & 98.23 & 98.16 & 98.42 & 98.14 & 98.16 & 98.29 & 99.02 & 98.65 \\
\hline $\mathrm{Si}$ & 1.92 & 1.86 & 1.91 & 1.88 & 1.90 & 1.91 & 1.91 & 1.91 \\
\hline $\mathrm{Ti}$ & 0.01 & 0.03 & 0.01 & 0.02 & 0.03 & 0.03 & 0.02 & 0.02 \\
\hline $\mathrm{Al}$ & 0.09 & 0.14 & 0.09 & 0.14 & 0.07 & 0.06 & 0.10 & 0.09 \\
\hline $\mathrm{Fe}^{3+}$ & 0.04 & 0.08 & 0.05 & 0.06 & 0.08 & 0.07 & 0.04 & 0.03 \\
\hline $\mathrm{Fe}^{2+}$ & 0.17 & 0.17 & 0.16 & 0.25 & 0.45 & 0.46 & 0.43 & 0.36 \\
\hline $\mathrm{Mn}$ & 0.01 & 0.01 & 0.01 & 0.01 & 0.01 & 0.02 & 0.01 & 0.01 \\
\hline $\mathrm{Mg}$ & 0.97 & 0.87 & 0.96 & 0.98 & 0.74 & 0.75 & 0.95 & 0.84 \\
\hline $\mathrm{Ca}$ & 0.75 & 0.79 & 0.75 & 0.64 & 0.69 & 0.69 & 0.52 & 0.71 \\
\hline $\mathrm{Na}$ & 0.02 & 0.02 & 0.02 & 0.01 & 0.02 & 0.02 & 0.01 & 0.02 \\
\hline $\mathrm{K}$ & 0.00 & 0.00 & 0.00 & 0.00 & 0.00 & 0.00 & 0.00 & 0.00 \\
\hline $\mathrm{Cr}$ & 0.03 & 0.02 & 0.03 & 0.01 & 0.00 & 0.00 & 0.00 & 0.00 \\
\hline Enstatite & 48.26 & 43.66 & 48.16 & 48.81 & 36.82 & 37.28 & 47.30 & 41.95 \\
\hline Ferrosilite & 9.11 & 9.09 & 8.51 & 12.82 & 23.04 & 23.60 & 22.06 & 18.42 \\
\hline Wollastonite & 32.80 & 34.00 & 32.66 & 26.94 & 31.29 & 31.45 & 23.17 & 32.91 \\
\hline $\begin{array}{l}\text { Core, Section, } \\
\text { Interval }[\mathrm{cm}]\end{array}$ & $\begin{array}{c}36 \mathrm{R}-1 \\
148-150\end{array}$ & $\begin{array}{c}36 \mathrm{R}-1 \\
148-150\end{array}$ & $\begin{array}{c}36 \mathrm{R}-2 \\
143-146\end{array}$ & $\begin{array}{c}36 R-2 \\
143-146\end{array}$ & $\begin{array}{c}36 \mathrm{R}-2 \\
143-146\end{array}$ & $\begin{array}{c}36 R-2 \\
143-146\end{array}$ & $\begin{array}{c}36 R-2 \\
143-146\end{array}$ & $\begin{array}{c}36 \mathrm{R}-2 \\
143- \\
146\end{array}$ \\
\hline $\mathrm{SiO}_{2}$ & 49.96 & 49.82 & 51.54 & 53.31 & 51.42 & 51.81 & 52.03 & 50.71 \\
\hline $\mathrm{TiO}_{2}$ & 0.85 & 0.97 & 0.68 & 0.38 & 0.74 & 1.05 & 0.56 & 0.86 \\
\hline $\mathrm{Al}_{2} \mathrm{O}_{3}$ & 1.99 & 1.97 & 1.41 & 0.83 & 1.62 & 2.01 & 1.79 & 1.49 \\
\hline $\mathrm{Fe}_{2} \mathrm{O}_{3}$ & 1.11 & 1.49 & 1.53 & 1.97 & 3.25 & 2.35 & 2.38 & 2.23 \\
\hline $\mathrm{FeO}$ & 13.35 & 12.78 & 11.53 & 14.95 & 8.60 & 9.15 & 7.50 & 13.03 \\
\hline $\mathrm{MnO}$ & 0.35 & 0.32 & 0.30 & 0.43 & 0.13 & 0.31 & 0.17 & 0.23 \\
\hline $\mathrm{MgO}$ & 14.08 & 13.85 & 16.13 & 23.52 & 16.14 & 18.61 & 16.84 & 14.47 \\
\hline $\mathrm{CaO}$ & 16.57 & 17.05 & 16.24 & 4.91 & 18.40 & 14.90 & 18.81 & 16.74 \\
\hline $\mathrm{Na}_{2} \mathrm{O}$ & 0.23 & 0.30 & 0.14 & 0.06 & 0.20 & 0.19 & 0.18 & 0.15 \\
\hline $\mathrm{K}_{2} \mathrm{O}$ & 0.02 & 0.01 & 0.00 & 0.00 & 0.01 & 0.03 & 0.00 & 0.02 \\
\hline $\mathrm{Cr}_{2} \mathrm{O}_{3}$ & 0.02 & 0.00 & nd & nd & nd & nd & nd & nd \\
\hline Total & 98.52 & 98.56 & 99.50 & 100.36 & 100.51 & 100.42 & 100.26 & 99.93 \\
\hline $\mathrm{Si}$ & 1.91 & 1.90 & 1.93 & 1.95 & 1.91 & 1.90 & 1.92 & 1.92 \\
\hline $\mathrm{Ti}$ & 0.02 & 0.03 & 0.02 & 0.01 & 0.02 & 0.03 & 0.02 & 0.02 \\
\hline $\mathrm{Al}$ & 0.09 & 0.09 & 0.06 & 0.04 & 0.07 & 0.09 & 0.08 & 0.07 \\
\hline $\mathrm{Fe}^{3+}$ & 0.03 & 0.04 & 0.04 & 0.05 & 0.09 & 0.07 & 0.07 & 0.06 \\
\hline $\mathrm{Fe}^{2+}$ & 0.43 & 0.41 & 0.36 & 0.46 & 0.27 & 0.28 & 0.23 & 0.41 \\
\hline $\mathrm{Mn}$ & 0.01 & 0.01 & 0.01 & 0.01 & 0.00 & 0.01 & 0.01 & 0.01 \\
\hline $\mathrm{Mg}$ & 0.80 & 0.79 & 0.90 & 1.28 & 0.89 & 1.02 & 0.93 & 0.82 \\
\hline $\mathrm{Ca}$ & 0.68 & 0.70 & 0.65 & 0.19 & 0.73 & 0.59 & 0.74 & 0.68 \\
\hline $\mathrm{Na}$ & 0.02 & 0.02 & 0.01 & 0.00 & 0.01 & 0.01 & 0.01 & 0.01 \\
\hline K & 0.00 & 0.00 & 0.00 & 0.00 & 0.00 & 0.00 & 0.00 & 0.00 \\
\hline $\mathrm{Cr}$ & 0.00 & 0.00 & nd & nd & nd & nd & nd & nd \\
\hline Enstatite & 40.06 & 39.45 & 45.09 & 64.01 & 44.59 & 50.93 & 46.29 & 40.76 \\
\hline Ferrosilite & 21.89 & 20.93 & 18.56 & 23.49 & 13.53 & 14.53 & 11.84 & 20.96 \\
\hline Wollastonite & 31.31 & 32.17 & 30.25 & 7.47 & 32.88 & 25.88 & 33.89 & 30.95 \\
\hline
\end{tabular}


Table 3. EMP analyses of sheet silicates of selected Hole $738 \mathrm{C}$ samples. Calculations based on $\mathrm{O}=22$ and iron as Fe ${ }^{2+} .1-9=$ celadonite, $10-14=$ green saponite, $15-24$ = brown saponite, $25-38$ = ?montmorillonite, $39-44$ = various brown sheet silicates, $45-47$ = olivine-pseudomorphs.

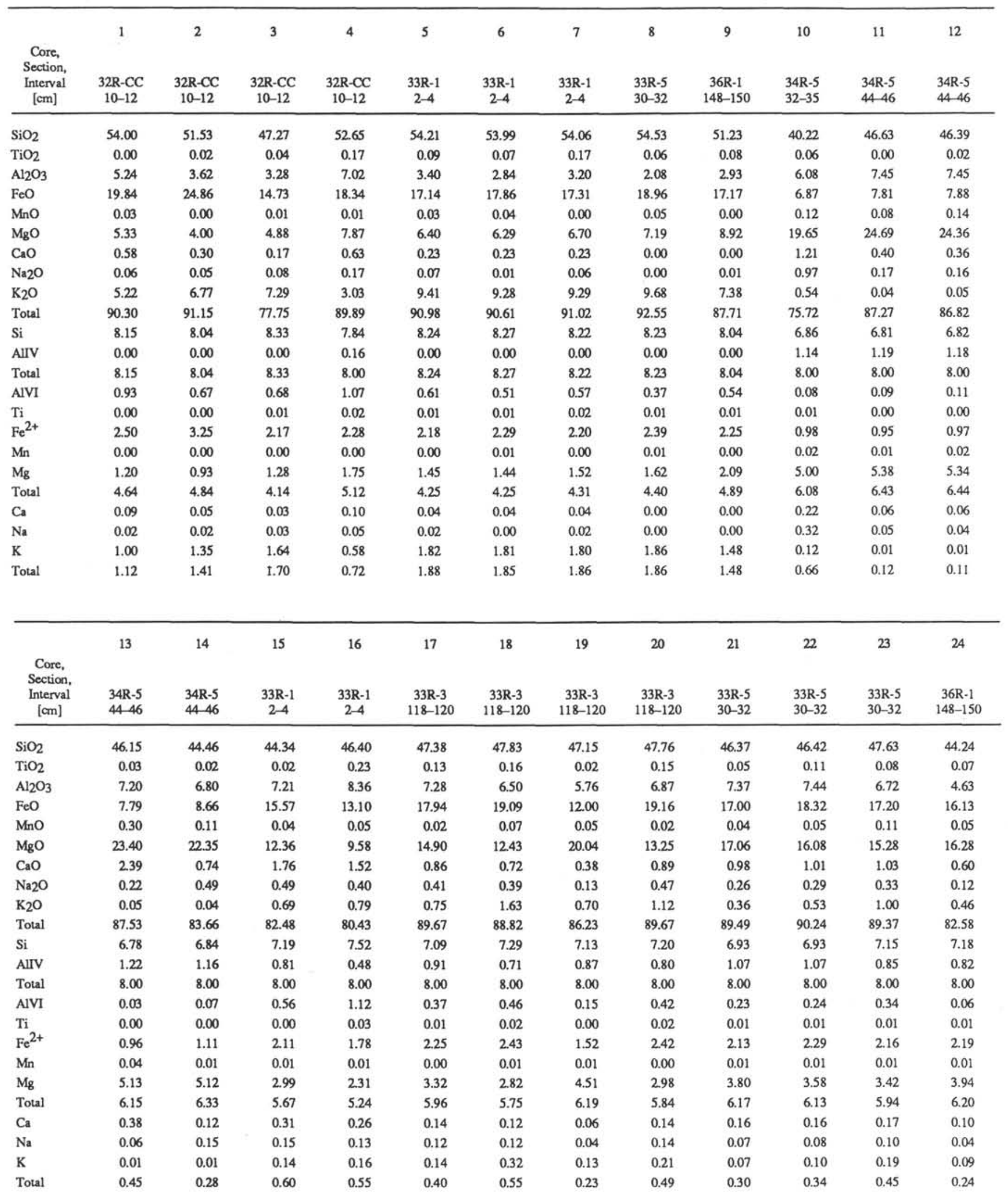


Table 3. (continued).

\begin{tabular}{|c|c|c|c|c|c|c|c|c|c|c|c|c|}
\hline $\begin{array}{l}\text { Core, } \\
\text { Section, } \\
\text { Interval } \\
\text { [cm] }\end{array}$ & $\begin{array}{c}32 \mathrm{R}-\mathrm{CC} \\
10-12\end{array}$ & $\begin{array}{c}32 \mathrm{R}-\mathrm{CC} \\
10-12\end{array}$ & $\begin{array}{c}32 \mathrm{R}-\mathrm{CC} \\
10-12\end{array}$ & $\begin{array}{c}32 \mathrm{R}-\mathrm{CC} \\
10-12\end{array}$ & $\begin{array}{c}32 R-C C \\
10-12\end{array}$ & $\begin{array}{l}34 R-5 \\
44-46\end{array}$ & $\begin{array}{l}34 R-5 \\
44-46\end{array}$ & $\begin{array}{l}34 R-5 \\
44-46\end{array}$ & $\begin{array}{l}34 R-5 \\
44-46\end{array}$ & $\begin{array}{l}34 R-5 \\
44-46\end{array}$ & $\begin{array}{l}34 R-5 \\
44-46\end{array}$ & $\begin{array}{l}34 \mathrm{R}-5 \\
44-46\end{array}$ \\
\hline $\mathrm{SiO}_{2}$ & 61.08 & 59.37 & 59.80 & 59.02 & 61.10 & 60.53 & 56.55 & 56.46 & 61.79 & 56.83 & 60.31 & 57.89 \\
\hline $\mathrm{TiO}_{2}$ & 0.26 & 0.77 & 0.93 & 0.79 & 0.47 & 0.46 & 0.90 & 0.78 & 0.36 & 1.55 & 0.60 & 0.38 \\
\hline $\mathrm{Al}_{2} \mathrm{O}_{3}$ & 17.47 & 19.20 & 18.46 & 17.79 & 18.19 & 16.49 & 14.68 & 13.86 & 17.64 & 13.43 & 17.12 & 14.51 \\
\hline $\mathrm{FeO}$ & 3.80 & 3.00 & 3.79 & 4.98 & 2.96 & 4.11 & 5.72 & 6.43 & 2.59 & 6.75 & 3.69 & 5.17 \\
\hline $\mathrm{MnO}$ & 0.07 & 0.00 & 0.10 & 0.14 & 0.11 & 0.06 & 0.10 & 0.07 & 0.04 & 0.16 & 0.05 & 0.04 \\
\hline $\mathrm{MgO}$ & 6.99 & 5.91 & 5.84 & 5.88 & 6.92 & 8.88 & 8.42 & 9.21 & 8.82 & 9.71 & 7.90 & 10.37 \\
\hline $\mathrm{CaO}$ & 0.89 & 0.98 & 0.89 & 0.83 & 0.96 & 0.71 & 2.36 & 1.50 & 0.77 & 1.58 & 0.58 & 1.09 \\
\hline $\mathrm{Na}_{2} \mathrm{O}$ & 0.18 & 0.16 & 0.28 & 0.26 & 0.15 & 0.38 & 0.94 & 0.87 & 0.29 & 1.14 & 0.56 & 0.65 \\
\hline $\mathrm{K}_{2} \mathrm{O}$ & 1.00 & 0.75 & 1.38 & 1.48 & 1.15 & 1.56 & 2.89 & 2.93 & 1.43 & 2.90 & 2.51 & 2.13 \\
\hline Total & 91.74 & 90.14 & 91.47 & 91.17 & 92.01 & 93.19 & 92.55 & 92.11 & 93.71 & 94.04 & 93.30 & 92.22 \\
\hline $\mathrm{Si}$ & 7.96 & 7.83 & 7.85 & 7.83 & 7.91 & 7.84 & 7.61 & 7.65 & 7.87 & 7.57 & 7.82 & 7.71 \\
\hline AIIV & 0.04 & 0.17 & 0.15 & 0.17 & 0.09 & 0.16 & 0.39 & 0.35 & 0.13 & 0.43 & 0.18 & 0.29 \\
\hline Total & 8.00 & 8.00 & 8.00 & 8.00 & 8.00 & 8.00 & 8.00 & 8.00 & 8.00 & 8.00 & 8.00 & 8.00 \\
\hline AlvI & 2.64 & 2.81 & 2.70 & 2.61 & 2.69 & 2.35 & 1.94 & 1.86 & 2.52 & 1.68 & 2.44 & 1.99 \\
\hline $\mathrm{Ti}$ & 0.03 & 0.08 & 0.09 & 0.08 & 0.05 & 0.04 & 0.09 & 0.08 & 0.03 & 0.15 & 0.06 & 0.04 \\
\hline $\mathrm{Fe}^{2+}$ & 0.41 & 0.33 & 0.42 & 0.55 & 0.32 & 0.44 & 0.64 & 0.73 & 0.28 & 0.75 & 0.40 & 0.58 \\
\hline Mn & 0.01 & 0.00 & 0.01 & 0.02 & 0.01 & 0.01 & 0.01 & 0.01 & 0.00 & 0.02 & 0.01 & 0.00 \\
\hline $\mathrm{Mg}$ & 1.36 & 1.16 & 1.14 & 1.16 & 1.34 & 1.71 & 1.69 & 1.86 & 1.67 & 1.93 & 1.53 & 2.06 \\
\hline Total & 4.45 & 4.38 & 4.36 & 4.42 & 4.40 & 4.56 & 4.37 & 4.54 & 4.51 & 4.53 & 4.43 & 4.66 \\
\hline $\mathrm{Ca}$ & 0.12 & 0.14 & 0.13 & 0.12 & 0.13 & 0.10 & 0.34 & 0.22 & 0.11 & 0.23 & 0.08 & 0.15 \\
\hline $\mathrm{Na}$ & 0.05 & 0.04 & 0.07 & 0.07 & 0.04 & 0.10 & 0.24 & 0.23 & 0.07 & 0.29 & 0.14 & 0.17 \\
\hline K & 0.17 & 0.13 & 0.23 & 0.25 & 0.19 & 0.26 & 0.50 & 0.51 & 0.23 & 0.49 & 0.41 & 0.36 \\
\hline Total & 0.34 & 0.31 & 0.43 & 0.44 & 0.36 & 0.45 & 1.08 & 0.95 & 0.41 & 1.01 & 0.64 & 0.69 \\
\hline
\end{tabular}

\begin{tabular}{|c|c|c|c|c|c|c|c|c|c|c|c|}
\hline $\begin{array}{l}\text { Core, } \\
\text { Section, } \\
\text { Interval } \\
{[\mathrm{cm}]}\end{array}$ & $\begin{array}{l}34 R-5 \\
44-46\end{array}$ & $\begin{array}{l}34 R-5 \\
44-46\end{array}$ & $\begin{array}{c}32 R-C C \\
10-12\end{array}$ & $\begin{array}{c}32 \mathrm{R}-\mathrm{CC} \\
10-12\end{array}$ & $\begin{array}{c}32 \mathrm{R}-\mathrm{CC} \\
10-12\end{array}$ & $\begin{array}{c}32 \mathrm{R}-\mathrm{CC} \\
10-12\end{array}$ & $\begin{array}{c}36 \mathrm{R}-1 \\
148-150\end{array}$ & $\begin{array}{c}36 \mathrm{R}-1 \\
148-150\end{array}$ & $\begin{array}{l}35 R-3 \\
15-18\end{array}$ & $\begin{array}{l}35 \mathrm{R}-3 \\
15-18\end{array}$ & $\begin{array}{l}35 \mathrm{R}-3 \\
15-18\end{array}$ \\
\hline $\mathrm{SiO}_{2}$ & 58.35 & 58.02 & 57.77 & 57.75 & 56.66 & 52.67 & 58.97 & 57.73 & 40.19 & 48.65 & 46.68 \\
\hline $\mathrm{TiO}_{2}$ & 0.48 & 0.35 & 0.43 & 0.07 & 0.11 & 0.08 & 0.45 & 0.26 & 0.76 & 0.43 & 0.29 \\
\hline $\mathrm{Al}_{2} \mathrm{O}_{3}$ & 15.35 & 14.80 & 14.52 & 13.30 & 9.75 & 8.30 & 13.24 & 11.92 & 9.86 & 7.83 & 8.49 \\
\hline $\mathrm{FeO}$ & 4.82 & 5.26 & 10.48 & 10.19 & 14.19 & 16.67 & 7.01 & 7.59 & 18.07 & 13.02 & 10.36 \\
\hline $\mathrm{MnO}$ & 0.08 & 0.16 & 0.02 & 0.05 & 0.02 & 0.00 & 0.08 & 0.00 & 0.02 & 0.06 & 0.01 \\
\hline $\mathrm{MgO}$ & 8.53 & 10.22 & 5.99 & 7.02 & 8.31 & 9.49 & 9.22 & 9.60 & 4.70 & 5.95 & 6.22 \\
\hline $\mathrm{CaO}$ & 0.91 & 1.22 & 1.03 & 0.99 & 0.91 & 0.74 & 0.92 & 0.86 & 0.71 & 1.03 & 0.85 \\
\hline $\mathrm{Na}_{2} \mathrm{O}$ & 0.53 & 0.61 & 0.21 & 0.14 & 0.11 & 0.37 & 0.28 & 0.27 & 1.03 & 1.01 & 0.83 \\
\hline $\mathrm{K}_{2} \mathrm{O}$ & 2.24 & 1.84 & 1.11 & 0.96 & 0.61 & 1.54 & 1.01 & 1.26 & 1.29 & 1.35 & 1.20 \\
\hline Total & 91.28 & 92.49 & 91.56 & 90.47 & 90.67 & 89.86 & 91.17 & 89.48 & 76.63 & 79.33 & 74.93 \\
\hline $\mathrm{Si}$ & 7.81 & 7.70 & 7.87 & 7.94 & 7.97 & 7.71 & 7.93 & 7.96 & 7.15 & 7.96 & 7.95 \\
\hline AllV & 0.19 & 0.30 & 0.13 & 0.06 & 0.03 & 0.29 & 0.07 & 0.04 & 0.85 & 0.04 & 0.05 \\
\hline Total & 8.00 & 8.00 & 8.00 & 8.00 & 8.00 & 8.00 & 8.00 & 8.00 & 8.00 & 8.00 & 8.00 \\
\hline AlVI & 2.24 & 2.02 & 2.20 & 2.10 & 1.58 & 1.14 & 2.02 & 1.89 & 1.22 & 1.46 & 1.65 \\
\hline $\mathrm{Ti}$ & 0.05 & 0.03 & 0.04 & 0.01 & 0.01 & 0.01 & 0.05 & 0.03 & 0.10 & 0.05 & 0.04 \\
\hline $\mathrm{Fe}^{2+}$ & 0.54 & 0.58 & 1.19 & 1.17 & 1.67 & 2.04 & 0.79 & 0.87 & 2.69 & 1.78 & 1.47 \\
\hline $\mathrm{Mn}$ & 0.01 & 0.02 & 0.00 & 0.01 & 0.00 & 0.00 & 0.01 & 0.00 & 0.00 & 0.01 & 0.00 \\
\hline $\mathrm{Mg}$ & 1.70 & 2.02 & 1.22 & 1.44 & 1.74 & 2.07 & 1.85 & 1.97 & 1.25 & 1.45 & 1.58 \\
\hline Total & 4.53 & 4.68 & 4.65 & 4.72 & 5.01 & 5.26 & 4.71 & 4.77 & 5.26 & 4.76 & 4.74 \\
\hline $\mathrm{Ca}$ & 0.13 & 0.17 & 0.15 & 0.15 & 0.14 & 0.12 & 0.13 & 0.13 & 0.14 & 0.18 & 0.16 \\
\hline $\mathrm{Na}$ & 0.14 & 0.16 & 0.06 & 0.04 & 0.03 & 0.10 & 0.07 & 0.07 & 0.36 & 0.32 & 0.27 \\
\hline K & 0.38 & 0.31 & 0.19 & 0.17 & 0.11 & 0.29 & 0.17 & 0.22 & 0.29 & 0.28 & 0.26 \\
\hline Total & 0.65 & 0.64 & 0.40 & 0.35 & 0.28 & 0.51 & 0.38 & 0.42 & 0.78 & 0.78 & 0.69 \\
\hline
\end{tabular}



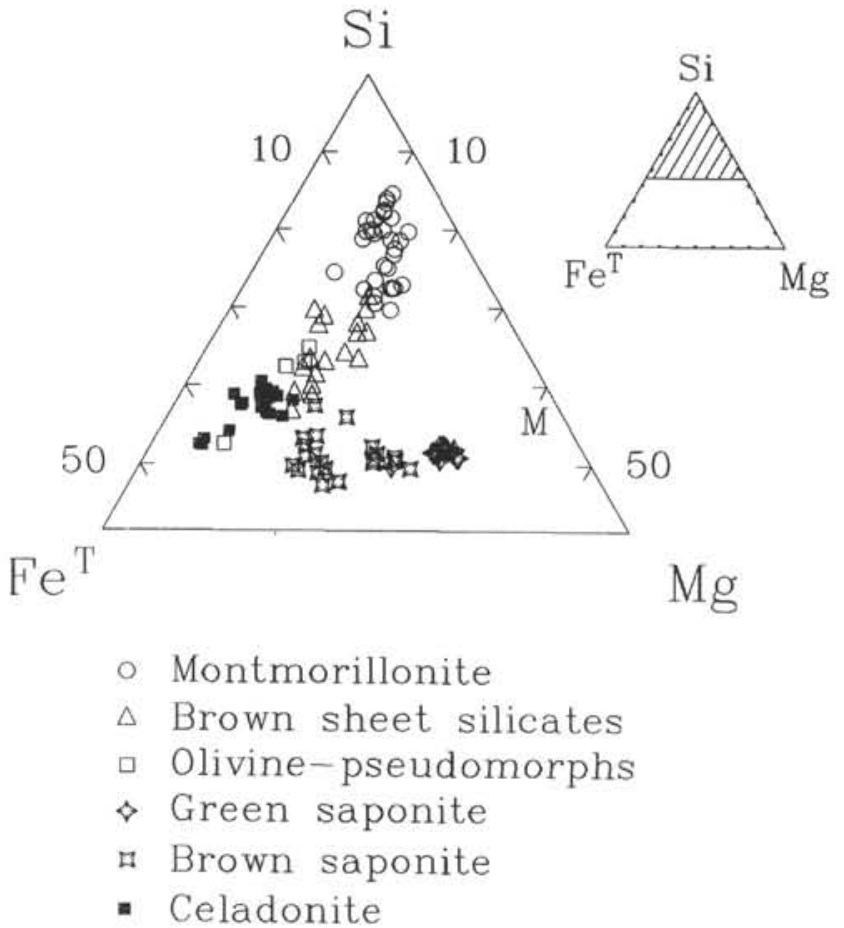

Figure 5. Compositional variation of sheet silicates from altered samples.
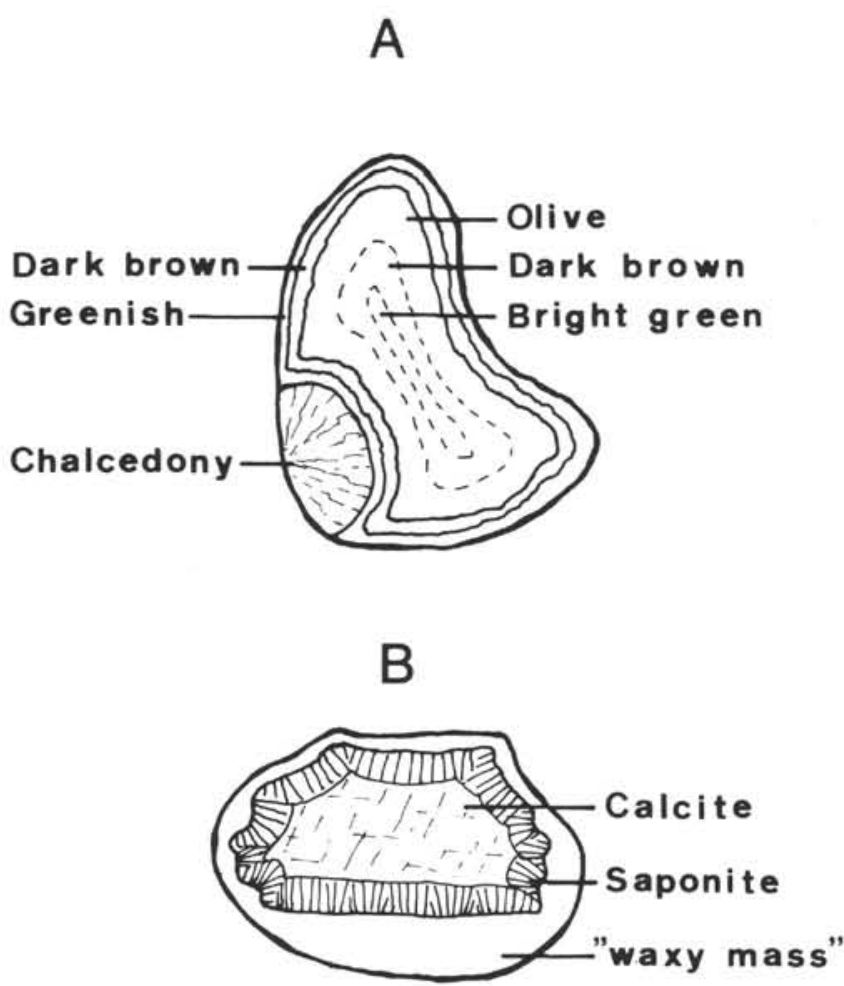

Figure 6. Sketch illustrating typical vesicle fillings. A. Vesicle filled by chalcedony and sheet minerals. Concentric layers of sheet minerals (greenish and dark brown) have sharp boundaries. The inner filling consists of varicolored sheet minerals grading from olive to dark brown to bright green, of which the latter is celadonite. B. Vesicle filled by "waxy mass" (showing geopetal texture in the lower part), radially arranged fibrous green saponite crystals, and blocky calcite.

\section{Element Variation with $\mathrm{H}_{2} \mathrm{O}$ as an Alteration Index}

Elements and element ratios plotted against $\mathrm{H}_{2} \mathrm{O}$ as an index for alteration (Fig. 10) show positive correlations for $\mathrm{Sr}, \mathrm{CO}_{2}$, and $\mathrm{Fe}_{2} \mathrm{O}_{3} / \mathrm{FeO}$, and negative correlations for $\mathrm{CaO}$. $\mathrm{Na}_{2} \mathrm{O}$ and $\mathrm{K}_{2} \mathrm{O}$ also seem to increase with increasing $\mathrm{H}_{2} \mathrm{O}$. In comparison with the massive flow basalt samples, the clastic samples have distinctly higher $\mathrm{FeO}^{\mathrm{T}}$, $\mathrm{CO}_{2}, \mathrm{H}_{2} \mathrm{O}$, and $\mathrm{K}_{2} \mathrm{O}$ concentrations, and higher $\mathrm{K} / \mathrm{Rb}, \mathrm{K} / \mathrm{Sr}$, and $\mathrm{K} / \mathrm{Ba}$ ratios. In contrast, $\mathrm{CaO}, \mathrm{Sr}$, and $\mathrm{V}$ concentrations are lower (Fig. 10). The variations in these elements, and to a lower degree in $\mathrm{Si}, \mathrm{Ba}, \mathrm{Y}$, $\mathrm{Rb}$, and $\mathrm{MgO}$ do not systematically vary with depth, but with the degree of alteration, a measure of which is the increase in $\mathrm{Fe}_{2} \mathrm{O}_{3}, \mathrm{~K} / \mathrm{Rb}$, $\mathrm{CO}_{2}$, or $\mathrm{H}_{2} \mathrm{O}$.

\section{Rare Earth Element Concentrations}

The REE of the least altered basalts (Fig. 11, Table 7) display a significant enrichment of the LREE over the HREE $\left(\mathrm{Ce}_{\mathrm{cn}} / \mathrm{Yb} \mathrm{b}_{\mathrm{cn}}=\right.$ 3.2-4.1), and have smooth and very similar chondrite normalized patterns without pronounced Eu-anomalies or flat HREE tails. Sample $119-738 \mathrm{C}-33 \mathrm{R}-1,2-4 \mathrm{~cm}$, from the top has a very slight positive Eu anomaly and the lowest REE concentrations. Sample 119-738C-33R$5,22-25 \mathrm{~cm}$, has the highest concentration in LREE and second lowest concentrations in HREE, while Sample 119-738C-36R-3, 37-40 cm, shows the second lowest LREE and higher HREE concentrations (Fig. 11).

\section{DISCUSSION}

\section{Lithostratigraphy}

The absence of glassy rinds, the large amount and size of vesicles (up to $25 \%$ ), the high degree of oxidation, the lack of intercalated marine sediments (carbonate, fossils), and the formation as aa-flows indicate subaerial to shallow water eruption and emplacement of the basalt flows and the breccias from Unit VIII. The roundness of the clasts and the sedimentary matrix of Subunit VIIa are evidence for reworking in a shallow marine environment.

The basalt layers represent aa-lava flows. Thin lava layers within the breccias belong to lava clinkers associated with aa-flows. Breccias dominated by vesicular tachylitic clasts may represent top breccias whereas the basaltic clast dominated breccias may represent basal breccias. Thickness of the breccia units and angularity of the lithoclasts are interpreted to indicate provenance from a proximal fracture zone where small volume lava flows and larger volumes of breccia were generated.

\section{Alteration}

In general, cold seawater alteration (e.g., Robinson et al., 1977; Donnelly et al., 1980; Lawrence, 1980; Staudigel and Hart, 1983; Bednarz and Schmincke, 1989) is characterized by a gain in $\mathrm{H}_{2} \mathrm{O}$, $\mathrm{K}_{2} \mathrm{O}, \mathrm{Ba}$, and $\mathrm{Rb}$, a high oxidation, and a loss of $\mathrm{CaO}$ and minor $\mathrm{Na}_{2} \mathrm{O}$, under temperatures lower than $40^{\circ} \mathrm{C}$ and not significantly different from the ocean bottom. Replacement of plagioclase by K-feldspar and formation of various sheet silicates and carbonate are the mineralogical expressions of the chemical changes.

Low-temperature alteration (e.g., Bednarz and Schmincke, 1989) is characterized by a small gain of $\mathrm{K}_{2} \mathrm{O}$, a gain of $\mathrm{Na}_{2} \mathrm{O}$, and $\mathrm{MgO}$, and less depletion of $\mathrm{CaO}$ than during cold seawater alteration. Celadonite and saponite replace interstitial glass and fill voids, but both are also reported as a result of cold seawater alteration (Andrews, 1980). Plagioclase and clinopyroxene are fresh or altered to smectite and smectite/chlorite mixed minerals. Calcite is less abundant than in the cold seawater alteration zone. The secondary mineral assemblage indicates temperatures below $200^{\circ} \mathrm{C}$.

Saponite and celadonite phases are typical mineral assemblages formed during cold seawater and low-temperature alteration at temperatures as low as $30^{\circ} \mathrm{C}$ (Seyfried et al., 1978; Andrews, 1979). Celadonite develops at oxidative conditions and a low $\mathrm{pH}$ from 
A

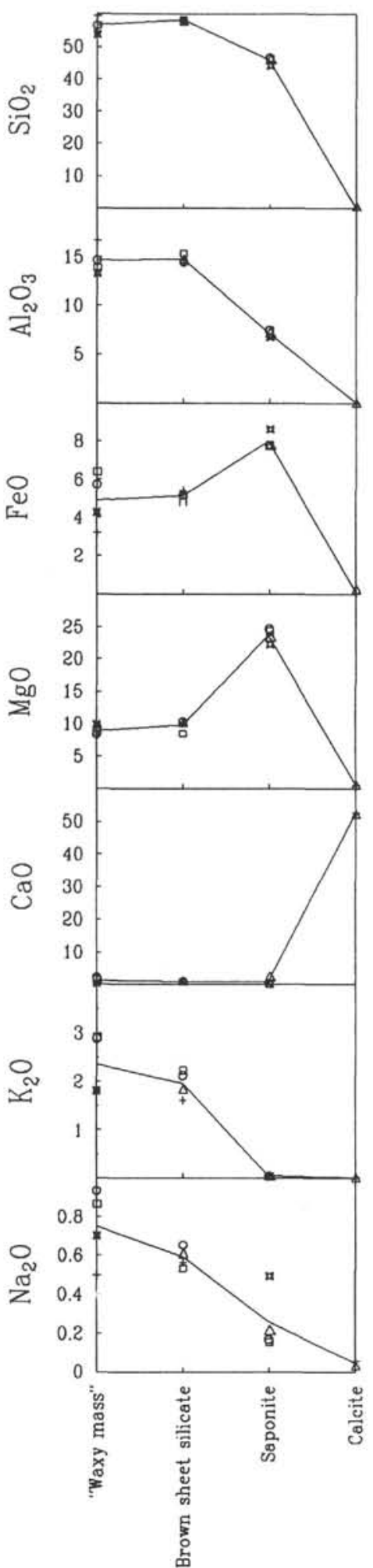

B

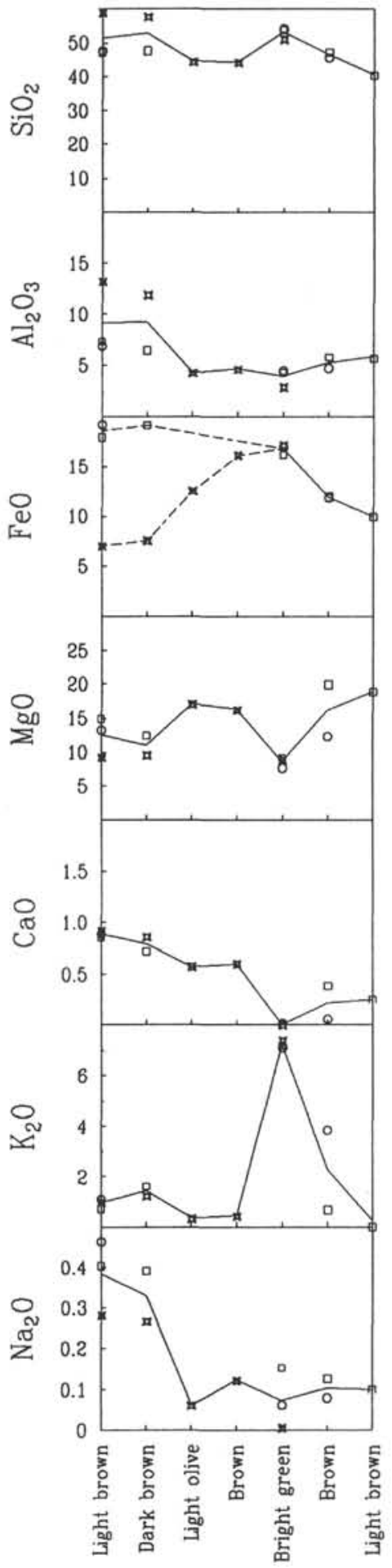

Figure 7. Variation of chemical composition of two distinct successions of secondary minerals in vesicles (left to right $=$ rim to core; lack of symbol = mineral absent). Oxides in wt\%. A. "Waxy mass"-light brown sheet silicate-green saponite-calcite. Four vesicles in Sample 119-738C-32R-5, $44-46 \mathrm{~cm}$. B. Concentric layers of varicolored sheet minerals in Samples 119-738C-33R-3, 118-120 cm (circle and square), and 119-738C-36R-1, 148-150 cm (star). 
Table 4. Major and trace element concentrations of basement samples and their ratios analyzed by XRF. VIIa $=$ Unit VIIa, VIIb $=$ Unit VIIb, $b=b a s a l t$ in Unit VIII, br = breccia in Unit VIII.

\begin{tabular}{|c|c|c|c|c|c|c|c|c|c|c|c|c|c|}
\hline \multicolumn{14}{|c|}{ Original values } \\
\hline $\begin{array}{l}\text { Core, } \\
\text { Section, } \\
\text { Interval } \\
\text { [cm] }\end{array}$ & \multicolumn{2}{|c|}{$\begin{array}{c}32 \mathrm{R}-\mathrm{CC} \\
0-4\end{array}$} & $\begin{array}{c}32 \mathrm{R}-\mathrm{CC} \\
12-14\end{array}$ & $\begin{array}{c}33 R-1 \\
2-4\end{array}$ & $\begin{array}{c}33 R-1 \\
129-132\end{array}$ & $\begin{array}{c}33 R-1 \\
134-137\end{array}$ & $\begin{array}{l}33 R-2 \\
59-62\end{array}$ & $\begin{array}{l}33 R-3 \\
22-25\end{array}$ & $\begin{array}{l}33 R-3 \\
38-41\end{array}$ & $\begin{array}{c}33 R-3 \\
130-133\end{array}$ & $\begin{array}{l}33 R-4 \\
68-71\end{array}$ & $\begin{array}{c}33 R-4 \\
111-114\end{array}$ & $\begin{array}{l}33 R-5 \\
22-25\end{array}$ \\
\hline $\begin{array}{l}\text { Rock } \\
\text { type }\end{array}$ & VII & & VIIb & b & br & $\mathrm{br}$ & br & br & $b r$ & br & br & br & b \\
\hline $\mathrm{SiO}_{2}$ & 44.5 & & 44.40 & 50.70 & 47.60 & 49.96 & 49.10 & 49.30 & 49.30 & 50.90 & 49.70 & 47.43 & 51.04 \\
\hline $\mathrm{TiO}_{2}$ & 2.1 & & 1.32 & 1.68 & 1.73 & 2.02 & 1.71 & 1.54 & 1.67 & 1.56 & 1.46 & 1.42 & 1.59 \\
\hline $\mathrm{Al}_{2} \mathrm{O}_{3}$ & 17.4 & & 15.83 & 16.05 & 14.35 & 16.28 & 13.77 & 14.51 & 15.59 & 14.92 & 13.85 & 13.41 & 15.41 \\
\hline $\mathrm{Fe}_{2} \mathrm{O}_{3}$ & 15.2 & & 8.98 & 6.13 & 11.00 & 9.04 & 9.42 & 8.88 & 8.00 & 7.54 & 8.69 & 8.42 & 5.27 \\
\hline $\mathrm{FeO}$ & 1.1 & & 1.36 & 4.17 & 1.87 & 2.89 & 2.63 & 2.40 & 3.04 & 3.27 & 2.94 & 3.02 & 4.64 \\
\hline $\mathrm{MnO}$ & 0.1 & & 0.08 & 0.16 & 0.11 & 0.09 & 0.14 & 0.11 & 0.14 & 0.14 & 0.16 & 0.17 & 0.20 \\
\hline $\mathrm{MgO}$ & 1.4 & & 2.06 & 5.49 & 4.39 & 4.00 & 5.27 & 5.54 & 5.14 & 5.40 & 5.34 & 5.33 & 5.62 \\
\hline $\mathrm{CaO}$ & 7.6 & & 11.69 & 9.35 & 7.79 & 6.83 & 7.43 & 7.71 & 8.78 & 6.78 & 7.59 & 10.21 & 9.10 \\
\hline $\mathrm{Na}_{2} \mathrm{O}$ & 3.1 & & 1.83 & 2.82 & 2.62 & 3.30 & 2.70 & 2.64 & 2.69 & 2.72 & 2.59 & 2.75 & 3.02 \\
\hline $\mathrm{K}_{2} \mathrm{O}$ & 1.2 & & 1.56 & 0.97 & 1.68 & 1.78 & 1.48 & 1.21 & 1.39 & 2.41 & 2.44 & 2.08 & 1.81 \\
\hline $\mathrm{P}_{2} \mathrm{O}_{5}$ & 0.4 & & 0.14 & 0.19 & 0.28 & 0.23 & 0.25 & 0.16 & 0.18 & 0.19 & 0.18 & 0.18 & 0.21 \\
\hline $\mathrm{Cr}$ & 142 & & 85 & 219 & 163 & 229 & 48 & 119 & 130 & 97 & 87 & 88 & 100 \\
\hline Co & 33 & & 31 & 46 & 51 & 42 & 39 & 40 & 47 & 45 & 47 & 40 & 42 \\
\hline $\mathrm{Ba}$ & 322 & & 217 & 197 & 301 & 271 & 203 & 169 & 204 & 310 & 269 & 264 & 303 \\
\hline v & 307 & & 104 & 292 & 211 & 307 & 237 & 236 & 292 & 268 & 243 & 255 & 286 \\
\hline $\mathrm{Ni}$ & 78 & & 41 & 36 & 59 & 35 & 35 & 34 & 38 & 33 & 38 & 35 & 42 \\
\hline $\mathrm{Cu}$ & 26 & & 31 & 35 & 71 & 69 & 43 & 28 & 31 & 27 & 40 & 57 & 23 \\
\hline $\mathrm{Zn}$ & 259 & & 99 & 118 & 98 & 116 & 108 & 99 & 130 & 99 & 95 & 101 & 99 \\
\hline $\mathrm{Rb}$ & 21 & & 23 & 15 & 13 & 26 & 20 & 14 & 15 & 40 & 45 & 35 & 30 \\
\hline St & 362 & & 293 & 310 & 287 & 322 & 304 & 303 & 307 & 271 & 257 & 253 & 269 \\
\hline$Y$ & 55 & & 23 & 29 & 38 & 22 & 47 & 35 & 33 & 29 & 30 & 34 & 33 \\
\hline $\mathrm{Zr}$ & 187 & & 137 & 154 & 161 & 164 & 157 & 136 & 142 & 140 & 129 & 127 & 140 \\
\hline $\mathrm{Nb}$ & 12 & & 8 & 10 & 9 & 9 & 8 & 7 & 8 & 8 & 8 & 7 & 8 \\
\hline $\mathrm{CO}_{2}$ & 1.8 & & 4.97 & 0.14 & 2.17 & 1.27 & 3.29 & 2.21 & 2.11 & 0.21 & 2.28 & 3.63 & 0.22 \\
\hline $\mathrm{H}_{2} \mathrm{O}$ & 4.3 & & 4.52 & 2.34 & 3.07 & 3.47 & 3.29 & 3.42 & 2.57 & 2.77 & 3.14 & 2.70 & 1.43 \\
\hline Sum & 100.9 & & 98.85 & 100.34 & 98.80 & 101.32 & 100.61 & 99.75 & 100.74 & 98.94 & 100.49 & 100.88 & 99.70 \\
\hline $\begin{array}{l}\mathrm{Fe}_{2} \mathrm{O}_{3} / \mathrm{Fe} \\
\mathrm{O}\end{array}$ & 13.1 & & 6.62 & 1.47 & 5.89 & 3.13 & 3.59 & 3.69 & 2.63 & 2.31 & 2.95 & 2.78 & 1.14 \\
\hline $\mathrm{Zr} / \mathrm{Nb}$ & 15.5 & & 17.13 & 15.40 & 17.89 & 18.22 & 19.63 & 19.43 & 17.75 & 17.50 & 16.13 & 18.14 & 17.50 \\
\hline $\mathrm{Ti} / \mathrm{Zr}$ & 68.9 & & 57.76 & 65.40 & 64.42 & 73.84 & 65.30 & 67.88 & 70.50 & 66.80 & 67.85 & 67.03 & 68.09 \\
\hline $\mathrm{Z} r \mathrm{Y}$ & 3.4 & & 5.96 & 5.31 & 4.24 & 7.45 & 3.34 & 3.89 & 4.30 & 4.83 & 4.30 & 3.74 & 4.24 \\
\hline $\mathrm{YNb}$ & 4.5 & & 2.88 & 2.90 & 4.22 & 2.44 & 5.88 & 5.00 & 4.13 & 3.63 & 3.75 & 4.86 & 4.13 \\
\hline $\mathrm{Ba} / \mathrm{Nb}$ & 26.8 & & 27.13 & 19.70 & 33.44 & 30.11 & 25.38 & 24.14 & 25.50 & 38.75 & 33.63 & 37.71 & 37,88 \\
\hline $\mathrm{K} / \mathrm{Ba}$ & 32.7 & & 59.68 & 40.88 & 46.33 & 54.53 & 60.52 & 59.44 & 56.56 & 64.54 & 75.30 & 65.41 & 49.59 \\
\hline $\mathrm{K} / \mathrm{Sr}$ & 29.1 & & 44.20 & 25.98 & 48.59 & 45.89 & 40.42 & 33.15 & 37.59 & 73.83 & 78.82 & 68.25 & 55.86 \\
\hline $\mathrm{K} / \mathrm{Rb}$ & 502.0 & & 563.07 & 536.84 & 1072.82 & 568.34 & 614.32 & 717.50 & 769.28 & 500.17 & 450.13 & 493.35 & 500.86 \\
\hline $\mathrm{Ti} / \mathrm{N}$ & 41.9 & & 76.09 & 34.49 & 49.15 & 39.45 & 43.26 & 39.12 & 34.29 & 34.90 & 36.02 & 33.38 & 33.33 \\
\hline $\mathrm{CO}_{2}$ - and 1 & $12 \mathrm{O}$-fre & e normaliz & zed values & & & & & & & & & & \\
\hline $\begin{array}{l}\text { Core, Sec } \\
\text { Interval [ }\end{array}$ & ion, & $\begin{array}{c}32 \mathrm{R}-\mathrm{CC} \\
0-4\end{array}$ & $\begin{array}{c}32 \mathrm{R}-\mathrm{CC} \\
12-14\end{array}$ & $\begin{array}{c}33 R-1 \\
2-4\end{array}$ & $\begin{array}{c}33 R-1 \\
129-132\end{array}$ & $\begin{array}{c}33 R-1 \\
134-137\end{array}$ & $\begin{array}{l}33 R-2 \\
59-62\end{array}$ & $\begin{array}{l}33 R-3 \\
22-25\end{array}$ & $\begin{array}{l}33 R-3 \\
38-41\end{array}$ & $\begin{array}{c}33 R-3 \\
130-133\end{array}$ & $\begin{array}{l}33 R-4 \\
68-71\end{array}$ & $\begin{array}{c}33 R-4 \\
111-114\end{array}$ & $\begin{array}{l}33 \mathrm{R}-5 \\
22-25\end{array}$ \\
\hline Rock ty & & $\mathrm{VHa}$ & VIIb & b & br & br & br & be & br & br & br & br & b \\
\hline $\mathrm{SiO}_{2}$ & & 46.96 & 49.69 & 51.81 & 50.88 & 51.73 & 52.22 & 52.38 & 51.32 & 53.04 & 52.28 & 50.16 & 52.05 \\
\hline $\mathrm{TiO}_{2}$ & & 2.27 & 1.48 & 1.72 & 1.85 & 2.09 & 1.82 & 1.64 & 1.74 & 1.63 & 1.54 & 1.50 & 1.62 \\
\hline $\mathrm{Al}_{2} \mathrm{O}_{3}$ & & 18.37 & 17.72 & 16.40 & 15.34 & 16.86 & 14.65 & 15.42 & 16.23 & 15.55 & 14.57 & 14.18 & 15.72 \\
\hline $\mathrm{Fe}_{2} \mathrm{O}_{3}$ & & 16.09 & 10.05 & 6.27 & 11.75 & 9.36 & 10.02 & 9.43 & 8.33 & 7.86 & 9.14 & 8.90 & 5.38 \\
\hline $\mathrm{FeO}$ & & 1.23 & 1.52 & 4.26 & 1.99 & 2.99 & 2.79 & 2.55 & 3.17 & 3.41 & 3.10 & 3.20 & 4.73 \\
\hline $\mathrm{MnO}$ & & 0.15 & 0.09 & 0.16 & 0.12 & 0.09 & 0.15 & 0.12 & 0.15 & 0.15 & 0.17 & 0.18 & 0.20 \\
\hline $\mathrm{MgO}$ & & 1.52 & 2.31 & 5.61 & 4.69 & 4.14 & 5.61 & 5.89 & 5.35 & 5.63 & 5.62 & 5.64 & 5.73 \\
\hline $\mathrm{CaO}$ & & 8.09 & 13.08 & 9.55 & 8.33 & 7.07 & 7.90 & 8.19 & 9.14 & 7.07 & 7.98 & 10.80 & 9.28 \\
\hline $\mathrm{Na}_{2} \mathrm{O}$ & & 3.27 & 2.05 & 2.88 & 2.80 & 3.42 & 2.87 & 2.80 & 2.80 & 2.83 & 2.72 & 2.91 & 3.08 \\
\hline $\mathrm{K}_{2} \mathrm{O}$ & & 1.34 & 1.75 & 0.99 & 1.80 & 1.84 & 1.57 & 1.29 & 1.45 & 2.51 & 2.57 & 2.20 & 1.85 \\
\hline $\mathrm{P}_{2} \mathrm{O}_{5}$ & & 0.52 & 0.16 & 0.19 & 0.30 & 0.24 & 0.27 & 0.17 & 0.19 & 0.20 & 0.19 & 0.19 & 0.21 \\
\hline $\mathrm{Cr}$ & & 150 & 95 & 224 & 174 & 237 & 51 & 126 & 135 & 101 & 92 & 93 & 102 \\
\hline Co & & 35 & 35 & 47 & 55 & 43 & 41 & 42 & 49 & 47 & 49 & 42 & 43 \\
\hline $\mathrm{Ba}$ & & 340 & 243 & 201 & 322 & 281 & 216 & 180 & 212 & 323 & 283 & 279 & 309 \\
\hline $\mathrm{v}$ & & 324 & 116 & 298 & 226 & 318 & 252 & 251 & 304 & 279 & 256 & 270 & 292 \\
\hline $\mathrm{Ni}$ & & 82 & 46 & 37 & 63 & 36 & 37 & 36 & 40 & 34 & 40 & $\cdot 37$ & 43 \\
\hline $\mathrm{Cu}$ & & 27 & 35 & 36 & 76 & 71 & 46 & 30 & 32 & 28 & 42 & 60 & 23 \\
\hline $\mathrm{Zn}$ & & 273 & 111 & 121 & 105 & 120 & 115 & 105 & 135 & 103 & 100 & 107 & 101 \\
\hline $\mathbf{R b}$ & & 22 & 26 & 15 & 14 & 27 & 21 & 15 & 16 & 42 & 47 & 37 & 31 \\
\hline Sr & & 382 & 328 & 317 & 307 & 333 & 323 & 322 & 320 & 282 & 270 & 268 & 274 \\
\hline $\mathrm{Y}$ & & 58 & 26 & 30 & 41 & 23 & 50 & 37 & 34 & 30 & 32 & 36 & 34 \\
\hline $\mathrm{Z} x$ & & 197 & 153 & 157 & 172 & 170 & 167 & 144 & 148 & 146 & 136 & 134 & 143 \\
\hline $\mathrm{Nb}$ & & 13 & 9 & 10 & 10 & 9 & 9 & 7 & 8 & 8 & 8 & 7 & 8 \\
\hline Normalizat & & 1.06 & 1.12 & 1.02 & 1.07 & 1.04 & 1.06 & 1.06 & 1.04 & 1.04 & 1.05 & 1.06 & 1.02 \\
\hline
\end{tabular}


Table 4. (continued).

\begin{tabular}{|c|c|c|c|c|c|c|c|c|c|c|c|c|}
\hline \multicolumn{13}{|c|}{ Original values } \\
\hline $\begin{array}{c}\text { Core, } \\
\text { Section, } \\
\text { Interval }[\mathrm{cm}]\end{array}$ & $\begin{array}{c}33 R-5 \\
109-112\end{array}$ & $\begin{array}{l}34 \mathrm{R}-1 \\
41-44\end{array}$ & $\begin{array}{c}34 \mathrm{R}-2 \\
108-111\end{array}$ & $\begin{array}{l}34 \mathrm{R}-3 \\
66-69\end{array}$ & $\begin{array}{l}34 R-4 \\
39-42\end{array}$ & $\begin{array}{l}34 R-4 \\
65-68\end{array}$ & $\begin{array}{l}34 R-5 \\
32-35\end{array}$ & $\begin{array}{l}34 R-6 \\
22-25\end{array}$ & $\begin{array}{l}34 R-6 \\
61-64\end{array}$ & $\begin{array}{l}34 \mathrm{R}-6 \\
93-96\end{array}$ & $\begin{array}{l}35 R-1 \\
88-91\end{array}$ & $\begin{array}{c}35 R-2 \\
144-147\end{array}$ \\
\hline Rock type & $b$ & br & b & b & b & br & $\mathrm{br}$ & b & $\mathrm{br}$ & b & b & b \\
\hline $\mathrm{SiO}_{2}$ & 49.80 & 51.20 & 52.00 & 42.90 & 50.60 & 45.90 & 46.70 & 50.00 & 44.90 & 46.20 & 50.20 & 49.80 \\
\hline $\mathrm{TiO}_{2}$ & 1.69 & 1.51 & 1.56 & 1.29 & 1.62 & 1.44 & 1.52 & 1.71 & 1.43 & 1.63 & 1.63 & 1.68 \\
\hline $\mathrm{Al}_{2} \mathrm{O}_{3}$ & 15.39 & 13.87 & 14.53 & 12.16 & 14.68 & 13.23 & 14.04 & 15.63 & 13.17 & 14.35 & 15.17 & 15.34 \\
\hline $\mathrm{Fe}_{2} \mathrm{O}_{3}$ & 8.01 & 8.27 & 3.98 & 7.30 & 6.77 & 10.76 & 11.31 & 5.48 & 11.09 & 6.57 & 6.32 & 7.90 \\
\hline $\mathrm{FeO}$ & 2.60 & 3.52 & 6.71 & 3.56 & 4.16 & 0.95 & 0.84 & 3.86 & 0.58 & 2.93 & 4.15 & 2.86 \\
\hline $\mathrm{MnO}$ & 0.12 & 0.11 & 0.20 & 0.13 & 0.17 & 0.16 & 0.18 & 0.21 & 0.18 & 0.20 & 0.13 & 0.11 \\
\hline $\mathrm{MgO}$ & 6.62 & 5.67 & 6.09 & 4.97 & 6.20 & 5.26 & 5.64 & 7.09 & 4.73 & 6.30 & 6.44 & 6.59 \\
\hline $\mathrm{CaO}$ & 7.82 & 6.43 & 9.47 & 14.48 & 8.79 & 11.71 & 9.50 & 8.64 & 10.47 & 9.86 & 9.07 & 7.92 \\
\hline $\mathrm{Na}_{2} \mathrm{O}$ & 2.82 & 2.63 & 2.58 & 2.66 & 2.64 & 2.53 & 2.49 & 2.83 & 1.93 & 2.60 & 2.64 & 2.81 \\
\hline $\mathrm{K}_{2} \mathrm{O}$ & 0.69 & 2.42 & 1.08 & 1.39 & 1.38 & 1.75 & 1.21 & 0.71 & 3.38 & 1.03 & 0.52 & 0.65 \\
\hline $\mathrm{P}_{2} \mathrm{O} 5$ & 0.17 & 0.22 & 0.19 & 0.18 & 0.19 & 0.16 & 0.13 & 0.21 & 0.17 & 0.19 & 0.16 & 0.14 \\
\hline $\mathrm{Cr}_{\mathrm{r}}$ & 99 & 80 & 104 & 75 & 97 & 89 & 73 & 92 & 72 & 84 & 94 & 93 \\
\hline Co & 44 & 36 & 41 & 36 & 43 & 39 & 41 & 57 & 33 & 45 & 51 & 44 \\
\hline $\mathrm{Ba}$ & 209 & 270 & 317 & 267 & 316 & 270 & 132 & 290 & 406 & 176 & 194 & 215 \\
\hline $\mathrm{v}$ & 303 & 243 & 271 & 247 & 277 & 190 & 162 & 309 & 149 & 274 & 290 & 309 \\
\hline $\mathrm{Ni}$ & 29 & 28 & 27 & 32 & 31 & 27 & 39 & 30 & 26 & 35 & 31 & 27 \\
\hline $\mathrm{Cu}$ & 29 & 66 & 32 & 76 & 74 & 163 & 63 & 85 & 38 & 144 & 100 & 31 \\
\hline $\mathrm{Zn}$ & 92 & 97 & 101 & 99 & 98 & 84 & 122 & 117 & 83 & 104 & 103 & 96 \\
\hline $\mathrm{Rb}$ & 11 & 41 & 27 & 20 & 23 & 9 & 5 & 9 & 11 & 11 & 5 & 11 \\
\hline $\mathrm{Sr}$ & 323 & 248 & 264 & 251 & 286 & 250 & 282 & 315 & 222 & 300 & 308 & 321 \\
\hline $\mathbf{Y}$ & 34 & 31 & 36 & 36 & 32 & 44 & 33 & 30 & 37 & 32 & 33 & 31 \\
\hline $\mathrm{Z}_{x}$ & 148 & 131 & 141 & 120 & 145 & 120 & 131 & 152 & 127 & 134 & 143 & 148 \\
\hline $\mathrm{Nb}$ & 9 & 6 & 9 & 10 & 9 & 1 & 6 & 8 & 7 & 9 & 8 & 9 \\
\hline $\mathrm{CO}_{2}$ & 0.94 & 1.53 & 0.07 & 6.62 & 0.47 & 4.22 & 3.50 & 1.06 & 4.75 & 3.67 & 0.21 & 0.67 \\
\hline $\mathrm{H}_{2} \mathrm{O}$ & 2.89 & 2.82 & 0.75 & 2.27 & 1.97 & 2.12 & 3.26 & 2.65 & 2.05 & 2.91 & 2.28 & 2.95 \\
\hline Sum & 99.69 & 100.33 & 99.34 & 100.04 & 99.78 & 100.32 & 100.44 & 100.23 & 98.95 & 98.57 & 99.05 & 99.55 \\
\hline $\mathrm{Fe}_{2} \mathrm{O}_{3} / \mathrm{FeO}$ & 3.08 & 2.35 & 0.59 & 2.05 & 1.63 & 11.35 & 13.45 & 1.42 & 19.27 & 2.24 & 1.52 & 2.76 \\
\hline $\mathrm{Z} r \mathrm{Nb}$ & 16.44 & 21.83 & 15.67 & 12.00 & 16.11 & 120.00 & 21.83 & 19.00 & 18.14 & 14.89 & 17.88 & 16.44 \\
\hline $\mathrm{Ti} / \mathrm{Rt}$ & 68.46 & 69.10 & 66.33 & 64.45 & 66.98 & 71.94 & 69.56 & 67.44 & 67.50 & 72.92 & 68.33 & 68.05 \\
\hline $\mathrm{Zr} / \mathrm{Y}$ & 4.35 & 4.23 & 3.92 & 3.33 & 4.53 & 2.73 & 3.97 & 5.07 & 3.43 & 4.19 & 4.33 & 4.77 \\
\hline $\mathrm{YNb}$ & 3.78 & 5.17 & 4.00 & 3.60 & 3.56 & 44.00 & 5.50 & 3.75 & 5.29 & 3.56 & 4.13 & 3.44 \\
\hline $\mathrm{Ba} / \mathrm{Nb}$ & 23.22 & 45.00 & 35.22 & 26.70 & 35.11 & 270.00 & 22.00 & 36.25 & 58.00 & 19.56 & 24.25 & 23.89 \\
\hline $\mathrm{K} / \mathrm{Ba}$ & 27.41 & 74.41 & 28.28 & 43.22 & 36.25 & 53.81 & 76.10 & 20.32 & 69.11 & 48.58 & 22.25 & 25.10 \\
\hline $\mathrm{K} / \mathrm{Sr}$ & 17.73 & 81.01 & 33.96 & 45.97 & 40.06 & 58.11 & 35.62 & 18.71 & 126.39 & 28.50 & 14.02 & 16.81 \\
\hline $\mathrm{K} / \mathrm{Rb}$ & 520.74 & 490.00 & 332.06 & 576.96 & 498.10 & 1614.20 & 2008.99 & 654.90 & 2550.86 & 777.33 & 863.37 & 490.55 \\
\hline Ti/V & 33.44 & 37.25 & 34.51 & 31.31 & 35.06 & 45.44 & 56.25 & 33.18 & 57.54 & 35.66 & 33.70 & 32.59 \\
\hline \multicolumn{13}{|c|}{$\mathrm{CO}_{2}$ - and $\mathrm{H}_{2} \mathrm{O}$-free normalized values } \\
\hline $\begin{array}{l}\text { Core, Section, } \\
\text { Interval [cm] }\end{array}$ & $\begin{array}{c}33 R-5 \\
109-112\end{array}$ & $\begin{array}{l}34 \mathrm{R}-1 \\
41-44\end{array}$ & $\begin{array}{c}34 \mathrm{R}-2 \\
108-111\end{array}$ & $\begin{array}{l}34 \mathrm{R}-3 \\
66-69\end{array}$ & $\begin{array}{l}34 R-4 \\
39-42\end{array}$ & $\begin{array}{l}34 \mathrm{R}-4 \\
65-68\end{array}$ & $\begin{array}{l}34 R-5 \\
32-35\end{array}$ & $\begin{array}{l}34 R-6 \\
22-25\end{array}$ & $\begin{array}{l}34 \mathrm{R}-6 \\
61-64\end{array}$ & $\begin{array}{c}34 \mathrm{R}-6 \\
93-96\end{array}$ & $\begin{array}{l}35 R-1 \\
88-91\end{array}$ & $\begin{array}{c}35 \mathrm{R}-2 \\
144-147\end{array}$ \\
\hline Rock type & b & br & b & b & b & br & br & b & br & b & b & b \\
\hline $\mathrm{SiO}_{2}$ & 51.95 & 53.34 & 52.78 & 47.07 & 51.98 & 48.84 & 49.85 & 51.80 & 48.73 & 50.22 & 51.99 & 51.91 \\
\hline $\mathrm{TiO}_{2}$ & 1.76 & 1.57 & 1.58 & 1.42 & 1.66 & 1.53 & 1.62 & 1.77 & 1.55 & 1.77 & 1.69 & 1.75 \\
\hline $\mathrm{Al}_{2} \mathrm{O}_{3}$ & 16.05 & 14.45 & 14.75 & 13.34 & 15.08 & 14.08 & 14.99 & 16.19 & 14.29 & 15.60 & 15.71 & 15.99 \\
\hline $\mathrm{Fe}_{2} \mathrm{O}_{3}$ & 8.35 & 8.62 & 4.04 & 8.01 & 6.95 & 11.45 & 12.08 & 5.68 & 12.04 & 7.14 & 6.54 & 8.23 \\
\hline $\mathrm{FeO}$ & 2.71 & 3.67 & 6.81 & 3.91 & 4.27 & 1.01 & 0.90 & 4.00 & 0.62 & 3.18 & 4.30 & 2.98 \\
\hline $\mathrm{MnO}$ & 0.13 & 0.11 & 0.20 & 0.14 & 0.17 & 0.17 & 0.19 & 0.22 & 0.20 & 0.22 & 0.13 & 0.11 \\
\hline $\mathrm{MgO}$ & 6.91 & 5.91 & 6.18 & 5.45 & 6.37 & 5.60 & 6.02 & 7.35 & 5.13 & 6.85 & 6.67 & 6.87 \\
\hline $\mathrm{CaO}$ & 8.16 & 6.70 & 9.61 & 15.89 & 9.03 & 12.46 & 10.14 & 8.95 & 11.36 & 10.72 & 9.39 & 8.26 \\
\hline $\mathrm{Na}_{2} \mathrm{O}$ & 2.94 & 2.74 & 2.62 & 2.92 & 2.71 & 2.69 & 2.66 & 2.93 & 2.09 & 2.83 & 2.73 & 2.93 \\
\hline $\mathrm{K}_{2} \mathrm{O}$ & 0.72 & 2.52 & 1.10 & 1.52 & 1.42 & 1.86 & 1.29 & 0.74 & 3.67 & 1.12 & 0.54 & 0.68 \\
\hline $\mathrm{P}_{2} \mathrm{O}_{5}$ & 0.18 & 0.23 & 0.19 & 0.20 & 0.20 & 0.17 & 0.14 & 0.22 & 0.18 & 0.21 & 0.17 & 0.15 \\
\hline$C_{r}$ & 103 & 83 & 106 & 82 & 100 & 95 & 78 & 95 & 78 & 91 & 97 & 97 \\
\hline Co & 46 & 38 & 42 & 39 & 44 & 42 & 44 & 59 & 36 & 49 & 53 & 46 \\
\hline $\mathrm{Ba}$ & 218 & 281 & 322 & 293 & 325 & 287 & 141 & 300 & 441 & 191 & 201 & 224 \\
\hline $\mathrm{v}$ & 316 & 253 & 275 & 271 & 285 & 202 & 173 & 320 & 162 & 298 & 300 & 322 \\
\hline $\mathrm{Ni}$ & 30 & 29 & 27 & 35 & 32 & 29 & 42 & 31 & 28 & 38 & 32 & 28 \\
\hline $\mathrm{Cu}$ & 30 & 69 & 32 & 83 & 76 & 173 & 67 & 88 & 41 & 157 & 104 & 32 \\
\hline $\mathrm{Zn}_{\mathrm{n}}$ & 96 & 101 & 103 & 109 & 101 & 89 & 130 & 121 & 90 & 113 & 107 & 100 \\
\hline $\mathrm{Rb}$ & 11 & 43 & 27 & 22 & 24 & 10 & 5 & 9 & 12 & 12 & 5 & 11 \\
\hline Sr & 337 & 258 & 268 & 275 & 294 & 266 & 301 & 326 & 241 & 326 & 319 & 335 \\
\hline $\mathrm{Y}$ & 35 & 32 & 37 & 39 & 33 & 47 & 35 & 31 & 40 & 35 & 34 & 32 \\
\hline $\mathrm{Zr}$ & 154 & 136 & 143 & 132 & 149 & 128 & 140 & 157 & 138 & 146 & 148 & 154 \\
\hline $\mathrm{Nb}$ & 9 & 6 & 9 & 11 & 9 & 1 & 6 & 8 & 8 & 10 & 8 & 9 \\
\hline Normalization & 1.04 & 1.04 & 1.02 & 1.10 & 1.03 & 1.06 & 1.07 & 1.04 & 1.09 & 1.09 & 1.04 & 1.04 \\
\hline
\end{tabular}


Table 4. (continued).

\begin{tabular}{|c|c|c|c|c|c|c|c|c|c|c|c|}
\hline \multicolumn{12}{|l|}{ Original values } \\
\hline $\begin{array}{l}\text { Core, } \\
\text { Section, } \\
\text { Interval } \\
\text { [cm] }\end{array}$ & $\begin{array}{l}35 R-3 \\
15-18\end{array}$ & $\begin{array}{l}35 R-3 \\
61-64\end{array}$ & $\begin{array}{l}35 R-3 \\
71-73\end{array}$ & $\begin{array}{c}35 R-4 \\
9-12\end{array}$ & $\begin{array}{l}35 R-4 \\
61-64\end{array}$ & $\begin{array}{c}35 R-4 \\
139-142\end{array}$ & $\underset{6-9}{35 R-6}$ & $\begin{array}{l}36 \mathrm{R}-1 \\
89-92\end{array}$ & $\begin{array}{c}36 \mathrm{R}-2 \\
143-146\end{array}$ & $\begin{array}{l}36 \mathrm{R}-3 \\
37-40\end{array}$ & $\begin{array}{r}36 \mathrm{R}-3 \\
98-101\end{array}$ \\
\hline Rock type & br & br & b & $\mathrm{br}$ & $\mathrm{br}$ & br & b & b & b & b & b \\
\hline $\mathrm{SiO}_{2}$ & 47.00 & 48.50 & 48.20 & 48.40 & 47.70 & 46.00 & 48.90 & 50.10 & 51.50 & 50.80 & 51.10 \\
\hline $\mathrm{TiO}_{2}$ & 1.51 & 1.65 & 1.55 & 1.57 & 1.53 & 1.57 & 1.62 & 1.61 & 1.59 & 1.56 & 1.56 \\
\hline $\mathrm{Al}_{2} \mathrm{O}_{3}$ & 13.92 & 15.92 & 13.94 & 14.39 & 14.07 & 14.47 & 14.37 & 14.33 & 14.36 & 14.34 & 14.22 \\
\hline $\mathrm{Fe}_{2} \mathrm{O}_{3}$ & 10.68 & 9.67 & 7.78 & 9.29 & 8.12 & 10.71 & 6.13 & 6.67 & 3.47 & 3.73 & 3.84 \\
\hline $\mathrm{FeO}$ & 2.09 & 1.99 & 3.79 & 3.12 & 2.03 & 1.77 & 4.48 & 4.43 & 7.78 & 7.40 & 7.68 \\
\hline $\mathrm{MnO}$ & 0.13 & 0.14 & 0.15 & 0.14 & 0.16 & 0.12 & 0.20 & 0.17 & 0.27 & 0.26 & 0.26 \\
\hline $\mathrm{MgO}$ & 6.31 & 5.17 & 5.15 & 5.89 & 5.64 & 4.65 & 6.15 & 6.23 & 6.45 & 6.53 & 6.41 \\
\hline $\mathrm{CaO}$ & 8.37 & 7.06 & 9.88 & 8.62 & 9.55 & 10.17 & 9.69 & 9.02 & 9.78 & 9.73 & 9.71 \\
\hline $\mathrm{Na}_{2} \mathrm{O}$ & 2.24 & 2.47 & 2.55 & 2.49 & 2.67 & 2.57 & 2.54 & 2.56 & 2.50 & 2.48 & 2.43 \\
\hline $\mathrm{K}_{2} \mathrm{O}$ & 1.62 & 1.84 & 2.12 & 1.21 & 1.12 & 1.29 & 0.76 & 0.81 & 0.40 & 0.37 & 0.43 \\
\hline $\mathrm{P}_{2} \mathrm{O}_{5}$ & 0.11 & 0.17 & 0.18 & 0.19 & 0.23 & 0.17 & 0.20 & 0.18 & 0.19 & 0.18 & 0.19 \\
\hline $\mathrm{Cr}$ & 84 & 93 & 98 & 86 & 78 & 85 & 86 & 85 & 84 & 87 & 95 \\
\hline Co & 43 & 39 & 41 & 42 & 34 & 34 & 38 & 37 & 43 & 42 & 39 \\
\hline $\mathrm{Ba}$ & 112 & 221 & 300 & 139 & 220 & 221 & 213 & 498 & 287 & 276 & 281 \\
\hline $\mathrm{v}$ & 231 & 267 & 276 & 250 & 208 & 205 & 276 & 276 & 272 & 272 & 262 \\
\hline $\mathrm{Ni}$ & 43 & 30 & 30 & 34 & 22 & 30 & 27 & 27 & 31 & 32 & 29 \\
\hline $\mathrm{Cu}$ & so & 70 & 35 & 55 & 49 & 46 & 51 & 39 & 42 & 49 & 56 \\
\hline $\mathrm{Zn}$ & 84 & 94 & 103 & 98 & 111 & 96 & 103 & 91 & 110 & 111 & 92 \\
\hline $\mathrm{Rb}$ & 8 & 10 & 33 & 8 & 10 & 7 & 14 & 15 & 6 & 5 & 9 \\
\hline $\mathrm{Sr}$ & 259 & 288 & 254 & 281 & 306 & 281 & 302 & 304 & 287 & 283 & 281 \\
\hline $\mathrm{Y}$ & 23 & 32 & 33 & 39 & 39 & 34 & 37 & 33 & 34 & 34 & 33 \\
\hline $\mathbf{Z}_{x}$ & 127 & 142 & 138 & 136 & 139 & 135 & 142 & 143 & 139 & 135 & 136 \\
\hline $\mathrm{Nb}$ & 8 & 8 & 6 & 7 & 5 & 6 & 8 & 9 & 8 & 8 & 9 \\
\hline $\mathrm{CO}_{2}$ & 4.08 & 1.90 & 1.94 & 1.76 & 4.39 & 3.85 & 1.24 & 0.28 & 0.06 & 0.12 & 0.08 \\
\hline $\mathrm{H}_{2} \mathrm{O}$ & 3.57 & 3.27 & 2.22 & 3.13 & 3.40 & 2.69 & 2.42 & 2.57 & 1.17 & 1.32 & 1.26 \\
\hline Sum & 101.74 & 99.89 & 99.58 & 100.32 & 100.72 & 100.15 & 98.83 & 99.11 & 99.66 & 98.96 & 99.29 \\
\hline $\mathrm{Fe}_{2} \mathrm{O}_{3} / \mathrm{FeO}$ & 5.12 & 4.85 & 2.05 & 2.97 & 4.01 & 6.05 & 1.37 & 1.50 & 0.45 & 0.50 & 0.50 \\
\hline $\mathrm{Z} \times \mathrm{Nb}$ & 15.88 & 17.75 & 23.00 & 19.43 & 27.80 & 22.50 & 17.75 & 15.89 & 17.38 & 16.88 & 15.11 \\
\hline $\mathrm{Ti} / \mathrm{Zr}$ & 71.28 & 69.66 & 67.34 & 69.21 & 65.99 & 69.72 & 68.39 & 67.50 & 68.58 & 69.28 & 68.77 \\
\hline $\mathrm{Z} / \mathrm{Y}$ & 5.52 & 4.44 & 4.18 & 3.49 & 3.56 & 3.97 & 3.84 & 4.33 & 4.09 & 3.97 & 4.12 \\
\hline $\mathrm{YNb}$ & 2.88 & 4.00 & 5.50 & 5.57 & 7.80 & 5.67 & 4.63 & 3.67 & 4.25 & 4.25 & 3.67 \\
\hline $\mathrm{Ba} / \mathrm{Nb}$ & 14.00 & 27.63 & 50.00 & 19.86 & 44.00 & 36.83 & 26.63 & 55.33 & 35.88 & 34.50 & 31.22 \\
\hline $\mathrm{K} / \mathrm{Ba}$ & 120.08 & 69.12 & 58.66 & 72.27 & 42.26 & 48.46 & 29.62 & 13.50 & 11.57 & 11.13 & 12.70 \\
\hline $\mathrm{K} / \mathrm{Sr}$ & 51.93 & 53.04 & 69.29 & 35.75 & 30.38 & 38.11 & 20.89 & 22.12 & 11.57 & 10.85 & 12.70 \\
\hline $\mathrm{K} / \mathrm{Rb}$ & 1681.07 & 1527.49 & 533.31 & 1255.62 & 929.78 & 1529.87 & 450.66 & 448.29 & 553.44 & 614.32 & 396.63 \\
\hline $\mathrm{Ti} / \mathrm{N}$ & 39.19 & 37.05 & 33.67 & 37.65 & 44.10 & 45.91 & 35.19 & 34.97 & 35.04 & 34.38 & 35.70 \\
\hline \multicolumn{12}{|c|}{$\mathrm{CO}_{2}$ - and $\mathrm{H}_{2} \mathrm{O}$-free normalized values } \\
\hline & 35R-3 & 35R-3 & 35R-3 & $35 \mathrm{R}-4$ & $35 \mathrm{R}-4$ & $35 \mathrm{R}-4$ & $35 \mathrm{R}-6$ & $36 \mathrm{R}-1$ & $36 \mathrm{R}-2$ & 36R-3 & $36 \mathrm{R}-3$ \\
\hline Core, Section & $15-18$ & $61-64$ & $71-73$ & $9-12$ & $61-64$ & $139-142$ & $6-9$ & $89-92$ & $143-146$ & $37-40$ & 98-101 \\
\hline Rock type & br & br & b & $\mathrm{br}$ & br & br & b & b & b & b & b \\
\hline $\mathrm{SiO}_{2}$ & 49.95 & 51.20 & 50.51 & 50.72 & 51.33 & 49.14 & 51.38 & 52.05 & 52.32 & 52.09 & 52.17 \\
\hline $\mathrm{TiO}_{2}$ & 1.60 & 1.74 & 1.62 & 1.65 & 1.65 & 1.68 & 1.70 & 1.67 & 1.62 & 1.60 & 1.59 \\
\hline $\mathrm{Al}_{2} \mathrm{O}_{3}$ & 14.80 & 16.81 & 14.61 & 15.08 & 15.14 & 15.46 & 15.10 & 14.89 & 14.59 & 14.70 & 14.52 \\
\hline $\mathrm{Fe}_{2} \mathrm{O}_{3}$ & 11.35 & 10.21 & 8.15 & 9.73 & 8.74 & 11.44 & 6.44 & 6.93 & 3.53 & 3.83 & 3.92 \\
\hline $\mathrm{FeO}$ & 2.22 & 2.11 & 3.97 & 3.27 & 2.18 & 1.89 & 4.71 & 4.60 & 7.90 & 7.59 & 7.84 \\
\hline $\mathrm{MnO}$ & 0.14 & 0.15 & 0.16 & 0.15 & 0.17 & 0.13 & 0.21 & 0.18 & 0.27 & 0.27 & 0.27 \\
\hline $\mathrm{MgO}$ & 6.71 & 5.46 & 5.40 & 6.17 & 6.07 & 4.97 & 6.46 & 6.47 & 6.55 & 6.70 & 6.54 \\
\hline $\mathrm{CaO}$ & 8.90 & 7.45 & 10.35 & 9.03 & 10.28 & 10.86 & 10.18 & 9.37 & 9.94 & 9.98 & 9.91 \\
\hline $\mathrm{Na}_{2} \mathrm{O}$ & 2.38 & 2.61 & 2.67 & 2.61 & 2.87 & 2.75 & 2.67 & 2.66 & 2.54 & 2.54 & 2.48 \\
\hline $\mathrm{K}_{2} \mathrm{O}$ & 1.72 & 1.94 & 2.22 & 1.27 & 1.21 & 1.38 & 0.80 & 0.84 & 0.41 & 0.38 & 0.44 \\
\hline $\mathrm{P}_{2} \mathrm{O}_{5}$ & 0.12 & 0.18 & 0.19 & 0.20 & 0.25 & 0.18 & 0.21 & 0.19 & 0.19 & 0.18 & 0.19 \\
\hline $\mathrm{Cr}$ & 89 & 98 & 103 & 90 & 84 & 91 & 90 & 88 & 85 & 89 & 97 \\
\hline Co & 46 & 41 & 43 & 44 & 37 & 36 & 40 & 38 & 44 & 43 & 40 \\
\hline $\mathrm{Ba}$ & 119 & 233 & 314 & 146 & 237 & 236 & 224 & 517 & 292 & 283 & 287 \\
\hline$v$ & 246 & 282 & 289 & 262 & 224 & 219 & 290 & 287 & 276 & 279 & 267 \\
\hline $\mathrm{Ni}$ & 46 & 32 & 31 & 36 & 24 & 32 & 28 & 28 & 31 & 33 & 30 \\
\hline $\mathrm{Cu}$ & 53 & 74 & 37 & 58 & 53 & 49 & 54 & 41 & 43 & 50 & 57 \\
\hline $\mathrm{Zn}$ & 89 & 99 & 108 & 103 & 119 & 103 & 108 & 95 & 112 & 114 & 94 \\
\hline $\mathrm{Rb}$ & 9 & 11 & 35 & 8 & 11 & 7 & 15 & 16 & 6 & 5 & 9 \\
\hline Sr & 275 & 304 & 266 & 294 & 329 & 300 & 317 & 316 & 292 & 290 & 287 \\
\hline $\mathbf{Y}$ & 24 & 34 & 35 & 41 & 42 & 36 & 39 & 34 & 35 & 35 & 34 \\
\hline $\mathbf{z}$ & 135 & 150 & 145 & 143 & 150 & 144 & 149 & 149 & 141 & 138 & 139 \\
\hline $\mathrm{Nb}$ & 9 & 8 & 6 & 7 & 5 & 6 & 8 & 9 & 8 & 8 & 9 \\
\hline $\begin{array}{l}\text { Normalization } \\
\text { factor }\end{array}$ & 1.06 & 1.06 & 1.05 & 1.05 & 1.08 & 1.07 & 1.05 & 1.04 & 1.02 & 1.03 & 1.02 \\
\hline
\end{tabular}


K. W. MEHL, P. R. BITSCHENE, H.-U. SCHMINCKE, J. HERTOGE
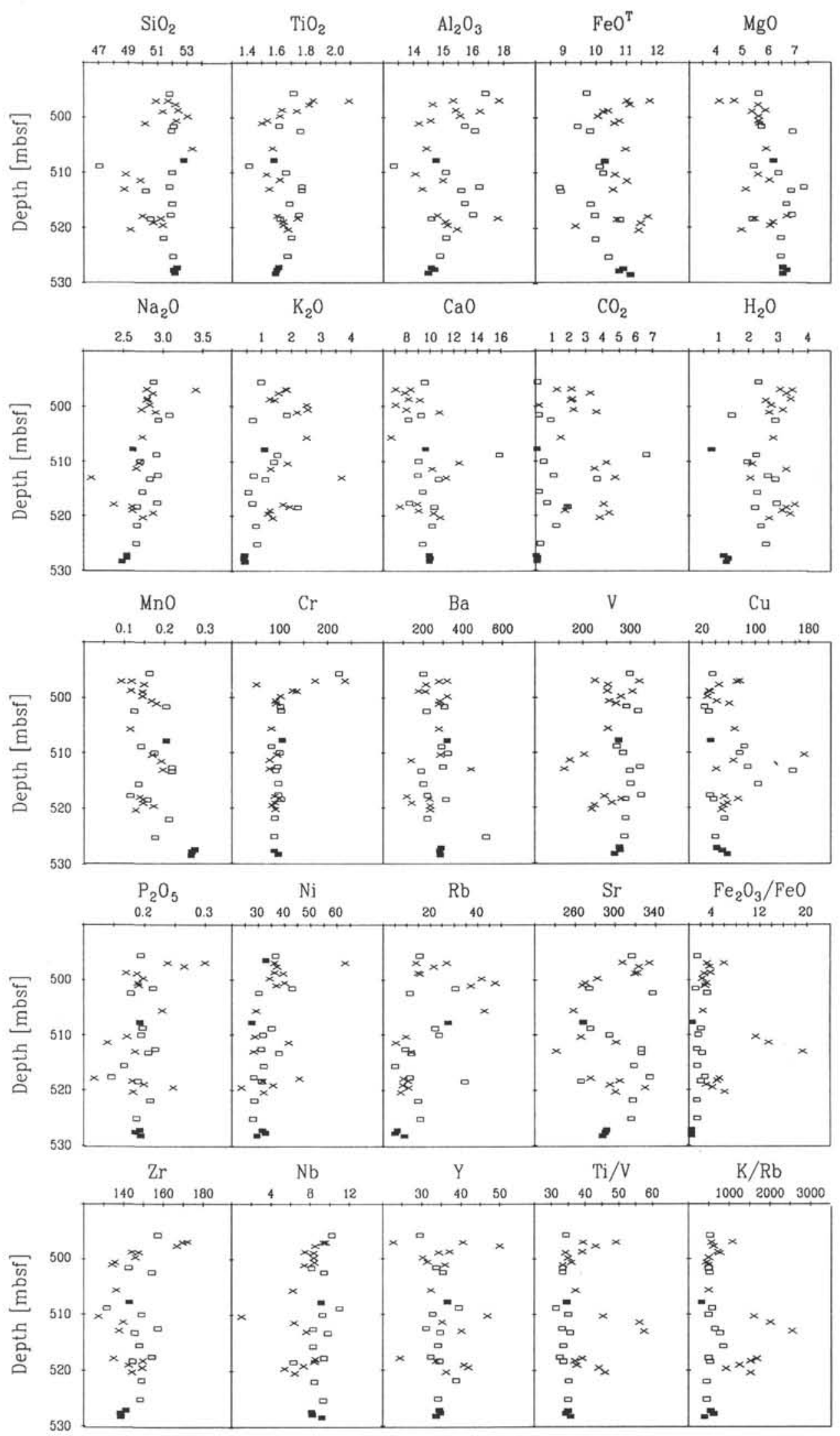

Figure 8. Variation of major and trace element concentrations and element ratios of Unit VIII samples. Oxides in wt\%, elements in ppm. Crosses represent breccias, quadrangles represent basalts, and filled quadrangles represent the four freshest basalts. 
A
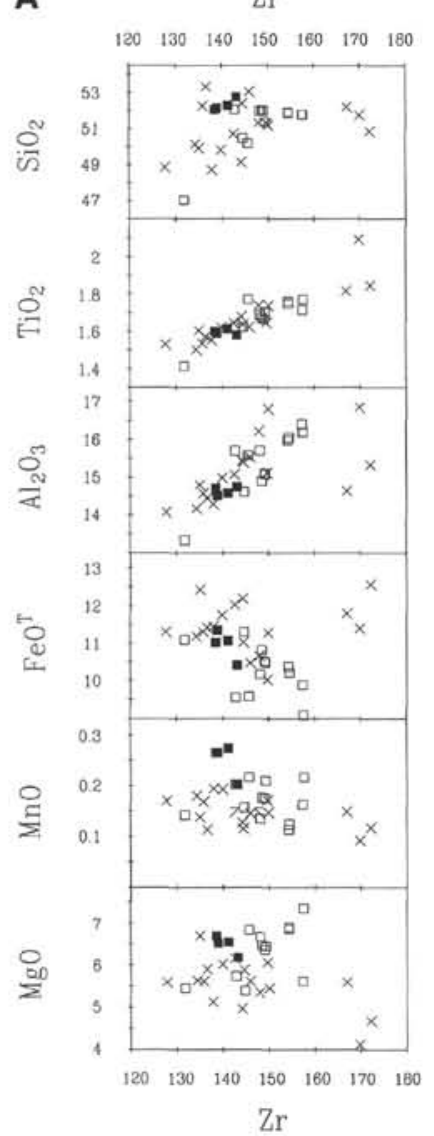

$\mathrm{Zr}$

B

$\mathrm{Zr}$

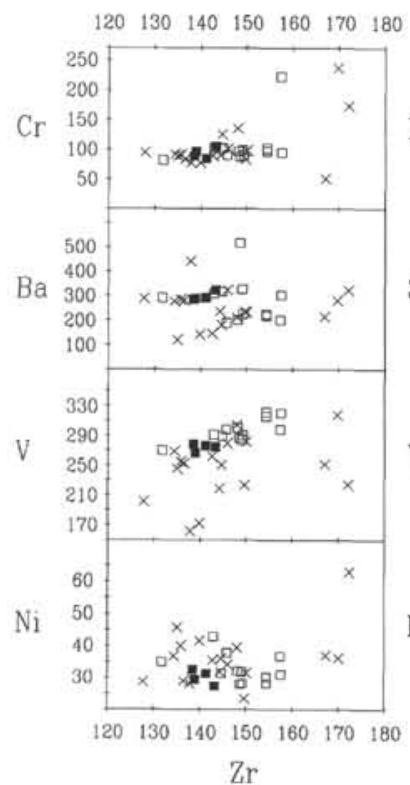

$\mathrm{Zr}$

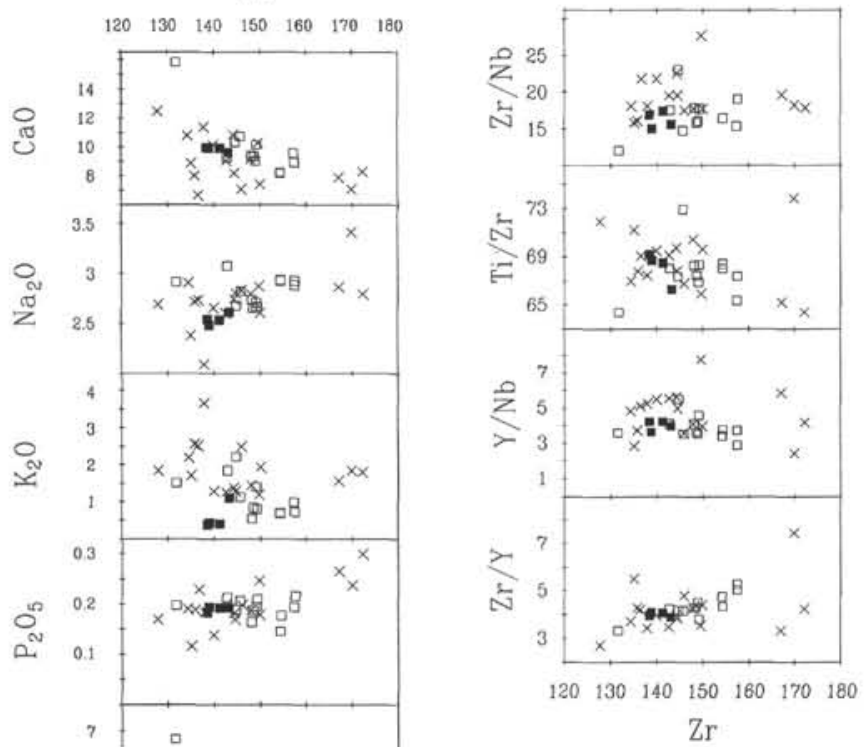

$\mathrm{Zr}$

$\begin{array}{lllllll}120 & 130 & 140 & 150 & 160 & 170 & 180\end{array}$

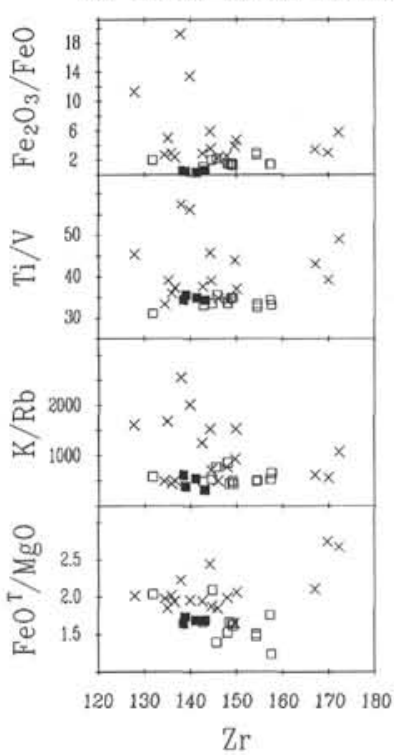

Figure 9. (continued).

seawater, whereas saponite forms under nonoxidative conditions and a high $\mathrm{pH}(\geq 8.5)$ from modified percolating seawater (Andrews et al., 1977; Seyfried et al., 1978; Andrews, 1979). Both may form simultaneously reflecting gradual and systematic small-scale oscillation of $\mathrm{Eh} / \mathrm{pH}$ conditions, e.g., from a vein into the adjacent basalt (Andrews, 1979). Andrews (1980) reports saponite being formed in oxidative as well as in nonoxidative environments differing in their compositions: $\mathrm{Mg}$-rich, relative $\mathrm{Fe}$-poor saponite occurring in oxidative environments and Fe-rich saponite with lower $\mathrm{Mg}$ contents in nonoxidative environments.

$\mathrm{Ca}, \mathrm{Fe}$, and $\mathrm{Si}$, needed to form sheet silicates and calcite, are thought to be released from the basalt during alteration (Seyfried et al., 1978; Staudigel and Hart, 1983). $\mathrm{Mg}, \mathrm{Si}$, and $\mathrm{Sr}$ may be released during alteration of interstitial glass, but also may be gained from sea water (Staudigel et al., 1981). K, $\mathrm{Rb}, \mathrm{H}_{2} \mathrm{O}$, and $\mathrm{CO}_{2}$ are removed from seawater (Seyfried et al., 1978; Staudigel and Hart, 1983) during formation of the alteration mineral assemblage.

In Figure 12 the freshest basalt sample is used as a normalizer against the most altered samples. The resulting spidergram allows a quantitative estimate of the alteration when roughly identical primary compositions for both the fresh basalt and the altered samples are assumed. This is inferred from ratios near unity for immobile elements such as $\mathrm{Zr}, \mathrm{Ti}, \mathrm{Nb}, \mathrm{Cr}$, and others. Altered basalts show a ten-fold increase in $\mathrm{CO}_{2}$, a two- to three-fold increase in $\mathrm{K}_{2} \mathrm{O}, \mathrm{Rb}, \mathrm{H}_{2} \mathrm{O}$, and a high degree of oxidation $\left(\mathrm{ox}=\mathrm{Fe}_{2} \mathrm{O}_{3} / \mathrm{FeO}\right)$, whereas $\mathrm{P}_{2} \mathrm{O}_{5}, \mathrm{Ba}$, and $\mathrm{CaO}$ show a $10 \%-20 \%$ decrease (Fig. 12). The volcaniclastic samples display the same pattern but with nearly ninety-fold increase in $\mathrm{CO}_{2}$, up to ten-fold increase in $\mathrm{K}_{2} \mathrm{O}$, and up to four-fold increase in $\mathrm{Rb}$, and $\mathrm{H}_{2} \mathrm{O}$. For $\mathrm{P}_{2} \mathrm{O}_{5}, \mathrm{~V}, \mathrm{Y}, \mathrm{Ba}$, and $\mathrm{MgO}$ a $20 \%-40 \%$ loss can be estimated.

Assuming a primary T-MORB composition and total element removal from seawater $(\mathrm{K}, 390 \mathrm{ppm}$; Rb, $0.12 \mathrm{ppm}$; Ba, $0.02 \mathrm{ppm}$; Manson and Moore, 1982), water/rock ratios of about 10 for $\mathrm{K}_{2} \mathrm{O}$, and 85 for $\mathrm{Rb}$, are at least required for the basalts. For the breccias water/rock ratios of 30 and 185 are required for $\mathrm{K}_{2} \mathrm{O}$ and $\mathrm{Rb}$, respectively. The contribution of seawater $\mathrm{Ba}$ to the enriched $\mathrm{Ba}$ concentrations is negligible if reasonable water/rock ratios are considered. $\mathrm{Ba}$ may therefore be either derived from upwelling hydrothermal solutions (Mottl and Holland, 1978; Seyfried and Bischoff, 1979), for

Figure 9. A. Variation of major elements of Unit VIII samples vs. Zr. Oxides in wt\%. B. Variation of trace elements of Unit VIII samples vs. Zr. Elements in ppm. C. Variation of element ratios of Unit VIII samples vs. Zr. Symbols as in Figure 8. 

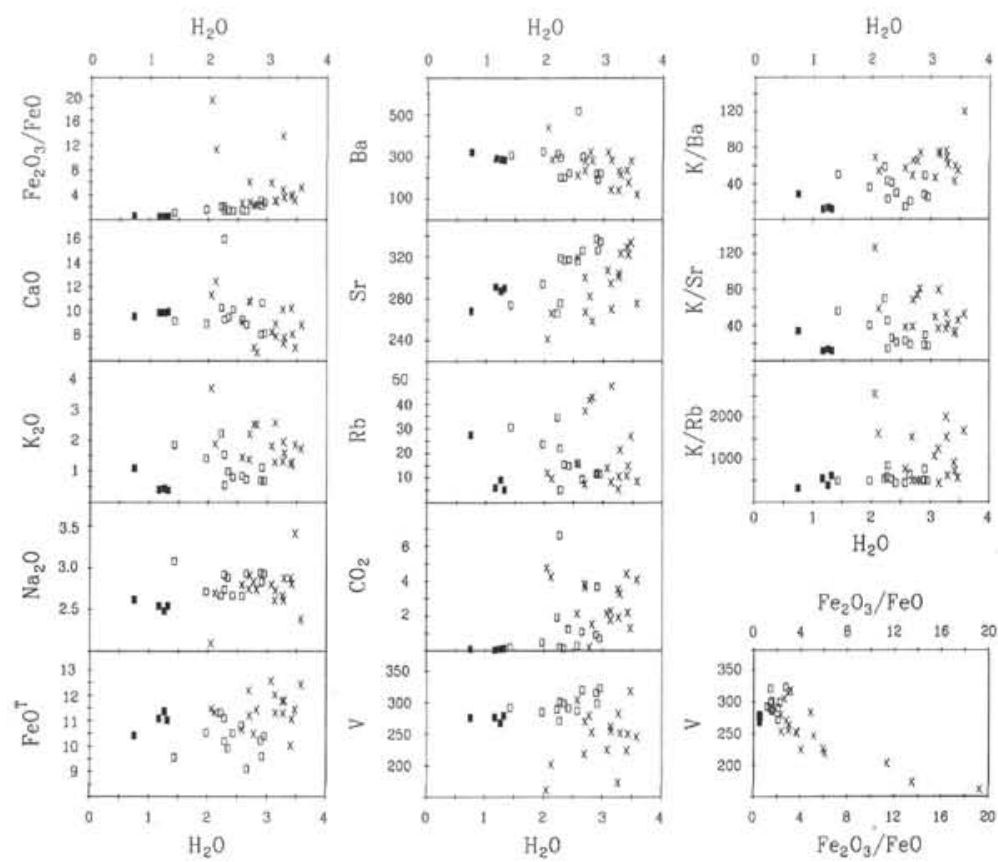

Figure 10. Variation of mobile elements and element ratios of Unit VIII samples vs. $\mathrm{H}_{2} \mathrm{O}$ and $\mathrm{V}$ vs. $\mathrm{Fe}_{2} \mathrm{O}_{3} / \mathrm{FeO}$. Oxides in wt\%, elements in ppm. Symbols as in Figure 8.

Table 5. CIPW norms of the freshest samples. Assumed $\mathrm{Fe}_{2} \mathrm{O}_{3}=2 \%$.

\begin{tabular}{lcccc}
\hline & $\begin{array}{c}119-738-34 R-2 \\
108-111 \mathrm{~cm}\end{array}$ & $\begin{array}{c}119-738-36 \mathrm{R}-2 \\
143-146 \mathrm{~cm}\end{array}$ & $\begin{array}{c}119-738-36 \mathrm{R}-3 \\
37-40 \mathrm{~cm}\end{array}$ & $\begin{array}{c}119-738-36 \mathrm{R}-3 \\
98-101 \mathrm{~cm}\end{array}$ \\
\hline Quartz & 3.31 & 4.05 & 3.68 & 3.95 \\
Orthoclase & 6.50 & 2.42 & 2.25 & 2.60 \\
Albite & 22.25 & 21.58 & 21.58 & 21.07 \\
Anorthite & 25.30 & 27.24 & 27.63 & 27.23 \\
Diopside & 17.10 & 16.89 & 16.46 & 16.73 \\
Hypersthene & 18.97 & 21.22 & 21.70 & 21.80 \\
Magnetite & 2.96 & 2.96 & 2.97 & 2.97 \\
Ilmenite & 3.02 & 3.08 & 3.04 & 3.04 \\
Apatite & 0.44 & 0.44 & 0.42 & 0.44 \\
Calcite & 0.16 & 0.14 & 0.27 & 0.18 \\
\hline
\end{tabular}

which there is no indication in our basalts, or be a primary feature of the basalt magma, which is assumed here. $\mathrm{V}$ is leached and removed from the breccias ( $\mathrm{Ti}$ - magnetite therein) probably because of higher water/rock ratios (Table 8.)

The significantly higher alteration of breccias compared to lava flows is attributed to higher water/rock ratios as previously discussed due to higher permeabilities of the breccias (porosities are about $35 \%$; Barron, Larsen, et al., 1989) and has previously been reported, e.g., from Icelandic basalts (Mehegan and Robinson, 1982; Viereck et al., 1982).

The chemical and mineralogical changes of the basement rocks are mainly due to low-temperature alteration. The gains of $\mathrm{K}_{2} \mathrm{O}, \mathrm{Rb}$, and $\mathrm{H}_{2} \mathrm{O}$, and the loss of $\mathrm{CaO}$ as well as the alteration of plagioclase and clinopyroxene to sheet silicates indicate low-temperature alteration. The occurrence of celadonite and saponite limits the alteration temperature to about $170^{\circ} \mathrm{C}$ (Seyfried et al., 1978; Seyfried and Bischoff, 1979; Andrews, 1979, 1980). This is in agreement with the whole secondary mineral assemblage (Fig. 13) and the lack of secondary high-temperature phases such as sphene, chlorite, or specific zeolite.

The occurrence of $\mathrm{Fe}$-oxides and Fe-hydroxides, celadonite, and green and brown saponite clearly reflects changing $\mathrm{Eh} / \mathrm{pH}$ conditions during alteration.

The high oxidation and abundant calcite may be due to subsequent final cold seawater alteration. Cold seawater alteration may not have

Table 6. Incompatible trace element ratios of Mesozoic N/T/P-MORB, OIB, and CFB from the Southern Hemisphere.

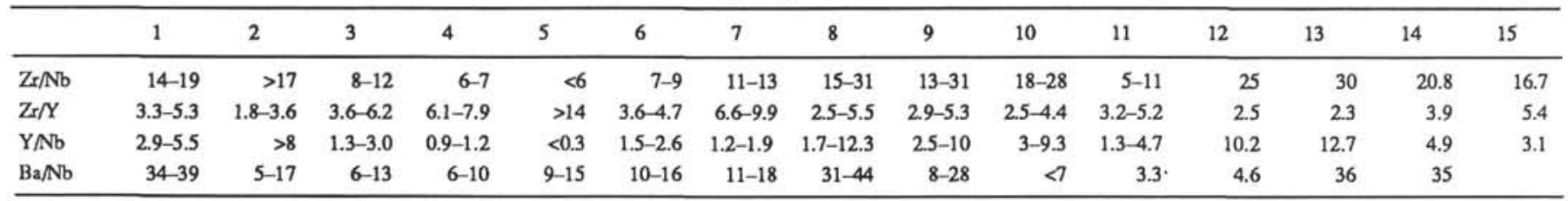

1= T-MORB from ODP Leg 119, Site 738; 2 = N-MORB from SWIR (LeRoex et al., 1983); 3 = T-MORB from SWIR (LeRoex et al., 1983); 4 = E/P-MORB from SWIR (LeRoex et al., 1983); $5=$ OIB from ODP Leg 120, Site 748 (Bitschene et al., in prep); $6=$ tholeiite from Kerguelen Island (Storey et al., 1988 ); $7=$ Na-alkali basalt from Kerguelen Island (Storey et al., 1988); 8 = tholeiites from SEIR (Dosso et al., 1988); 9 = low-Ti CFB from the Parana Basin/South America (Bitschene, 1987; Mantovani et al., 1988); 10 = CFB from Rajmahal Trap/India (Mahoney et al., 1983); 11 = MORB from NER (Mahoney et al., 1983); 12 = N2-MORB (Viereck et al., 1989); 13 = N1-MORB (Viereck et al., 1989); 14 = Central Karroo low-Mg CFB from Lesotho (Erlank, 1984); 15 = CFB from the Etendeka-Formation/Namibia (Duncan et al., 1984). 


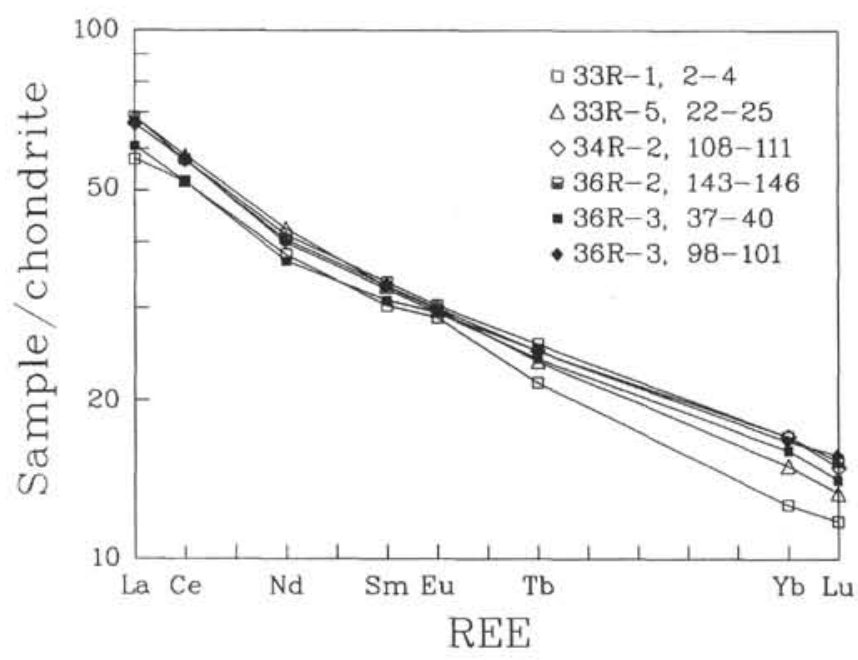

Figure 11. Variation of chondrite-normalized REE concentrations of selected Hole 738C samples. Normalization values from Sun and McDonough (1989).

been very effective as the typical replacement of groundmass-plagioclase by $\mathrm{K}$-feldspar, and the resulting chemical changes were not observed. It may have been terminated by early sealing of the channelways.

\section{PETROGENESIS}

The incompatible and immobile trace elements $\mathrm{Zr}, \mathrm{Nb}, \mathrm{Y}, \mathrm{Ta}$, and $\mathrm{Ti}$, and the REE and their ratios are considered to represent original magma and source characteristics not affected by secondary alteration processes. These elements are used for characterization of the basaltic magmas and explanation of differences within the complete suite of basalts and breccias. $\mathrm{Rb}, \mathrm{K}, \mathrm{Ba}$, and $\mathrm{Th}$ have to be treated separately,

Table 7. REE and trace element concentrations analyzed by INAA of selected Hole $738 \mathrm{C}$ samples. $\mathrm{Na}$ and $\mathrm{Fe}$ in wt \%, other elements in ppm. cn $=$ chondrite normalized.

\begin{tabular}{lcccccc}
\hline Section, & $33 R-1$ & $33 R-5$ & $34 R-2$ & $36 R-2$ & $36 R-3$ & $36 R-3$ \\
Interval & $2-4$ & $22-25$ & $108-111$ & $143-146$ & $37-40$ & $98-101$ \\
\hline $\mathrm{Na}$ & 2.19 & 2.22 & 2.16 & 2.06 & 2.03 & 2.04 \\
$\mathrm{Sc}$ & 36.1 & 36.7 & 35.2 & 35.2 & 35.1 & 35.0 \\
$\mathrm{Cr}$ & 229 & 104 & 96 & 84 & 87 & 84 \\
$\mathrm{Fe}$ & 7.45 & 7.35 & 8.12 & 8.59 & 8.50 & 8.87 \\
$\mathrm{Co}$ & 50.0 & 45.3 & 45.5 & 45.9 & 46.5 & 43.6 \\
$\mathrm{Rb}$ & 23 & 38 & 22 & $<8$ & $<8$ & $<11$ \\
$\mathrm{Sr}$ & 360 & 292 & 269 & 321 & 299 & 262 \\
$\mathrm{Ba}$ & 164 & 352 & 280 & 266 & 252 & 229 \\
& & & & & & \\
$\mathrm{La}$ & 13.6 & 16.3 & 15.8 & 16.3 & 14.4 & 15.8 \\
$\mathrm{Ce}$ & 31.9 & 35.7 & 35.0 & 35.1 & 31.9 & 35.2 \\
$\mathrm{Nd}$ & 17.7 & 19.8 & 18.9 & 19.3 & 17.2 & 18.6 \\
$\mathrm{Sm}$ & 4.64 & 5.05 & 5.06 & 5.14 & 4.75 & 5.00 \\
$\mathrm{Eu}$ & 1.67 & 1.73 & 1.74 & 1.76 & 1.71 & 1.71 \\
$\mathrm{~Tb}$ & .81 & .89 & .93 & .96 & .90 & .93 \\
$\mathrm{Yb}$ & 2.16 & 2.56 & 2.91 & 2.91 & 2.74 & 2.85 \\
$\mathrm{Lu}$ & .30 & .34 & .38 & .39 & .36 & .40 \\
& & & & & & \\
$\mathrm{Hf}$ & 4.1 & 3.6 & 3.6 & 3.7 & 3.6 & 3.5 \\
$\mathrm{Ta}$ & .64 & .55 & .54 & .55 & .55 & .53 \\
$\mathrm{Th}$ & 2.7 & 2.1 & 2.2 & 2.2 & 2.2 & 2.2 \\
$\mathrm{U}$ & $<.15$ & .37 & .22 & .20 & $<.15$ & $<.15$ \\
& & & & & & \\
(Ce/Yb) cn & 4.10 & 3.87 & 3.34 & 3.35 & 3.23 & 3.43 \\
\hline & & & & & &
\end{tabular}

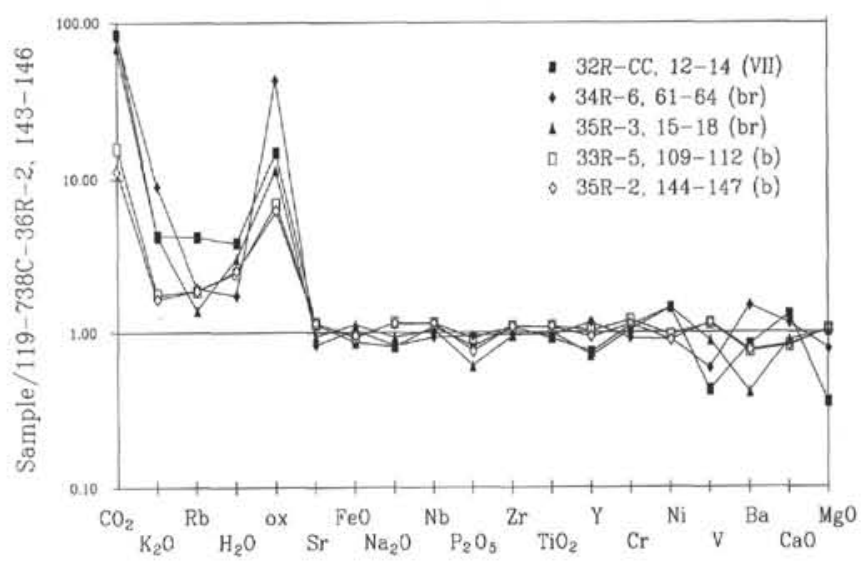

Figure 12. Spidergram showing alteration of selected Hole $738 \mathrm{C}$ samples. Concentrations are normalized against the freshest sample. VII = Unit VII, b $=$ basalt of Unit VIII, $b r=$ breccia of Unit VIII, $o x=\mathrm{Fe}_{2} \mathrm{O}_{3} / \mathrm{FeO}$.

because alteration may lead to precipitation or mobilization of these elements. As shown in the alteration section, high $\mathrm{Ba}$ (and low $\mathrm{Rb}$ and $\mathrm{K}_{2} \mathrm{O}$ ) concentrations of the least altered samples are primary features of the magma. These samples (see norms in Table 5) are used to constrain source regions and to make comparisons with other tholeiitic basalts.

\section{Magma Characteristics and Comparison with Other Mesozoic Southern Hemisphere Basalts}

The freshest Site 738 basalts have Rb concentrations between 5 and $9 \mathrm{ppm}$, higher than $\mathrm{Rb}$ concentrations from the freshest N-MORB glasses (0.4-4 ppm; LeRoex, 1987) or N1-MORB (1.4 ppm) and N2-MORB (1.8 ppm; Viereck et al., 1989), but equivalent to average T-MORB from the southern oceans (5.9 ppm; LeRoex, 1987). $\mathrm{K}_{2} \mathrm{O}$ concentrations are between $0.37 \%$ and $0.42 \%$, which is less than average southern ocean T-MORB (0.56\%; LeRoex, 1987), and more than N-MORB (<0.11\%; LeRoex 1987; Viereck et al. 1989). Ba concentrations for average Southern Ocean T-MORB (90 ppm;

Table 8. Alteration of basement rocks at Hole 738C.

\begin{tabular}{lrcrrc}
\hline & \multicolumn{1}{c}{1} & $2 \mathrm{a} / \mathrm{b}$ & \multicolumn{1}{c}{3} & \multicolumn{1}{c}{4} & 5 \\
\hline $\mathrm{SiO}_{2}$ & 52.40 & $0.997 / 1.011$ & 52.26 & 52.98 & - \\
$\mathrm{MgO}$ & 6.50 & $0.995 / 0.889$ & 6.47 & 5.78 & - \\
$\mathrm{FeO}$ & 10.99 & $0.954 / 1.082$ & 10.49 & 11.89 & 8.67 \\
$\mathrm{~K}_{2} \mathrm{O}$ & 0.58 & $1.983 / 3.379$ & 1.15 & 1.96 & 0.56 \\
$\mathrm{Na}_{2} \mathrm{O}$ & 2.55 & $1.133 / 1.114$ & 2.89 & 2.84 & - \\
$\mathrm{CaO}_{\text {corr }}$ & 9.76 & $0.829 / 0.561$ & 8.09 & 5.48 & - \\
$\mathrm{CO} 2$ & 0.08 & $18.12 / 34.62$ & 1.45 & 2.77 & - \\
$\mathrm{H} 2 \mathrm{O}$ & 1.13 & $2.133 / 2.637$ & 2.41 & 2.98 & - \\
$\mathrm{Rb}$ & $6.8 *$ & $2.579 / 2.981$ & 17.54 & 20.27 & 5.9 \\
$\mathrm{Sr}$ & 284.45 & $1.107 / 1.073$ & 314.80 & 305.19 & 286 \\
$\mathrm{Ba}$ & 296.12 & $0.953 / 0.870$ & 282.10 & 257.74 & 90 \\
$\mathrm{ox}$ & 0.31 & $1.968 / 2.548$ & 0.61 & 0.79 & - \\
$\mathrm{K} / \mathrm{Rb}$ & 474.11 & $1.204 / 2.329$ & 570.99 & 1104.34 & 788 \\
$\mathrm{~K} / \mathrm{Sr}$ & 17.27 & $1.814 / 3.191$ & 31.33 & 55.11 & 16.2 \\
$\mathrm{~K} / \mathrm{Ba}$ & 15.92 & $2.174 / 4.094$ & 34.62 & 65.19 & 51.7 \\
$\mathrm{Ti} / \mathrm{V}$ & 34.91 & $0.970 / 1.196$ & 33.88 & 41.76 & 44.6 \\
\hline
\end{tabular}

$1=$ mean of four freshest basalts (Samples 119-738C-34R-2, 108$111 \mathrm{~cm} ;-36 \mathrm{R}-2,143-146 \mathrm{~cm} ;-36 \mathrm{R}-3,37-40 \mathrm{~cm} ;-36 \mathrm{R}-3,98-101$ $\mathrm{cm}) ; 2$ = alteration factor: (a) mean of basalts/mean of four freshest basalts and (b) mean of breccias/mean of four freshest basalts; 3 = mean of basalts except the four freshest basalts; 4 = mean of breccias; $5=$ T-MORB (LeRoex 1987); * = only three freshest basalts 


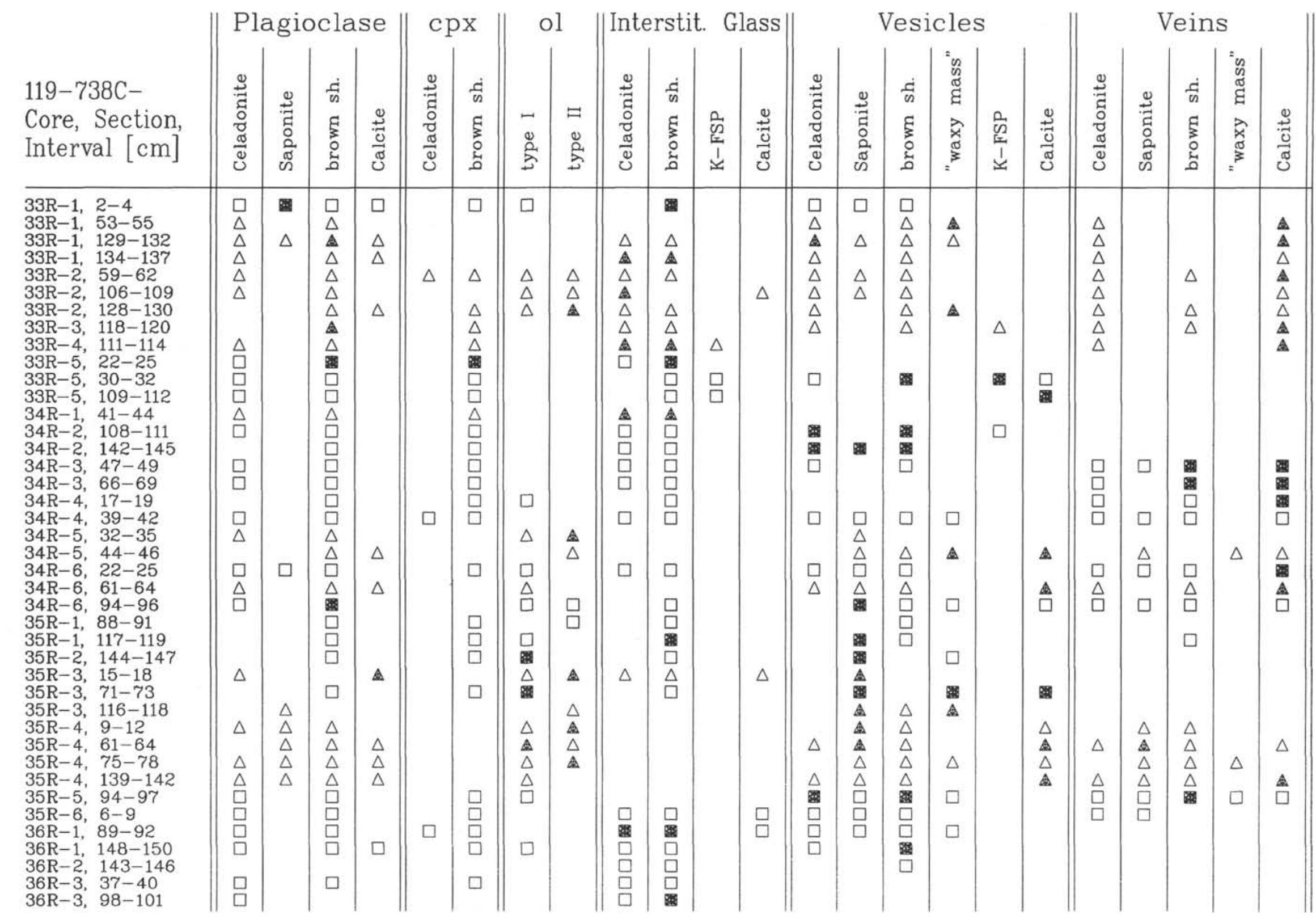




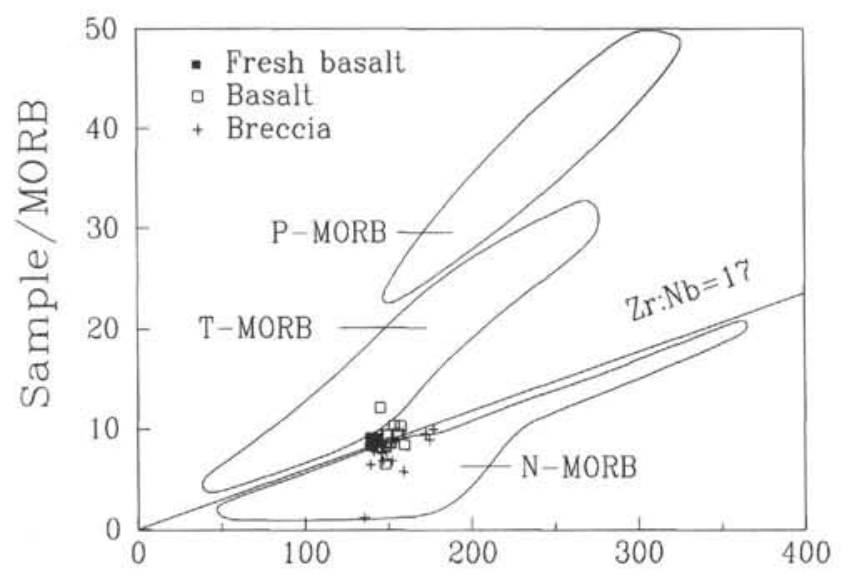

Figure 14. Variation of $\mathrm{Nb}$ vs. Zr. Reference fields for Southern Ocean MORB-types from LeRoex (1987).

LeRoex, 1987) and P-MORB (163 ppm; LeRoex, 1987) are significantly lower than in fresh Site 738 basalts $(290 \pm 10 \mathrm{ppm})$. The high $\mathrm{Ba}$ and Th concentrations are considered to be primary features, as well as the low $\mathrm{Nb}$ and $\mathrm{Ta}$ concentrations.

Tholeiitic MORB are customarily divided into LREE depleted N-MORB, and LREE enriched E-MORB (e.g., Sun et al., 1979; Viereck et al., 1989). Compared to N-MORB types (e.g., Klein and Langmuir, 1988; Viereck et al., 1989), the Site 738 basalts have distinctly higher LREE/HREE ratios ( $>3$ for Site 738 basalts; $<1$ for $\mathrm{N}$-MORB types) and higher HFSE concentrations, which confirms their enriched (E-MORB) character. E-MORB are furthermore divided into transitional basalts (T-MORB with relatively small LREE enrichments and $\mathrm{Zr} / \mathrm{Nb}$ ratios between 10 and 17 ), and plume-type MORB (P-MORB with low $\mathrm{Zr} / \mathrm{Nb}$ ratios between 4 and 7 and high LREE/HREE ${ }_{\text {cn }}$ ratios; e.g., LeRoex 1987). Ocean island basalts (OIB) have P-MORB trace element ratios and isotope characteristics.

LREE/HREE (3.2-4.1) and $\mathrm{Zr} / \mathrm{Nb}$ (14-19, Fig. 14) ratios of Site 738 basalts are intermediate between N-MORB and P/T-MORB (Tables 7 and 9, and Figs. 14 and 15). The observed trace element characteristics (Fig. 15, Table 9) have a close match with T-MORB from the Southwest Indian Ridge, and even a better match with continental flood basalts. $\mathrm{Ba}$ concentrations and $\mathrm{Ba} / \mathrm{Nb}$ ratios of Site 738 basalts are particularly useful in demonstrating the close relationship to Mesozoic continental flood basalts.

\section{Magma Evolution}

Site 738 basalt flows have low $\mathrm{Ni}(32+11 /-5 \mathrm{ppm})$ and moderate $\mathrm{Cr}(95+8 /-13 \mathrm{ppm}$, except for the topmost sample) concentrations. The petrogenetic implications would be olivine, $\mathrm{Cr}$-clinopyroxene and minor $\mathrm{Cr}$-spinel fractionation leading to the evolved basaltic composition that is also indicated by $\mathrm{MgO}<7.35 \%$. Fractionation of about $8 \%$ of olivine with $\mathrm{Kd}$ for $\mathrm{Ni}$ of about 15 would lower an initial $\mathrm{Ni}$ concentration of $250 \mathrm{ppm}$ (primitive MORB) to the observed 30-40 $\mathrm{ppm}$ level. Cr-enrichment and respective clinopyroxene accumulation of the topmost samples may indicate the final eruption pulse, when clinopyroxene-enriched "leftovers" are tapped and erupted. Admixture of only $1 \%-2 \%$ of $\mathrm{Cr}$ - (and $\mathrm{Mg}$-) rich clinopyroxene ( $\mathrm{Cr}$ concentration about $10,000 \mathrm{ppm}$ ) to the basaltic magma is capable of raising average bulk-rock $\mathrm{Cr}$ concentrations from $95 \mathrm{ppm}$ to the observed 240 ppm level. Such $\mathrm{Cr}$ - and $\mathrm{Mg}$-rich clinopyroxene phenocrysts with up to $1.20 \% \mathrm{Cr}_{2} \mathrm{O}_{3}$ were found within the topmost sample.

Considering the whole data set, some variations in immobile element concentrations are observed, which do not comply with simple fractional crystallization:
1. The chemically more evolved samples $(\mathrm{Zr}>150 \mathrm{ppm})$ display the highest compatible element abundances $(\mathrm{Cr}>95 \mathrm{ppm} ; \mathrm{MgO}>$ $6.5 \%$ ), what is not compatible with fractional crystallization.

2. $\mathrm{Al}_{2} \mathrm{O}_{3}, \mathrm{Na}_{2} \mathrm{O}, \mathrm{Sr}, \mathrm{TiO}_{2}$, and $\mathrm{V}$ are positively correlated with $\mathrm{Zr}$ and negatively correlated with $\mathrm{MgO}$ which indicates fractional crystallization of olivine and clinopyroxene without participation of $\mathrm{Fe}$ $\mathrm{Ti}$-oxide and major plagioclase fractionation; $\mathrm{Ti}$ and $\mathrm{V}$ increase and $\mathrm{Fe}$ decreases with increasing $\mathrm{Zr}$ (Figs. 9) precluding ilmenite or $\mathrm{Ti}$-magnetite fractionation, and favoring early $\mathrm{Fe}$ incorporation into the pyroxenes or mixing of different magma batches.

To explain the observed trends in element variations two slightly different magma batches are inferred which underwent the same fractional crystallization process:

1. One (late?) batch has a more primitive and incompatible element enriched, more alkalic composition with high $\mathrm{Al}, \mathrm{Sr}, \mathrm{Ti}, \mathrm{V}, \mathrm{Zr}$, and $\mathrm{Mg}$, and low $\mathrm{Fe}$ concentrations.

2. Another batch has a more tholeiitic, evolved composition with significantly lower $\mathrm{Al}$ etc., and higher Fe concentrations.

The REE patterns (Fig. 11) support the idea of two slightly different magmas. Two different slopes and crossing of the lines indicate two different batches, one being more enriched in incompatible and LREE elements than the other. The wide range in plagioclase compositions, distinct for each subset, also supports the existence of compositionally slightly different magma batches. Olivine, clinopyroxene, and very minor plagioclase (no visible negative Eu anomaly) fractionation led to the evolved basalt compositions, which have also been found by LeRoex et al. (1983) in fracture zones of the Southwest Indian Ridge.

\section{Source Region}

Normalization of the trace element budget of the three least altered basalts against N-MORB is used to further constrain possible source regions and contaminants (Fig. 16). The following information can be drawn from the trace element pattern (Fig. 16):

1. Ba and Th are strongly enriched with respect to the N-MORB normalizer.

2. The High-Field-Strength-Elements (HFSE) $\mathrm{Ta}, \mathrm{Nb}, \mathrm{Zr}, \mathrm{Hf}$, and $\mathrm{Ti}$ are only very slightly enriched, and a $\mathrm{Ta}$ and $\mathrm{Nb}$ trough is evident. $\mathrm{Cr}$ also seems to be depleted, although this may be caused by fractional crystallization.

3. The LREE, with Ce as indicator, are enriched; the HREE, with $\mathrm{Yb}$ as an indicator, are slightly depleted (compare Fig. 11).

4. $\mathrm{Sr}, \mathrm{Rb}$, and $\mathrm{K}$ are less enriched than the LREE, and especially $\mathrm{Ba}$ and Th. The slight enrichment in $\mathrm{K}$ and $\mathrm{Rb}$ against MORB may be due to fractionation processes. In other words, the low $\mathrm{K}$ and $\mathrm{Rb}$ concentrations bear N-MORB signature.

Significant upper crustal, granitic material to contaminate Site 738 can be excluded, as it should cause $\mathrm{SiO}_{2}, \mathrm{Sr}, \mathrm{K}$, and $\mathrm{Rb}$ enrichment in the fresh samples. When enriched, this is due to alteration processes as previously discussed.

Site 738 basalts partially resemble plume-component-bearing basalts from Iceland (Rison and Craig, 1983), Hawaii (e.g., Frey and Clague, 1983) and some Columbia River basalts (Brandon and Goles, 1988). The similarities include the $\mathrm{Ba}$, and partially the Th enrichment, the $\mathrm{Sr}, \mathrm{K}$, and $\mathrm{Rb}$ depletion, and the LREE enrichment. Smooth trace element patterns should be expected, if only a plume-derived, enriched component would be responsible for the enriched character of the three Site 738 basaltic samples. To thus explain the particular Ba, Th, and LREE enrichment, a complementary source has to be invoked that specifically contributes these elements and retains HFSE, especially $\mathrm{Nb}$ and $\mathrm{Ta}$. 


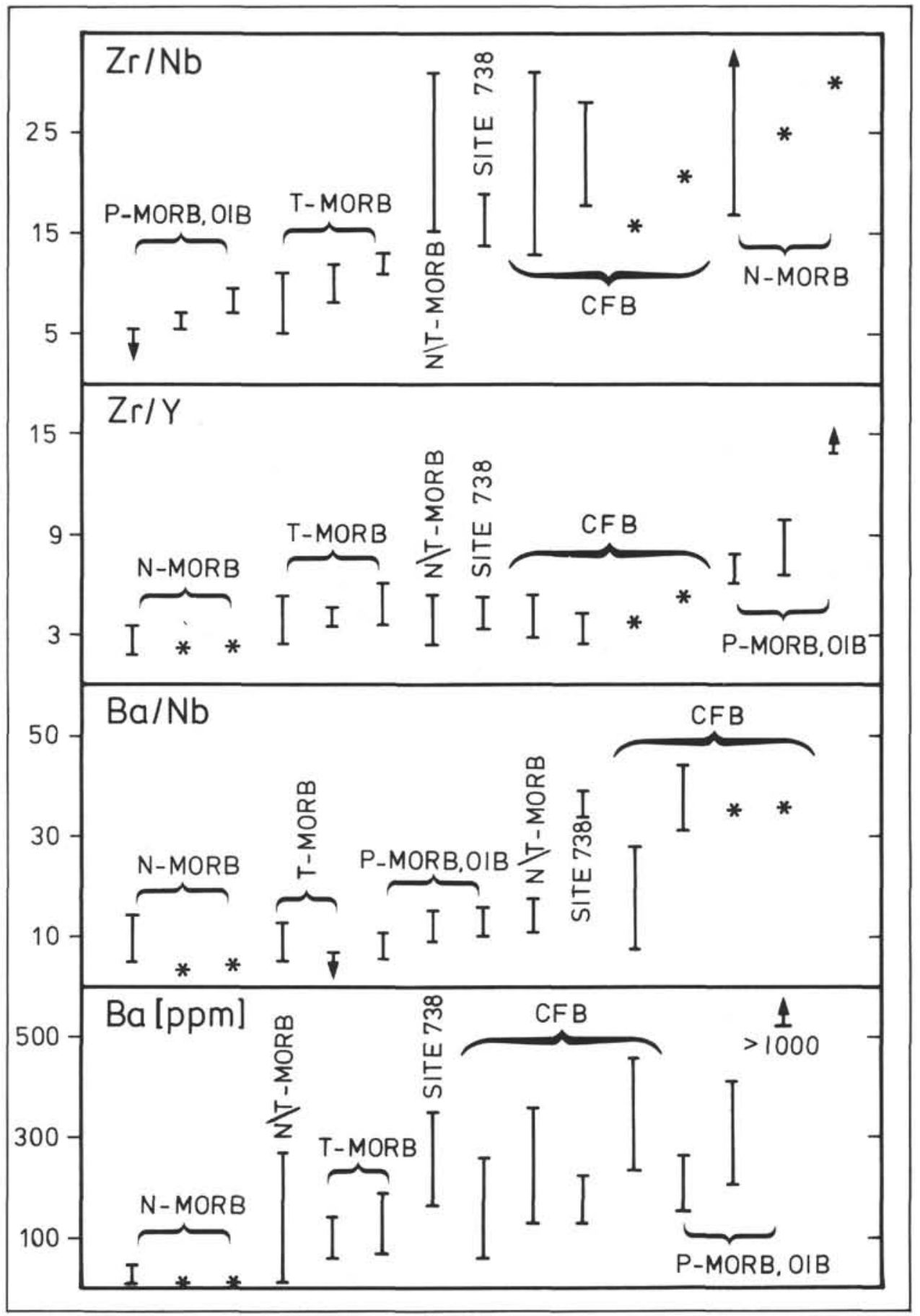

Figure 15. $\mathrm{Zr} / \mathrm{Nb}, \mathrm{Zr} / \mathrm{Y}, \mathrm{Ba} / \mathrm{Nb}$ ratios, and $\mathrm{Ba}$ concentrations of various oceanic basalts and continental basalts from southern hemisphere divergent plate boundaries, compared with Site 738 T-MORB. For references see Table 9.

Low $\mathrm{Ti} / \mathrm{Zr}$ ratios (64-73) and the high LREE/HREE ratios point to an enriched-mantle source, while the $\mathrm{Zr} / \mathrm{Y}$ ratios (3.3-5.3) and relatively low $\mathrm{Rb}, \mathrm{K}$, and $\mathrm{Sr}$ abundances (Fig. 16) may point to a less-enriched to depleted source component. Finally, a third source component is inferred to be responsible for the $\mathrm{Ba}$ and $\mathrm{Th}$ hump, the $\mathrm{Nb}$ and $\mathrm{Ta}$ trough, the high $\mathrm{Ba} / \mathrm{Nb}$ ratios, and the DUPAL isotope characteristics (Alibert, this volume).

High $\mathrm{Ba} / \mathrm{Ta}(>300), \mathrm{Th} / \mathrm{Ta}(>4)$, and low $\mathrm{Ta} / \mathrm{La}(<0.03)$ ratios are considered as evidence for contamination of continental flood basalts with some sort of crustal material (Loubet et al., 1988). Site 738 basalts have $\mathrm{Ba} / \mathrm{Ta}$ ratios around 480 , a Th/Ta ratio of 4 , and a $\mathrm{Ta} / \mathrm{La}$ ratio of 0.034 , which strongly point to crustal involvement. Further evidence for crustal derived contamination comes from the $\mathrm{Nb}$ and $\mathrm{Ta}$ troughs (Fig. 16), the small though recognizable $\mathrm{P}, \mathrm{Zr}, \mathrm{Hf}, \mathrm{Ti}$, and $\mathrm{Yb}$ depletion, and the $\mathrm{Ba}$ and Th bows, as emphasized by Thompson et al. (1984). This pattern is particularly characteristic for continental flood basalts (Thompson et al., 1984) and for subduction-related oceanic basalts (Wood et al., 1979; Pearce, 1982). Considering Table 2 and the high $\mathrm{Sr}_{i}$ ratios (Alibert, this volume), the striking similarity with continental flood basalts is evident. Continental flood basalts typically 
have high $\mathrm{Ba}$ and low $\mathrm{Nb}$ concentrations (Dupuy and Dostal, 1984), which similarly indicate subduction-related trace element patterns. Lightfoot and Hawkesworth (1988) emphasized the particular relationship between continental flood basalts and T-MORB. We therefore infer a source that contributes $\mathrm{Ba}$ and $\mathrm{Th}$ and retains the HFSE, especially $\mathrm{Nb}$ and Ta. These characteristics are typical for subduction-related arc basalts (Wood et al., 1979; Pearce 1982), the source of which retains HFSE as a result of refractory phases stable under high $\mathrm{pH}_{2} \mathrm{O}$ and $\mathrm{pO}_{2}$. This source is addressed as old, subducted, and partially remelted oceanic crust (Wood et al., 1979; Pearce, 1982; Hofmann and White, 1982; Thompson et al., 1984). After dehydration, partial melts from this "old" recycled oceanic crust, now a mantle section, still bear N-MORB signatures. When partial melts with MORB compositions from this deep reservoir rise, they vein the overlying mantle segment and interact with it. Interaction of rising basaltic magma with the overlying, thick mantle lherzolite may lead to HFSE and HREE depletion of the resulting magma due to incorporation of these elements into the refractory mantle mineral assemblage (olivine, orthopyroxene, spinel) with high crystal/liquid distribution coefficients (Kelemen et al. 1990). The resulting magma is depleted in HFSE, $\mathrm{Cr}, \mathrm{Ni}$, which are retained in the refractory mineral assemblage, and enriched in LREE and large ion lithophile elements (Kelemen et al. 1990), except for $\mathrm{K}$ and Rb, which may have escaped during former dehydration of the inferred downgoing slab.

It is concluded that the Site 738 basalts are derived from a veined upper mantle as proposed for the origin of T-MORB from the Southwest Indian Ridge (LeRoex et al., 1983). This veined upper mantle developed from interaction of basaltic liquids, derived from dehydrated, recycled oceanic crust underneath, with an overlying lherzolitic upper mantle. Slight differences in the degree of partial melting, and heterogeneity of the source are responsible for the observed slightly different magma batches. Ba and Th bows, LREE enrichment, HREE depletion, and $\mathrm{Nb}$ and Ta troughs (Fig. 16) can be explained by contamination of the source region with partial melts derived from dehydrated subducted oceanic(?) crust. This model needs thermal energy from a rising thermal plume (generation of large volumes of basaltic melts), which is the Kerguelen-Heard plume/hot spot. It does not need any continental crust material, nor a specific P-MORB/OIB magma.

The following model for the source region of Site 738 basalts is adopted from the above considerations:

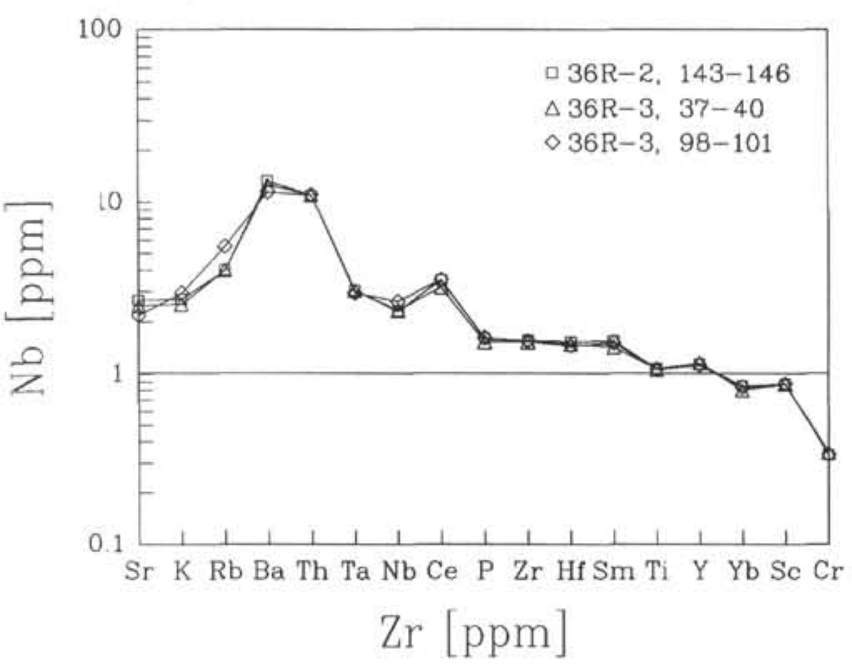

Figure 16. N-MORB-normalized element patterns of fresh Hole 738C samples. Normalization values from Pearce (1982).
1. Hot spot/plume-related heat transfer to the lithosphere is made responsible for the large amounts of partial melts (i.e., Kerguelen Plateau basalts).

2. Interaction of rising basaltic liquids with overlying, thickened(?), lherzolitic upper mantle creates a veined upper mantle. Partial melting of this veined upper mantle is responsible for low HREE, $\mathrm{Cr}(?), \mathrm{Nb}$, and $\mathrm{Ta}$ concentrations (stored in refractory upper mantle mineral assemblage), high LREE, Ba, and Th concentrations (from the recycled basaltic crust wedge), and high $\mathrm{Sr}$ and $\mathrm{Pb}$ isotope values (cf. Alibert, this volume) of Site 738 basalts.

This model partially follows the suggestions from Hofmann and White (1982), where oceanic crust is subducted into the mantle, sinks to the lower mantle boundary, becomes heated, dehydrated, and partially melted. These partial melts have N/T-MORB(?) basaltic composition, migrate upward and vein the overlying lithosphere. The model also takes into account the suggestions of Kelemen et al. (1990), where basaltic liquid-upper mantle interaction is made responsible for HFSE and HREE depletions and LILE and LREE enrichments of arc-basaltic magma.

\section{CONCLUSIONS}

1. The basaltic rocks of Site 738 of ODP Leg 119 belong to the plateau-forming stage (pre-Turonian) of southern Kerguelen Plateau formation. The aa- lava flows with basal and top breccias erupted under subaerial to shallow water, highly oxidizing conditions.

2. The basement rocks are tholeiitic T-MORB with $\mathrm{Zr} / \mathrm{Nb}$ around 17 and LREE enrichment, and high $\mathrm{Ba}$ and $\mathrm{Th}$, and low $\mathrm{Ta}$ and $\mathrm{Nb}$ concentrations. CIPW norms, clinopyroxene phenocryst compositions (Ca-poor and $\mathrm{Ca}$-rich), and plagioclase compositions (linear orthoclase increase) confirm the tholeiitic and transitional character of the basalts.

3. The Site 738 T-MORB have undergone significant olivine (estimated about $8 \%$ ) and some clinopyroxene fractionation (low $\mathrm{Ni}$, medium $\mathrm{Cr}$, and $\mathrm{MgO}$ concentrations) prior to eruption. Mixing between a more primitive, $\mathrm{Mg}$-, $\mathrm{Al}-, \mathrm{Ti}-$, and $\mathrm{Zr}$-rich, Fe-poor, slightly alkalic T-MORB-type magma, and a more evolved T/N-MORB-type tholeiitic magma with lower $\mathrm{MgO}, \mathrm{Al}_{2} \mathrm{O}_{3}, \mathrm{TiO}_{2}$, and $\mathrm{Zr}$ and higher $\mathrm{FeO}$ can account for the observed variations in major and trace elements, and the crossing REE patterns.

4. The source region is an enriched, veined upper mantle, bearing refractory phases which retain the HFSE, HREE, and especially $\mathrm{Nb}$ and $\mathrm{Ta}$.

5. Partial melts from an old, subducted, and recycled, oceanic(?) crustal component are the liquids veining the upper mantle, and are made responsible for the $\mathrm{Ba}$ and Th enrichment.

6. The secondary mineral assemblage includes saponite, celadonite, montmorillonite (?), and $\mathrm{Fe}$-oxides and $\mathrm{Fe}$-hydroxides indicative of low-temperature seawater alteration $\left(<170^{\circ} \mathrm{C}\right)$. Precipitation of calcite is due to terminal cold-seawater alteration.

7. The alteration caused extensive oxidation and net gains in $\mathrm{H}_{2} \mathrm{O}$, $\mathrm{CO}_{2}, \mathrm{~K}_{2} \mathrm{O}$, and $\mathrm{Rb}$ while $\mathrm{CaO}$ and $\mathrm{V}$ were released.

\section{ACKNOWLEDGMENTS}

Research was funded by DFG (German Science Foundation) grants Schm 250/37-2/3 to H.-U. Schmincke. H. Niephaus, B. Schulz, and H.-J. Bernhard, all from Bochum University, helped with XRF and microprobe work. J. Dehn assisted with computer skills and B. Lehmann with drafting. To all of them we express our gratitude. Critical reviews by two anonymous reviewers helped to clarify results and are gratefully acknowledged. 


\section{REFERENCES}

Andrews, A. J., 1979. On the effect of low-temperature seawater-basalt interaction on the distribution of sulfur in oceanic crust, Layer 2. Earth Planet. Sci. Lett., 46:68-80.

1980. Saponite and celadonite in layer 2 basalts, DSDP Leg 37. Contrib. Mineral. Petrol., 73:323-340.

Andrews, A. J., Bamett, R. L., MacClement, B.A.E., Fyfe, W. S., Morrison, G., MacRae, N. D., and Starkey, J., 1977. Zeolite facies metamorphism, geochemistry and some aspects of trace element redistribution in altered basalts of DSDP, Leg 37. In Aumento, F., Melson, W. G., et al., Init. Repts. DSDP, 37: Washington (U.S. Govt. Printing Office), 795-810.

Baker, I., and Haggerty, S. E., 1967. The alteration of olivine in basaltic and Associated lavas. Part II: intermediate and low temperature alteration. Contrib. Mineral. Petrol., 16:258-272.

Barron, J., Larsen, B., et al., 1989. Proc. ODP, Init. Repts., 119: College Station, TX (Ocean Drilling Program).

Bassias, Y., Davies, H., Leclaire, L., and Weis, D., 1987. Basaltic basement and sedimentary rocks from the southern sector of the Kerguelen-Heard Plateau: new data and their Meso-Cenozoic paleogeographic and geodynamic implication. Bull. Mus. Natl. Hist. Nat., Sect. C, 9:367-403.

Bednarz, U., and Schmincke, H.-U., 1989. Mass transfer during sub-seafloor alteration of the upper Troodos crust (Cyprus). Contrib. Mineral. Petrol. 102:93-101.

Bitschene, P. R., 1987. Mesozoischer und känozoischer, anorogener Magmatismus in Ostparaguay: Arbeiten zur Geologie und Petrologie zweier Alkaliprovinzen [Ph.D. dissert.]. Ruprecht-Karls-Univ., Heidelberg, FRG.

Bitschene, P. R., Dehn, J., Mathis, J., Mehl, K., and Schmincke, H.-U., 1989. Die vulkano-tektonische Entwicklung im Indischen Ozean (Kerguelen Plateau, Broken Ridge, Ninetyeast Ridge): Ergebnisse der ODP Legs 119, 120 und 121. Nachr. Dtsch. Geol. Ges., 41:12-13. (Abstract)

Bitschene, P. R., Mehl, K. W., and Schmincke, H.-U., in press. Tephra layers from the Kerguelen Plateau, southem Indian Ocean (ODP Legs 119 and 120). In Schlich, R., Wise, S. W., Jr., et al., Proc. ODP, Sci. Results, 120: College Station, TX (Ocean Drilling Program).

Brandon, A. D., and Goles, G. G., 1988. A Miocene subcontinental plume in the Pacific Northwest: geochemical evidence. Earth Planet. Sci. Lett., $88: 273-283$.

Davies, H. L., Sun, S.-S., Frey, F. A., Gautier, I., McCulloch, M. T., Price, R. C., Bassias, Y., Klootwijk, C. T., and Leclaire, L., 1990. Basalt basement from the Kerguelen Plateau and the trail of the Dupal plume. Contrib. Mineral. Petrol., 103:457-469.

Deer, W. A., Howie, R. A., and Zussman, J., 1978. An Introduction to the Rock Forming Minerals: New York (Longman).

Donnelly, T. W., Thompson, G., and Salisbury, M. H., 1980. The chemistry of altered basalts at Site 417, DSDP Leg 51. In Donnelly, T., Francheteau, J., Bryan, W., Robinson, P., Flower, M., Salisbury, M., et al., Init. Repts. , 51, 52,53 (Pt. 2): Washington (U.S. Govt. Printing Office), 1319-1330.

Dosso, L., Bougault, H., Beuzart, P., Calvez, J.-Y., and Joron, J.-L., 1988. The geochemical structure of the South East Indian Ridge. Earth Planet. Sci. Lett., 88:47-49.

Duncan, A. R., Erlank, A. J., and Marsh, J. S., 1984. Regional geochemistry of the Karroo igneous province. In Erlank, A. J. (Ed.) Petrogenesis of the Volcanic Rocks of the Karoo Province. Spec. Publ. Geol. Soc. S. Afr., 13:355-388.

Dupuy, C., and Dostal, J., 1984. Trace element geochemistry of some continental tholeiites. Earth Planet. Sci. Lett. 67:61-69.

Erlank, A. J., 1984. Petrogenesis of the volcanic rocks of the Karroo Province. Geol. Soc. S. Afr. Spec. Publ., 13, 1-395.

Frey,F.A., and Clague, D. A., 1983. Geochemistry of diverse basalt types from Loihi seamount, Hawaii: petrogenetic implications. Earth Planet. Sci.Lett., 66:337-355,

Gijbels, R., 1980. Reconstruction of the trace element distribution in the late Quaternary differentiated magma chambers of the Laacher See volcano (East Eifel). Final Rep., EC contract 219-77 EGB, Univ. of Antwerpen: $1-60$.

Hart, S. R., 1984. A large scale isotope anomaly in the Southern Hemisphere mantle. Nature, 309:753-757.

Hertogen, J., and Gijbels, R., 1981. Instrumental neutron activation analysis of silicate rocks with low-energy photon detector. Geochim. Cosmochim. Acta, 56:61-82.

Hofmann, A. W., and White, W. M., 1982. Mantle plumes from ancient oceanic crust. Earth Planet. Sci. Lett., 27:421-436.
Kelemen, P. B., Johnson, K.T.M., Kinzler, R. J., and Irving, A. J., 1990. High-field-strength element depletion in arc basalts due to mantle-magma interaction. Nature, 345:521-524.

Klein, E. M., and Langmuir, C. H., 1988. Ocean ridge basalt chemistry, axial depth, crustal thickness and temperature variations in the mantle. J. Geophys. Res., 92:8089-8115.

Lawrence, J. R., 1980. Temperatures of formation of calcite veins in the basalts from Deep Sea Drilling Project Holes 417A and 417D. In Donnelly, T. Francheteau, J., et al., Init. Repts. DSDP, 51, 52, 53 (Pt. 2): Washington (U.S. Govt. Printing Office), 1183-1184.

LeRoex, A. P., 1987. Source regions of mid-ocean ridge basalts: evidence for enrichment processes. In Menzies, M. A., and Hawkesworth, C. J. (Eds.), Mantle Metasomatism: London (Academic Press), 389-422.

LeRoex A. P., Dick H.J.B., Erlank A. J., Reid A. M., Frey F. A., and Hart, S. R., 1983. Geochemistry, mineralogy and petrogenesis of lavas erupted along the Southwest Indian Ridge between the Bouvet Triple Junction and 11 degrees east. J. Petrol., 24:267-318.

Lightfoot, P., and Hawkesworth, C., 1988. Origin of Deccan Trap lavas: evidence from combined trace element and $\mathrm{Sr}-, \mathrm{Nd}$ - and $\mathrm{Pb}$-isotope studies. Earth Planet. Sci. Lett., 91:89-104.

Loubet, M., Sassi, R., and Di Donato, G., 1988. Mantle heterogeneities: a combined isotope and trace element approach and evidence for recycled continental crust materials in some OIB sources. Earth Planet. Sci. Lett., 89:299-315.

MacDonald, G. A., and Katsura, T., 1964. Chemical composition of the volcanic rocks of Hawaiian lavas. J. Petrol., 5: 82-133

Mahoney, J. J., Macdougall, J. D., Lugmair, G. W., and Gopalan, K., 1983. Kerguelen hotspot source for Rajmahal Traps and Ninetyeast Ridge? Nature, 303:385-389.

Manson, B., and Moore, C. B., 1982. Principles of Geochemistry (4th ed.): New York (Wiley).

Mantovani, M.S.M., Peate, D. W., and Hawkesworth, C. J., 1988. Geochemical stratigraphy of Parana continental flood basalts: a contribution from borehole samples. In Piccirillo, E. M., and Melfi, A. J. (Eds.), The Mesozoic Flood Volcanism of the Parana Basin: Sao Paulo (Inst. Astronom. e Geofis. Publ.), 15-24.

Mehegan, J. M., and Robinson, P. T., 1982. Secondary mineralization and hydrothermal alteration in the Reydarfjordur Drill Core, Eastern Iceland. J. Geophys. Res., 87:6511-6524.

Mottl, M. J., and Holland, H. D., 1978. Chemical exchange during hydrothermal alteration of basalt by seawater. I. Experimental results for major and minor components of seawater. Geochim. Cosmochim. Acta, 42:1103-1115.

Pearce, J. A., 1982. Trace elements characteristics of lavas from destructive plate boundaries. In Thorpe, R. S. (Ed.), Andesites: Orogenic Andesites and Related Rocks: New York (Wiley), 525-548.

Rison, W., and Craig, H., 1983. Helium isotopes and mantle volatiles in Loihi seamount and Hawaiian island basalts and xenoliths. Earth Planet. Sci. Lett., 66:407-426.

Robinson, P. T., Flower, M.F.J., Schmincke, H.-U., and Ohnmacht, W., 1977. Low temperature alteration of oceanic basalts, DSDP Leg 37. In Aumento, F., Melson, W. G., et al., Init. Repts. DSDP, 37: Washington (U.S. Govt. Printing Office), 775-793.

Schlich, R., Wise, S. W., Jr., et al., 1989. Proc. ODP, Init. Repts., 120: College Station, TX (Ocean Drilling Program).

Seyfried, W.E., Jr., and Bischoff, J. L., 1979. Low temperature basalt alteration by seawater: an experimental study at $70^{\circ} \mathrm{C}$ and $150^{\circ} \mathrm{C}$. Geochim. Cosmochim. Acta, 43:1937-1947.

Seyfried, W. E., Jr., Shanks, W. C., III, and Dibble, W. E., Jr., 1978. Clay mineral formation in DSDP Led 34 basalt. Earth Planet. Sci. Lett., $41: 265-276$

Staudigel, H., and Hart, S. R., 1983. Alteration of basaltic glass: mechanisms and significance of the oceanic crust-seawater budget. Geochim. Cosmochim. Acta, 47:337-350.

Staudigel, H., Hart, S. R., and Richardson, S.H., 1981. Alteration of the oceanic crust: processes and timing. Earth Planet. Sci. Lett., 62:311-327.

Storey, M., Saunders, A. D., Tarney, J., Leat P., Thirlwall, M. F., Thompson, R. N., Menzies, M. A., and Marriner, G. F., 1988. Geochemical evidence for plume-mantle interactions beneath Kerguelen and Heard islands, Indian Ocean. Nature, 336:371-374.

Sun, S.-S., and McDonough, W. F., 1989. Chemical and isotopic systematics of oceanic basalts: implications for mantle composition and processes. In Saunders, A. D., and Norry, M. J., (Eds.), Magmatism in the Ocean Basins. Geol. Soc. Spec. Publ. London, 42:313-345. 
Sun, S.-S., Nesbitt, R. W., and Sharaskin, A. Y., 1979. Geochemical characteristics of mid-ocean ridge basalts. Earth Planet. Sci. Lett., 44:119-138.

Thompson, R. N., Morrison, M. A., Hendry, G. L., and Parry, S. J., 1984. An assessment of the relative roles of crust and mantle in magma genesis: an elemental approach. Philos. Trans. R. Soc. London, 310:549-590.

Viereck, L. G., Flower, M.F.J., Hertogen, J., Schmincke, H.-U., and Jenner, G. A., 1989. The genesis and significance of N-MORB sub-types. Contrib. Mineral. Petrol., 102:112-126.

Viereck, L. G., Griffin, B. J., Schmincke, H.-U., and Pritchard, R. G., 1982. Volcaniclastic rocks of the Reydarfjordur Drill Hole, eastern Iceland. 2. Alteration. J. Geophys. Res., 87:6459-6476.

Weaver, C. E., and Pollard, L. D., 1973. The Chemistry of Clay Minerals. Developments in Sedimentology, 15: Amsterdam (Elsevier).
Weis, D., Bassias, Y., Gautier, I., and Mennesier, J.-P., 1989. DUPAL anomaly in existence $115 \mathrm{Ma}$ ago: evidence from isotopic study of the Kerguelen Plateau (South Indian Ocean). Geochim. Cosmochim. Acta, 53:2125-2131.

Wood, D. A., Joron, J. L., Treuil, M., Norry, M. J., and Tarney, J., 1979. Elemental and $\mathrm{Sr}$ isotope variations in basic lavas from Iceland and the surrounding ocean floor. Contrib. Mineral. Petrol., 70:319-339.

Date of initial receipt: 2 January 1990

Date of acceptance: 7 August 1990

Ms 119B-130 

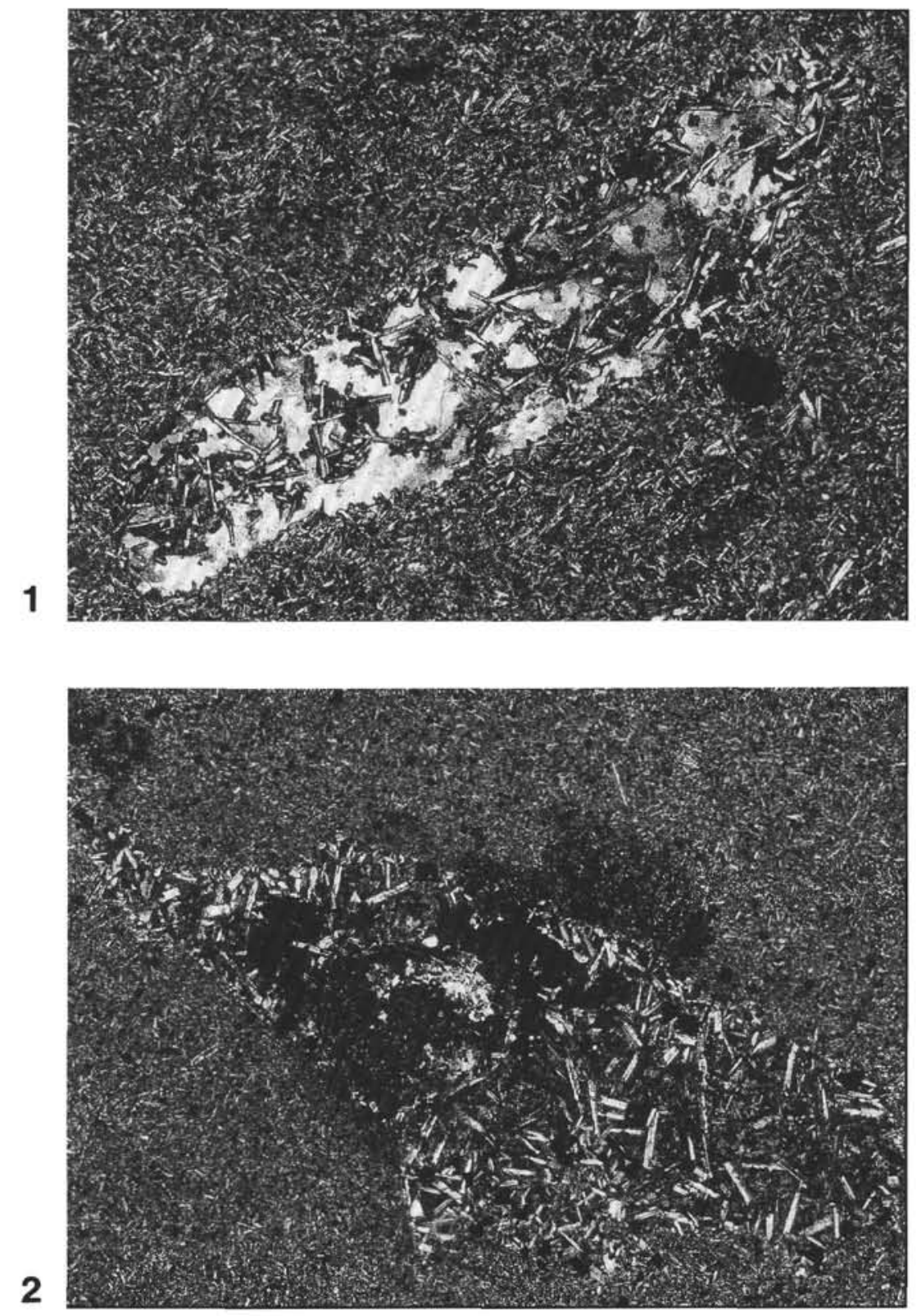

Plate 1. Pegmatoid "schlieren" in basalt. 1. Coarse plagioclase laths and clinopyroxene are rimmed by brown sheet silicates. Glassy matrix is replaced by calcite. Sample 119-738C-34R-4, 39-42 cm. Width of photo about $6 \mathrm{~mm}$. Crossed nicols. 2. Coarse plagioclase laths and clinopyroxene lie in a groundmass replaced by $\mathrm{K}$-feldspar. A vesicle (left of center) is filled by a dark brown sheet silicate, celadonite, and calcite. Sample 119-738C-33R-5, 30-32 cm. Width of photo about 9 $\mathrm{mm}$. Crossed nicols. 